\title{
Mechanistic Studies of Bismuth(V)-Mediated Thioglycoside Activation Reveal Differential Reactivity of Anomers
}

Manibarsha Goswami, ${ }^{1}$ Daniel C. Ashley, ${ }^{2}$ Mu-Hyun Baik ${ }^{2,3,4^{*}}$ and Nicola. L. B. Pohl ${ }^{2,5^{*}}$

${ }^{1}$ Department of Chemistry, lowa State University, Ames, IA 50011

${ }^{2}$ Department of Chemistry, Indiana University, Bloomington, IN 47405

${ }^{3}$ Department of Chemistry, Korea Advanced Institute of Science and Technology (KAIST), Daejeon 305-701, Korea

${ }^{4}$ Center for Catalytic Hydrocarbon Functionalizations, Institute for Basic Science (IBS), Daejeon 305-701, Korea

${ }^{5}$ Department of Chemical and Biological Engineering, lowa State University, Ames, IA 50011

Table of contents

GC-MS spectra

S2

NMR spectra.

S4

General Computational Methodology.

S18

Calculated Energies.

S21

DFT Methodology Benchmarking...

Cartesian Coordinates of Optimized Structures

S27

Vibrational Frequencies of Optimized Structures

S63

References. 


\section{GC spectra}

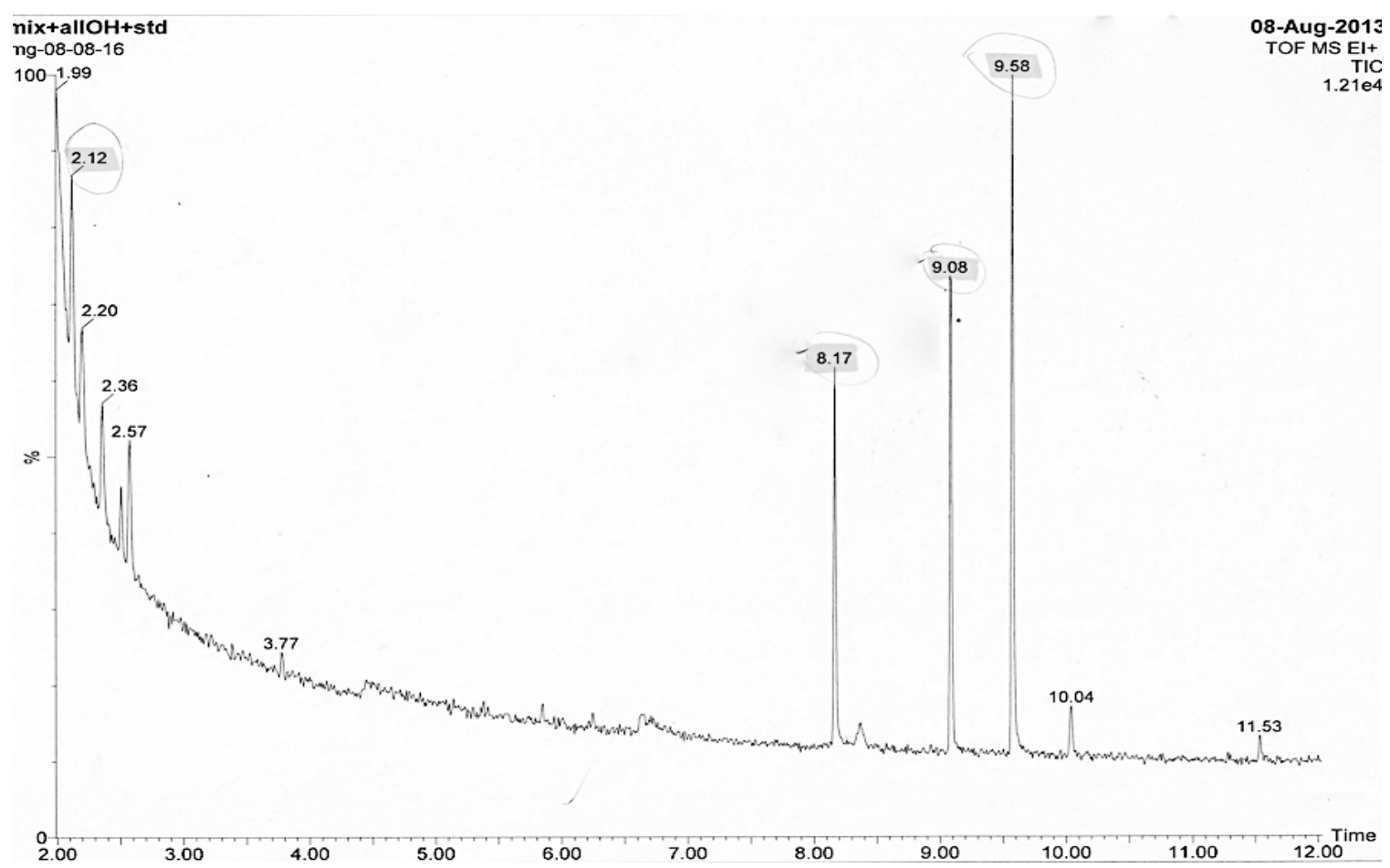

Analyte mixture containing $\mathrm{PrSH}, \mathrm{PhSPr}, \mathrm{PrSSPr}$ and dodecane

\section{Reaction monitoring}

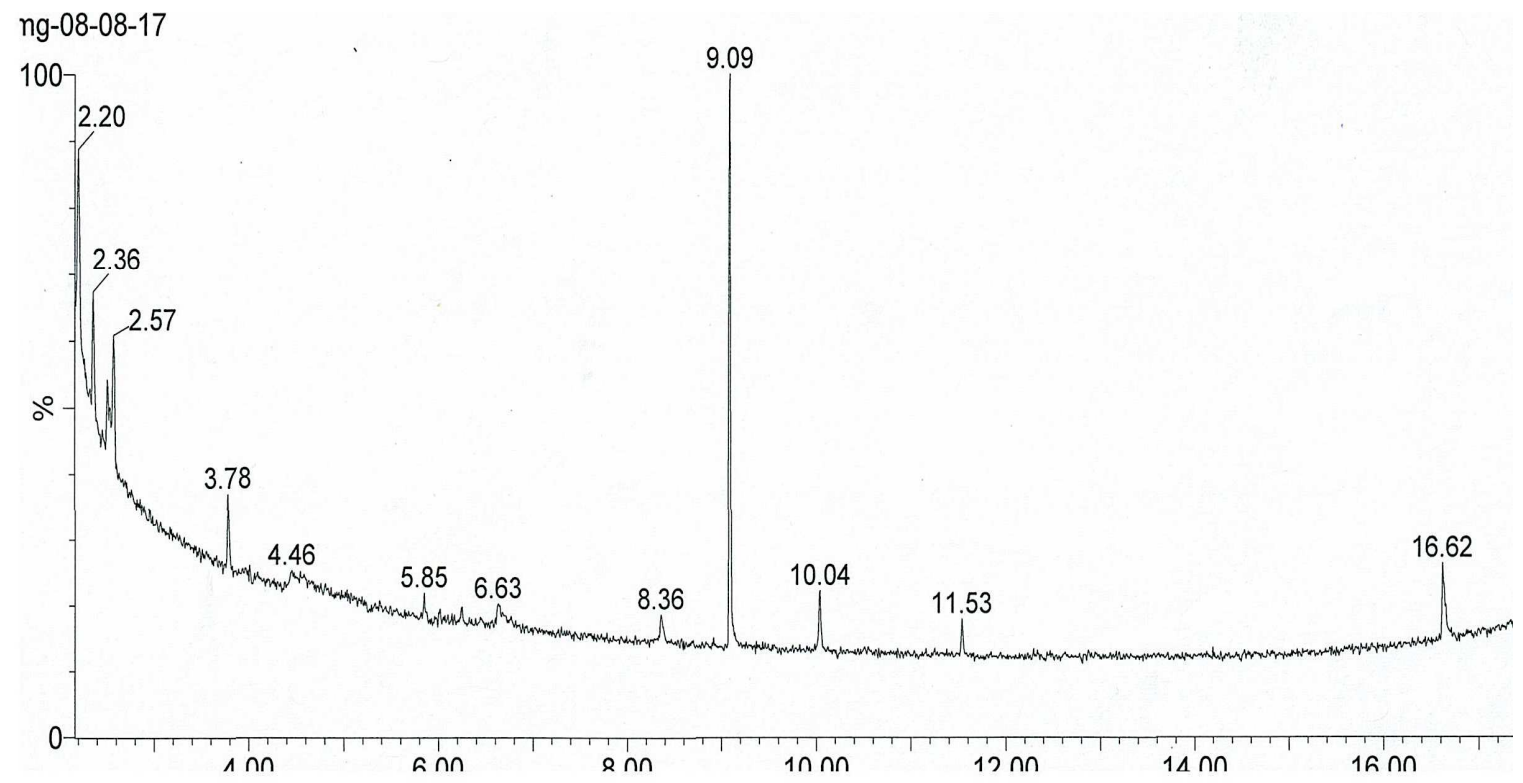

Reaction mixture after $\mathrm{t}=1 \mathrm{~min}$ 


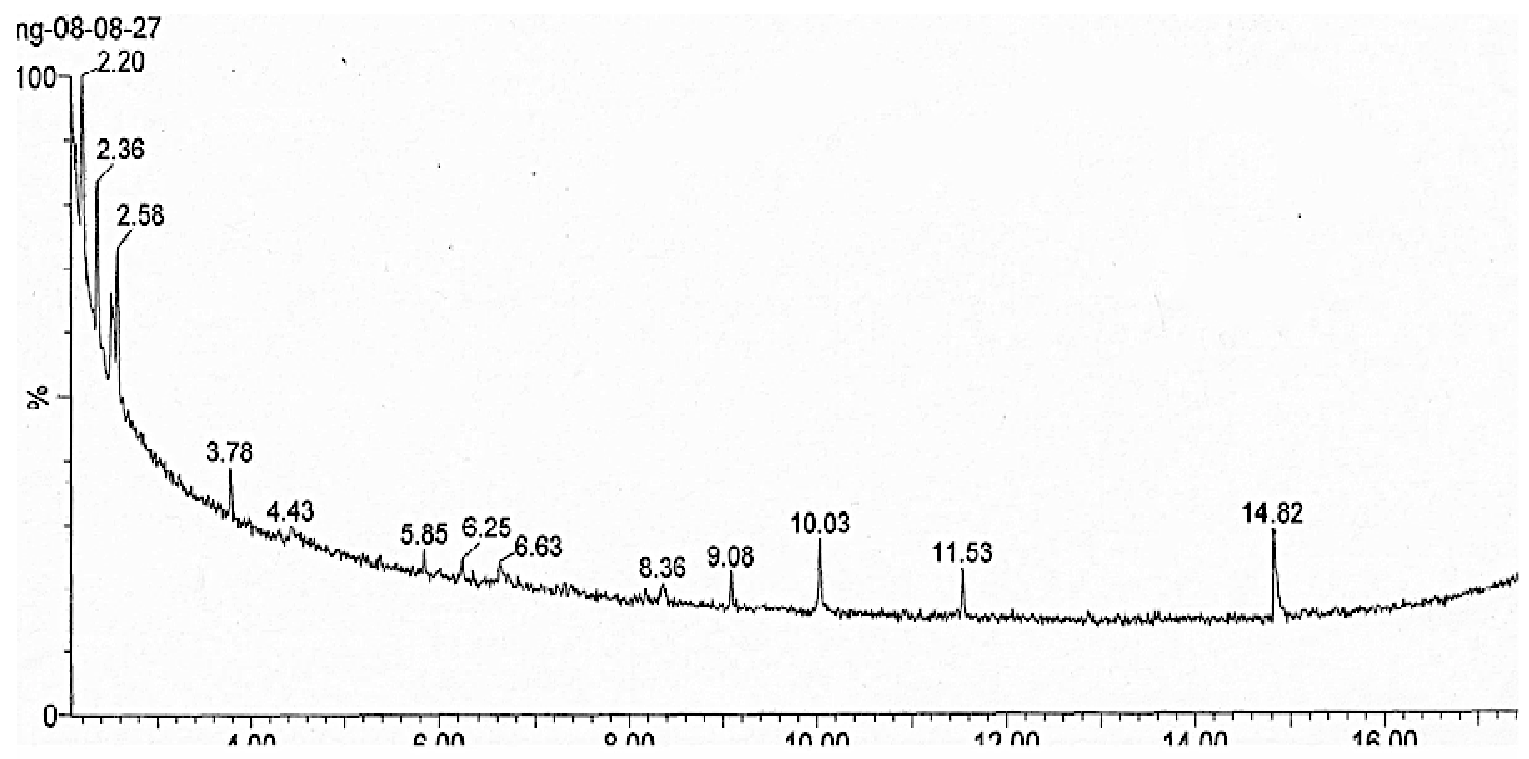

Reaction mixture after $\mathrm{t}=40 \mathrm{~min}$

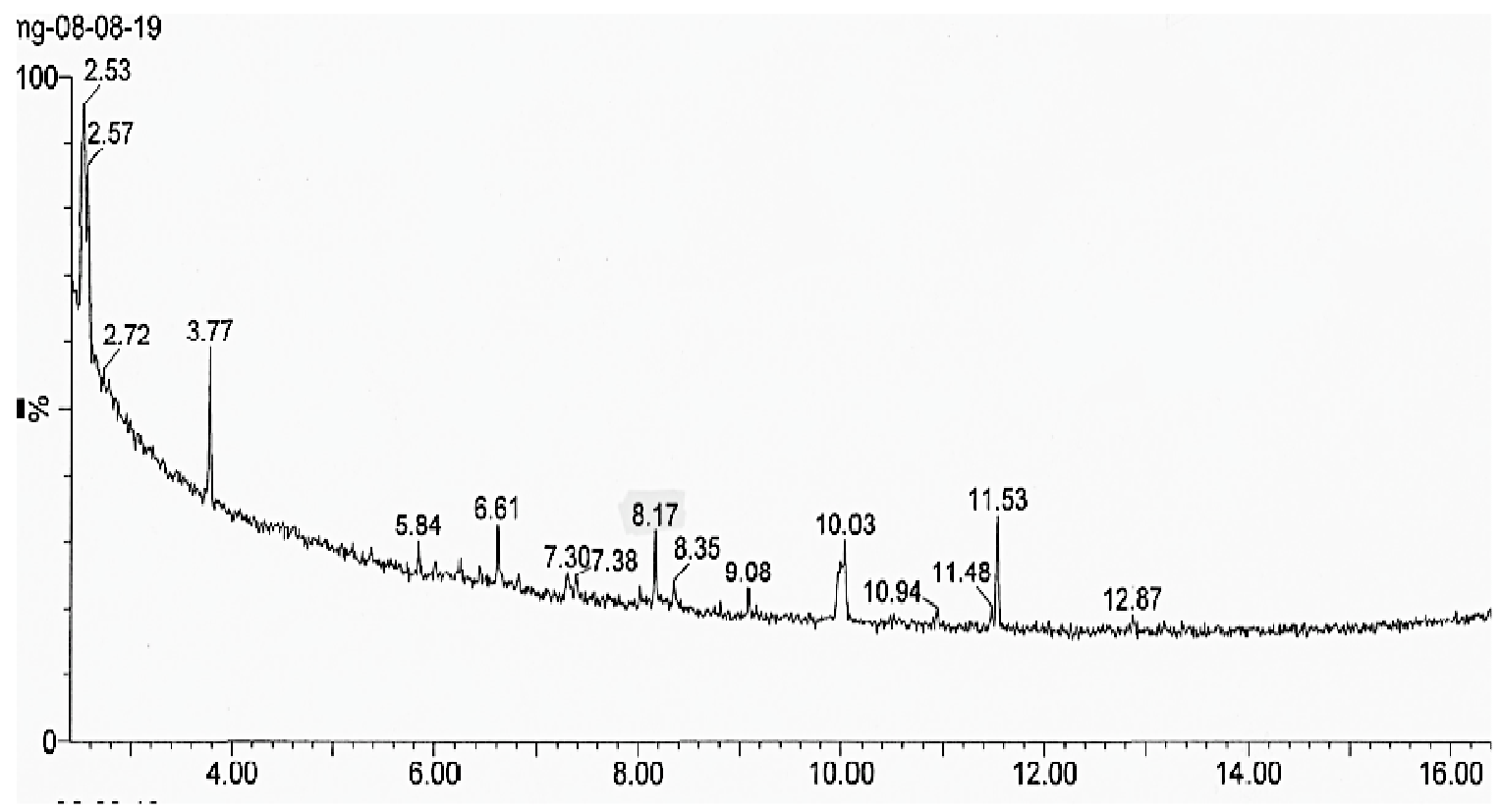

Reaction mixture after $\mathrm{t}=60 \mathrm{~min}$ 
NMR spectra

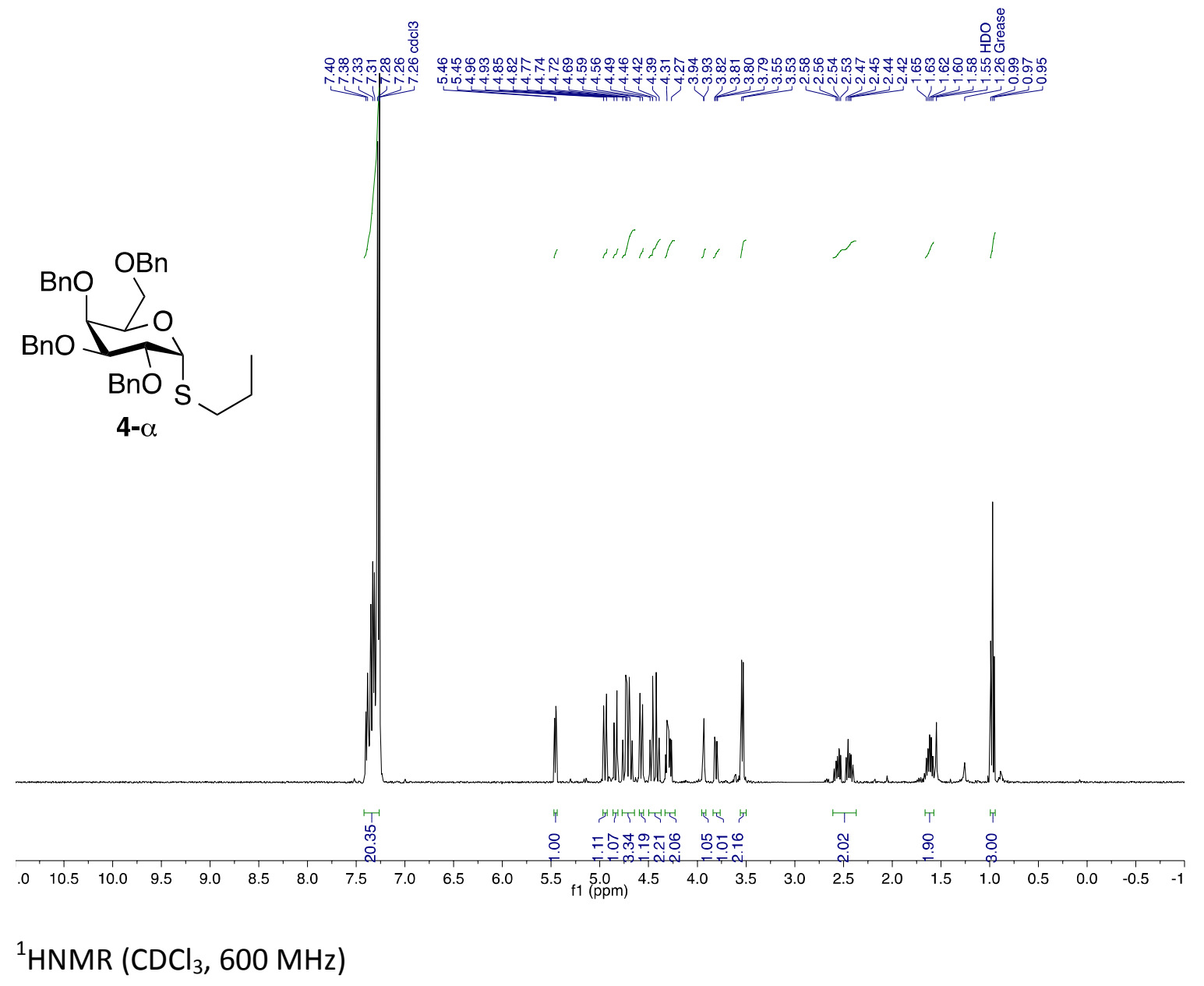



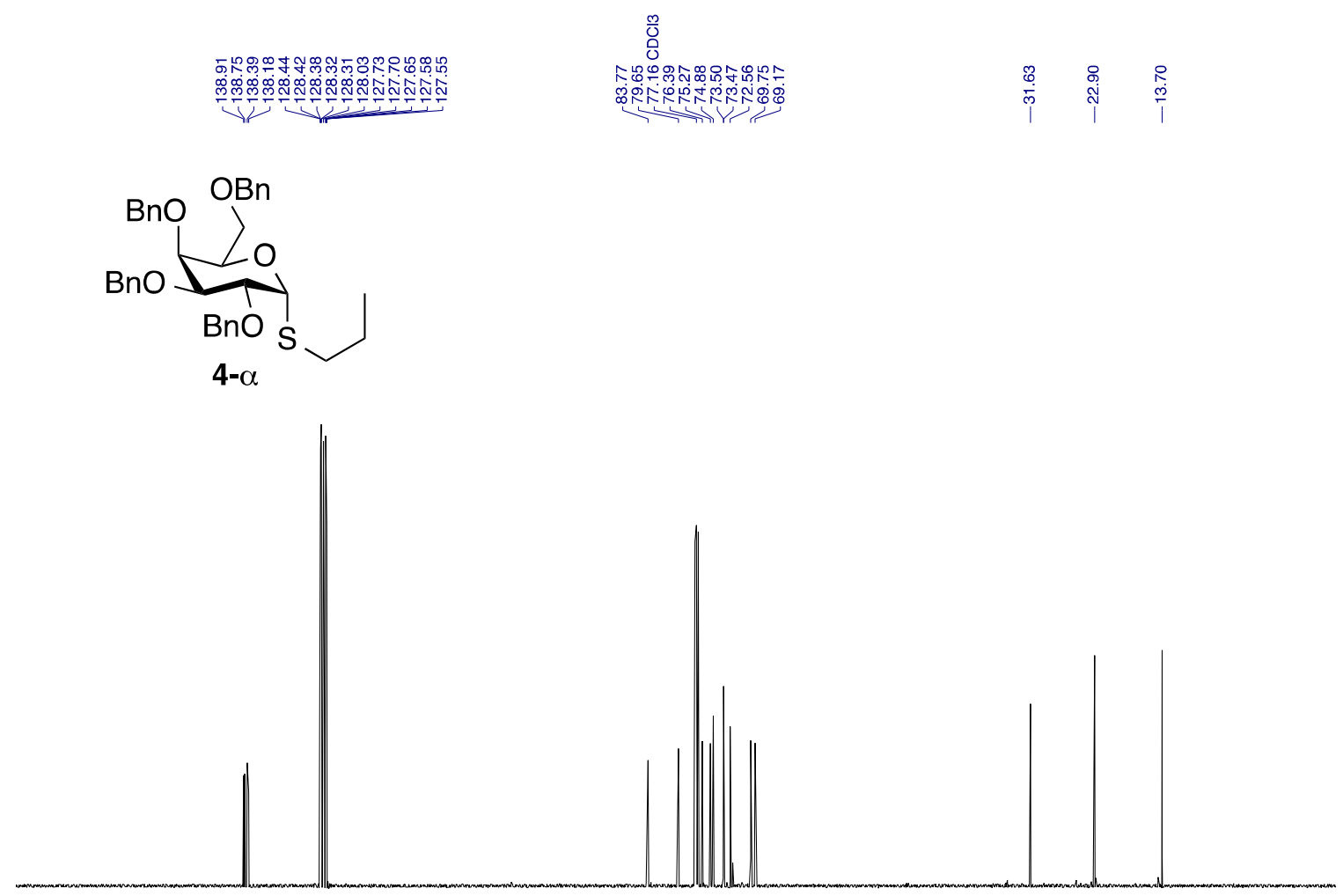

$160 \quad 150$
140

$\begin{array}{rrrr}130 & 120 & 110 & 100\end{array}$

$90 \quad 80$

$70 \cdot 60$

${ }^{13} \mathrm{CNMR}\left(\mathrm{CDCl}_{3}, 151 \mathrm{MHz}\right)$ 


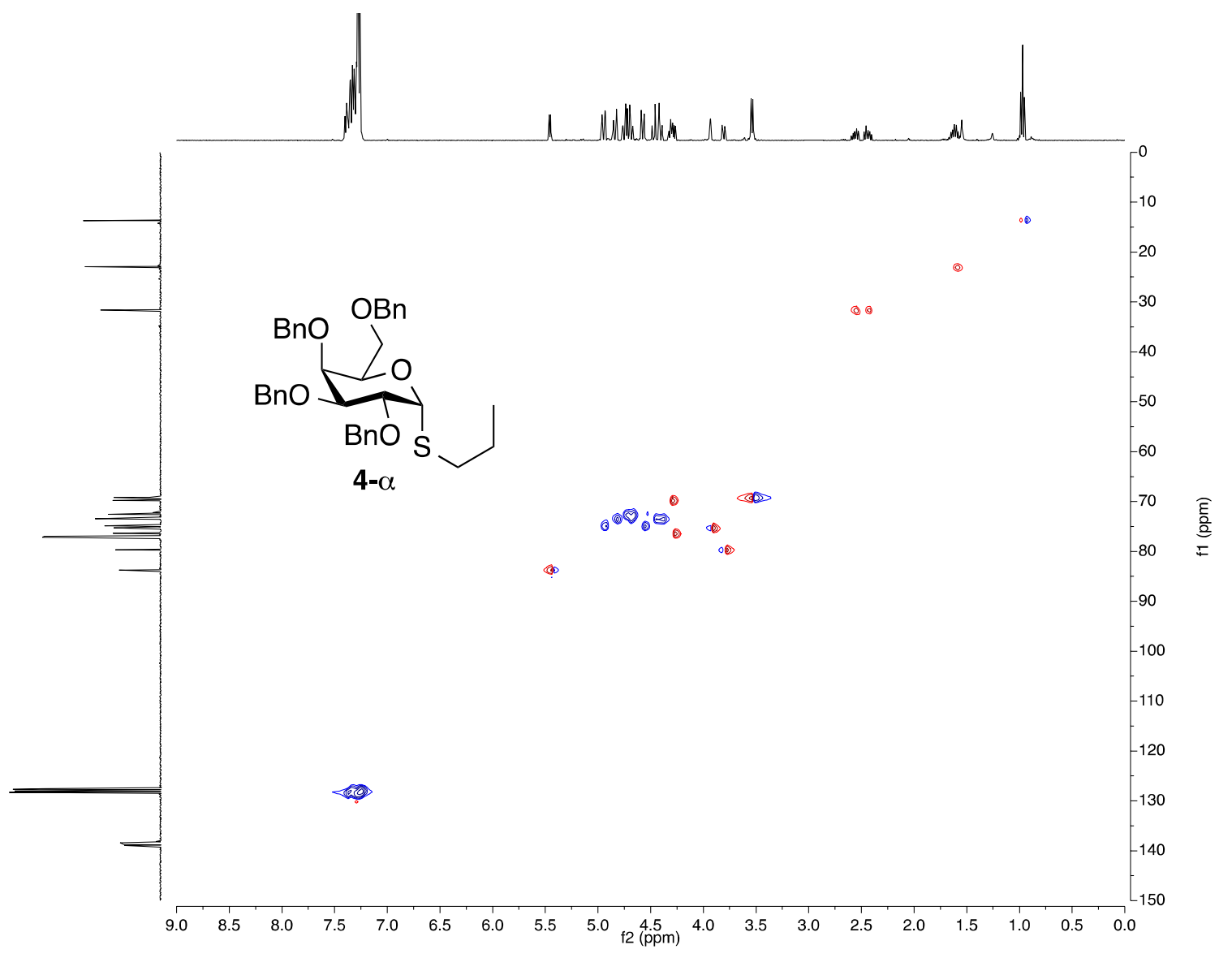

HSQC phase edit $\left(\mathrm{CDCl}_{3}\right)$ 

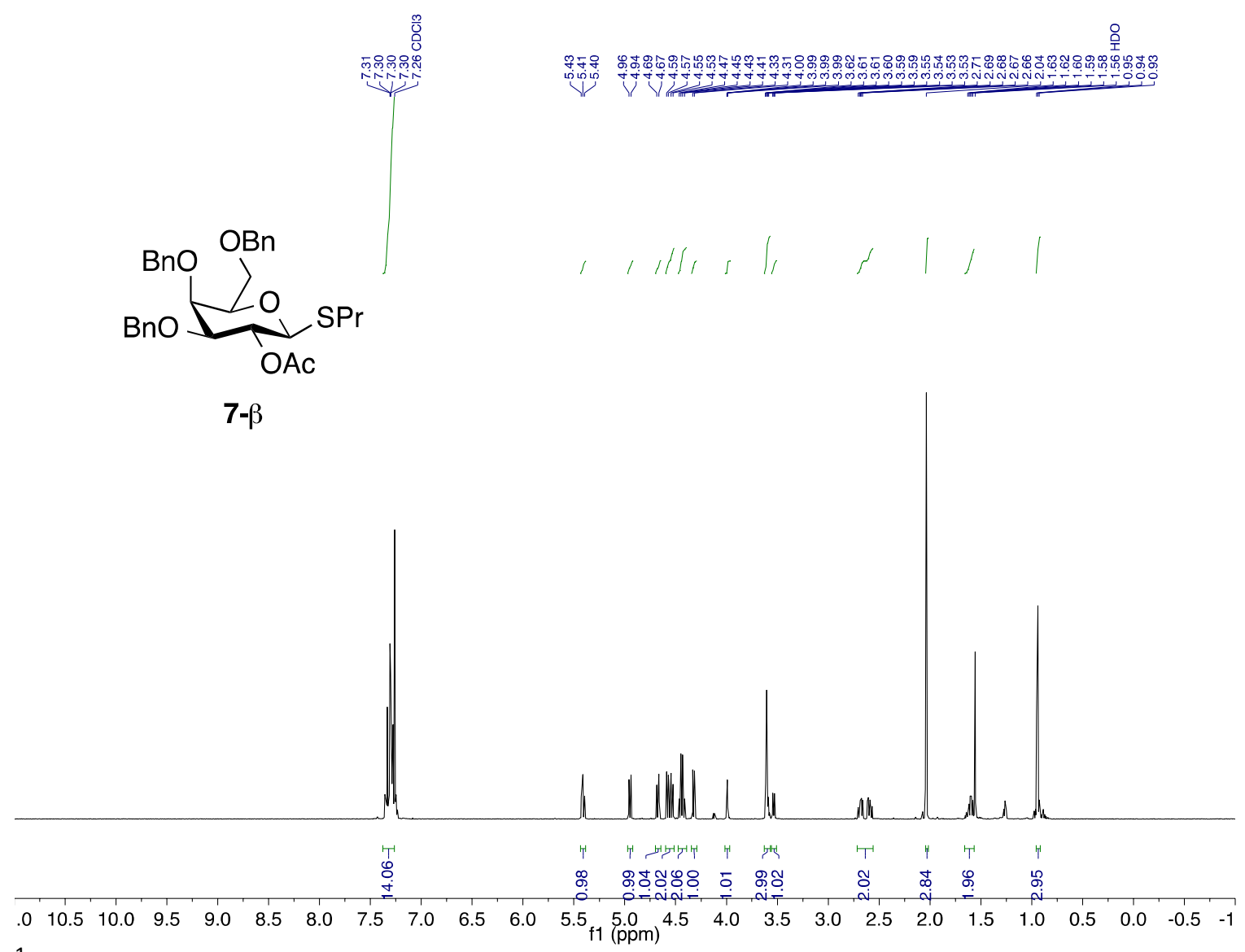

${ }^{1} \mathrm{HNMR}\left(\mathrm{CDCl}_{3}, 600 \mathrm{MHz}\right)$ 

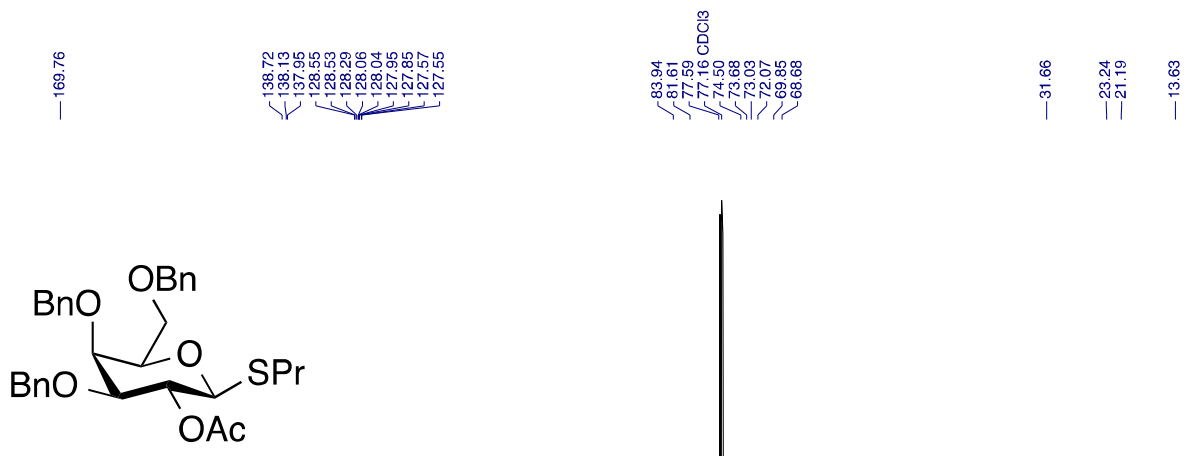

7- $\beta$

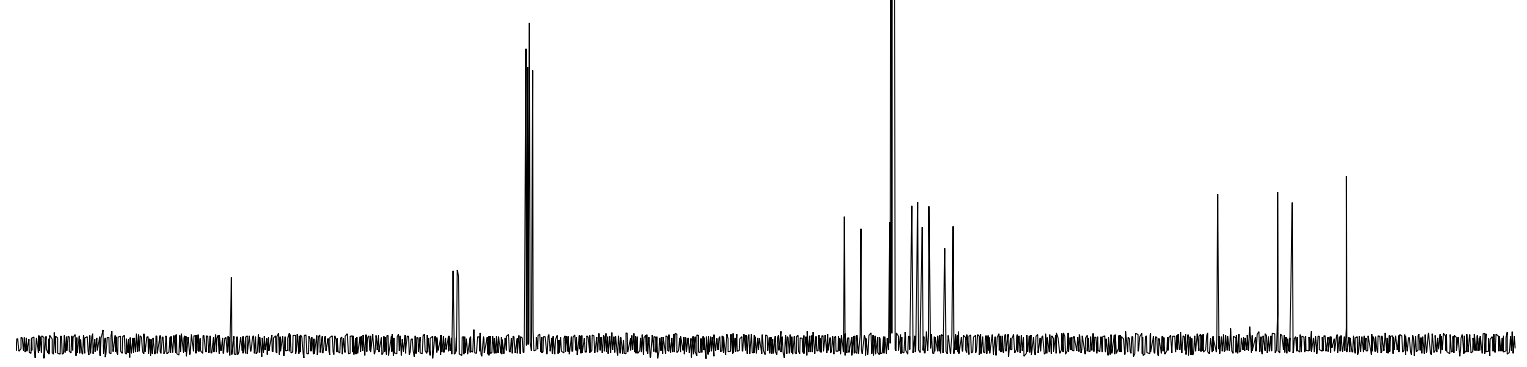

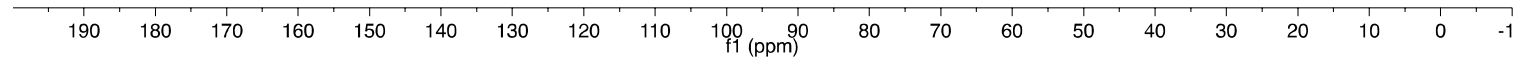

${ }^{13} \mathrm{CNMR}\left(\mathrm{CDCl}_{3}, 151 \mathrm{MHz}\right)$ 


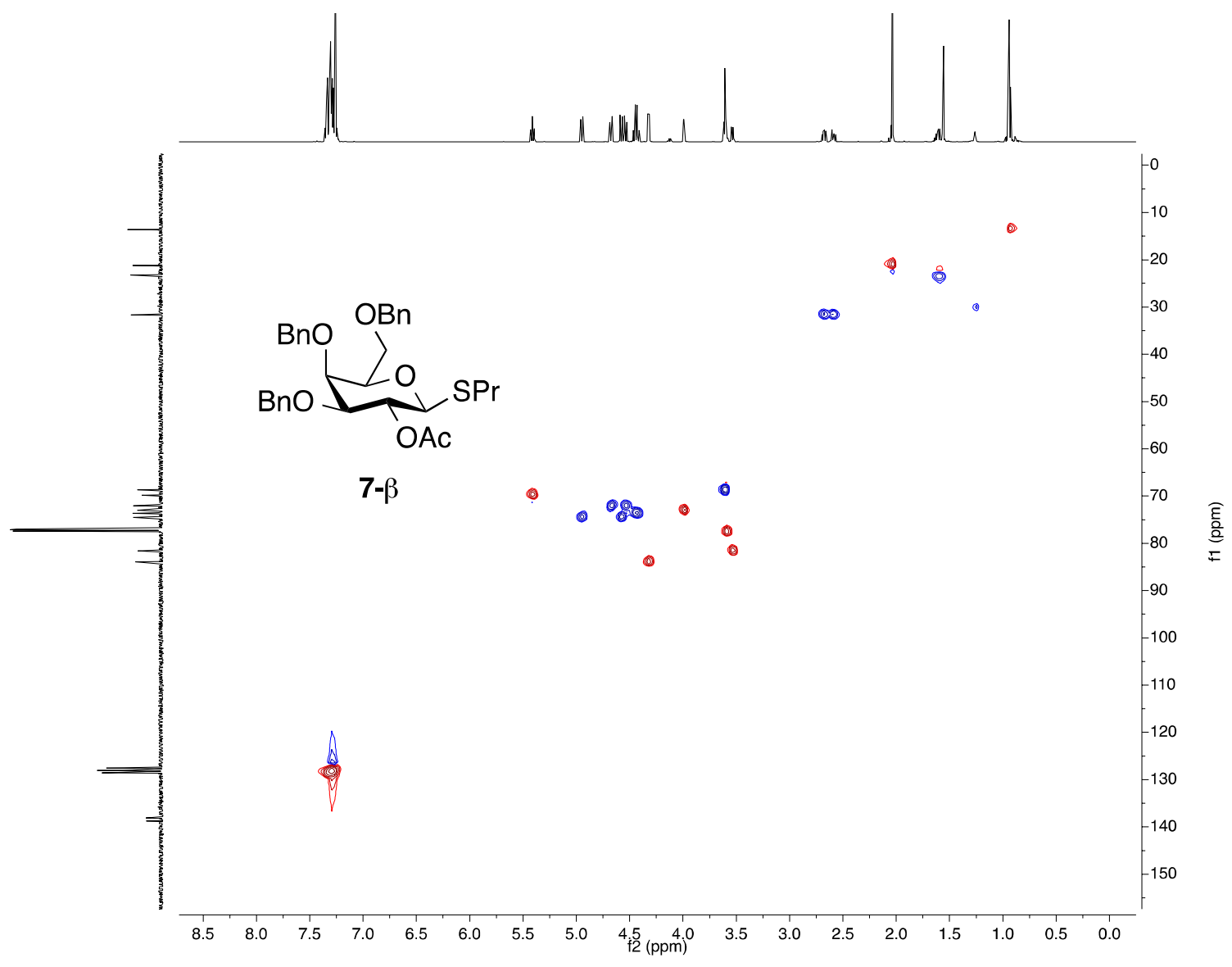

HSQC-phase edit $\left(\mathrm{CDCl}_{3}\right)$ 


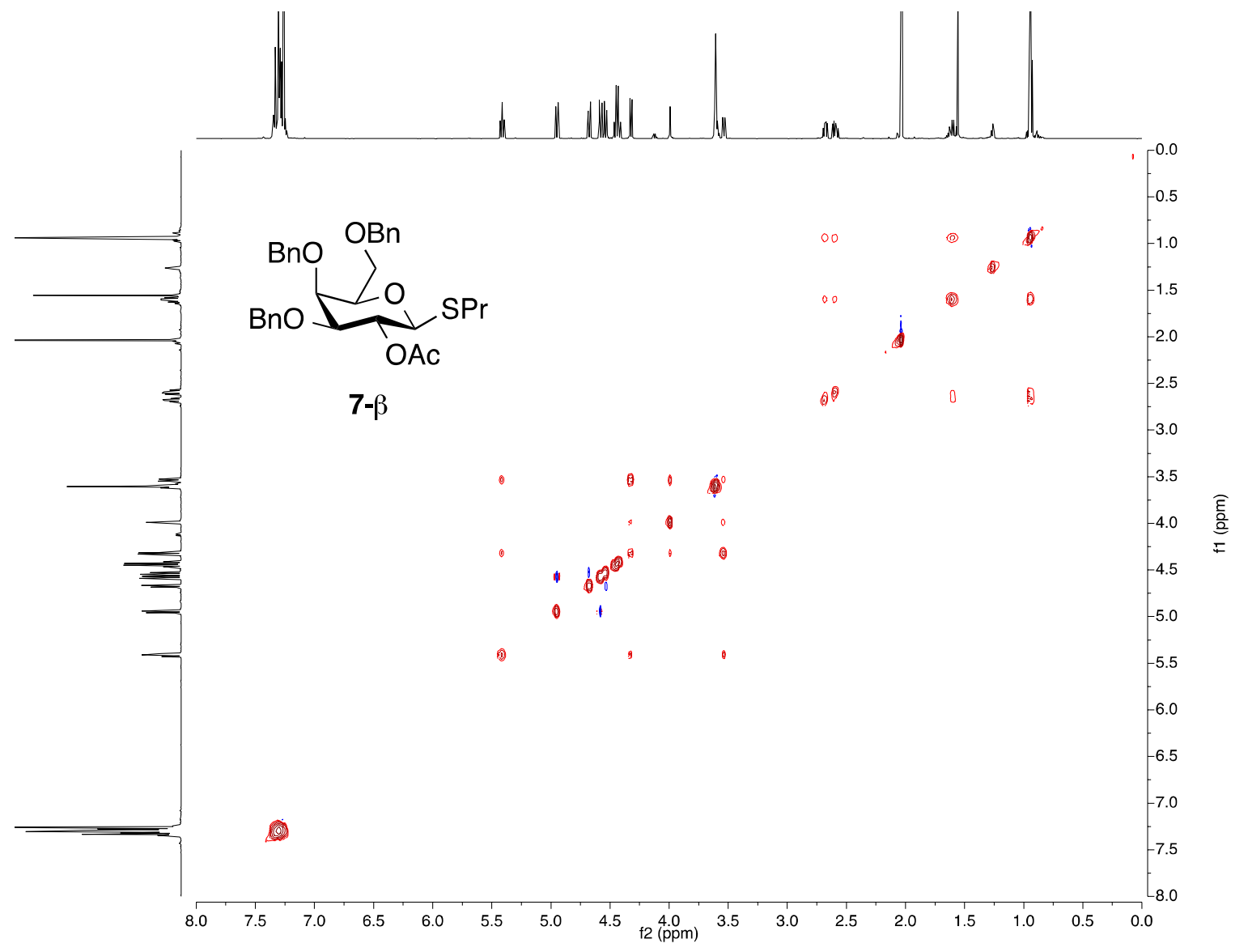

TOCSY $\left(\mathrm{CDCl}_{3}, 600 \mathrm{MHz}\right)$ 


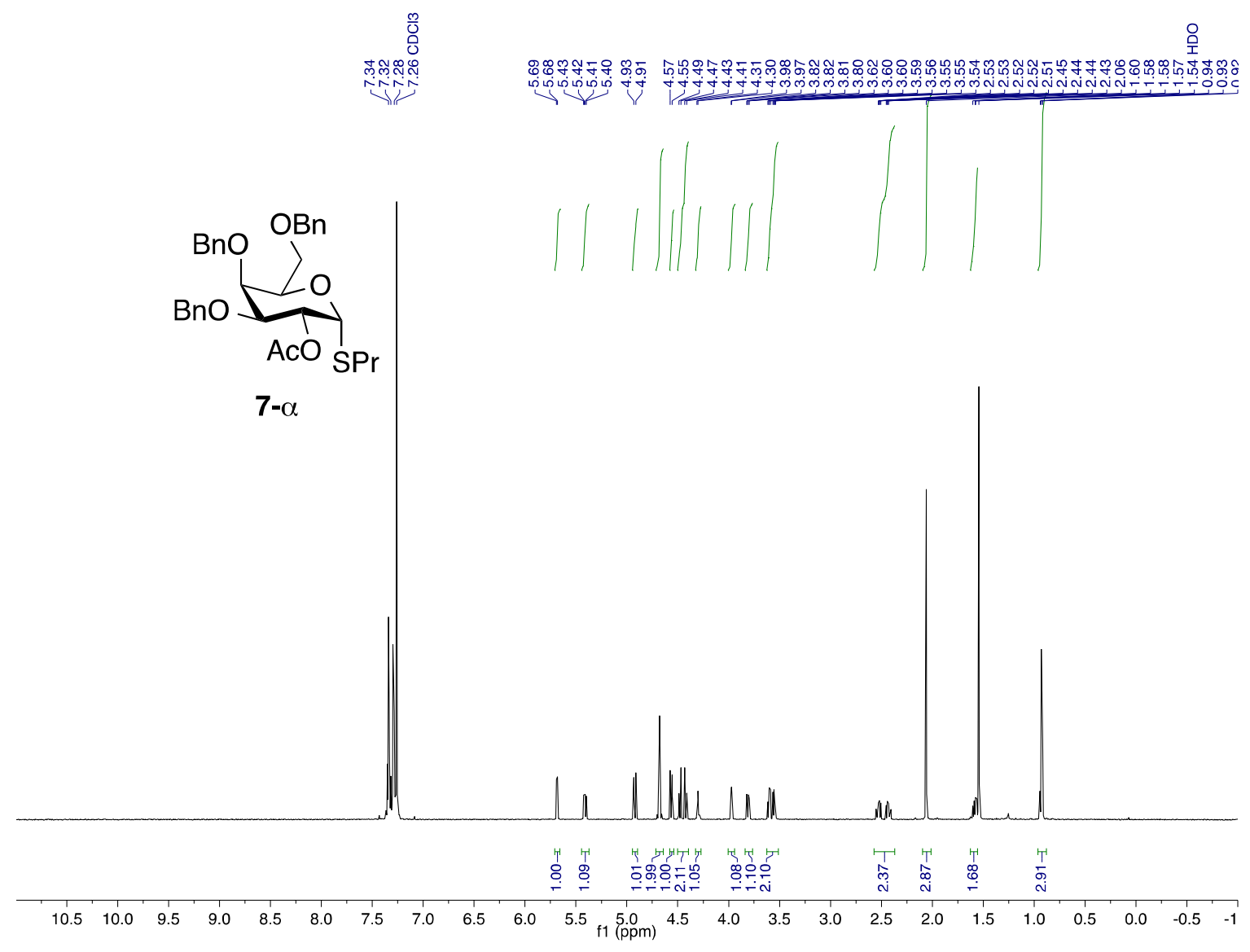

${ }^{1} \mathrm{HNMR}\left(\mathrm{CDCl}_{3}, 600 \mathrm{MHz}\right)$ 


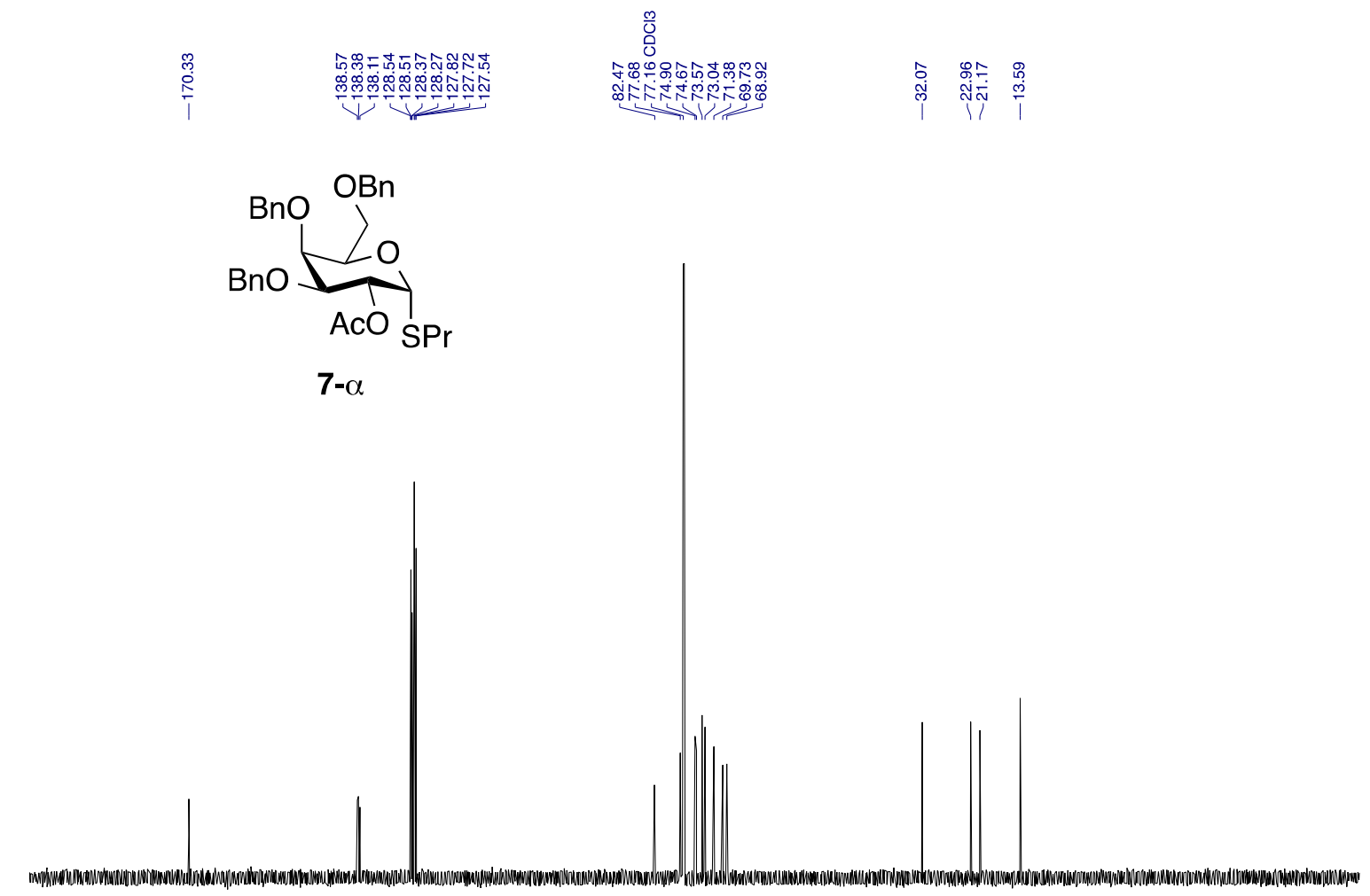

\begin{tabular}{lllllllllllllllllllllllllllll}
\hline 0 & 190 & 180 & 170 & 160 & 150 & 140 & 130 & 120 & 110 & 100 & 90 & 80 & 70 & 60 & 50 & 40 & 30 & 20 & 10 & 0 & -10 & -20 & -30 & -40 & -5
\end{tabular}

${ }^{13} \mathrm{CNMR}\left(\mathrm{CDCl}_{3}, 151 \mathrm{MHz}\right)$ 


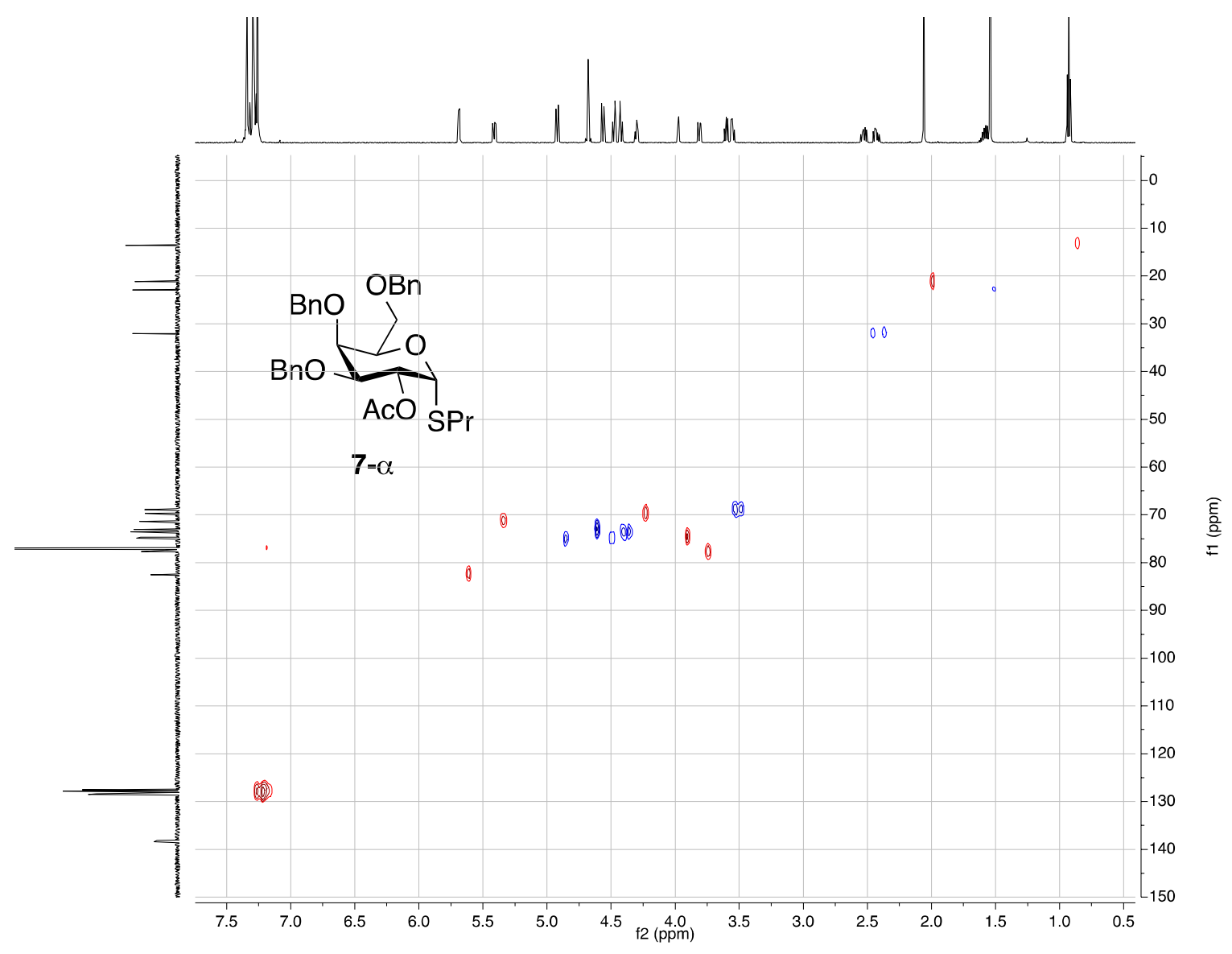

HSQC-phase edit $\left(\mathrm{CDCl}_{3}\right)$ 


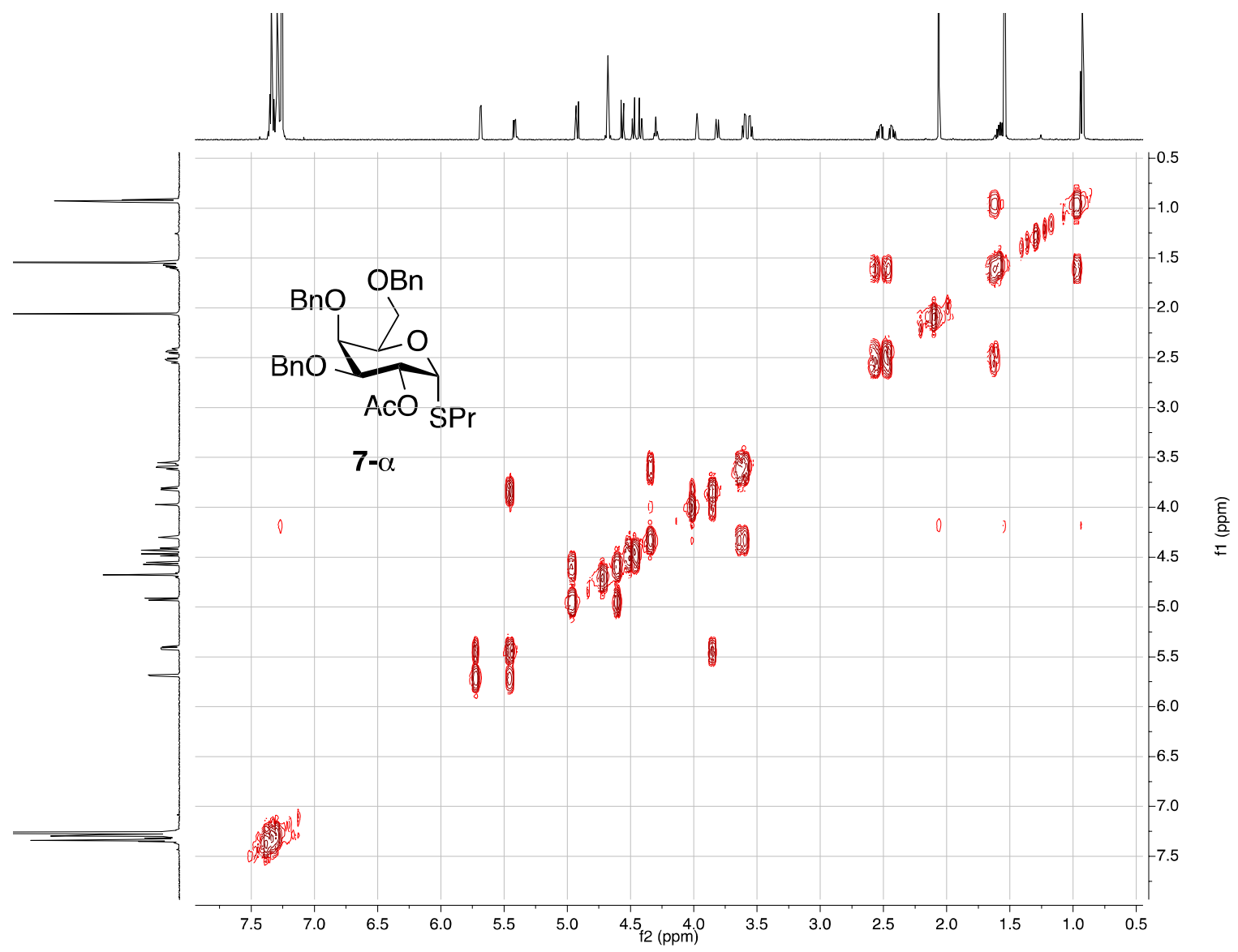

${ }^{1} \mathrm{H}-{ }^{1} \mathrm{H} \operatorname{COSY}\left(\mathrm{CDCl}_{3}, 600 \mathrm{MHz}\right)$ 

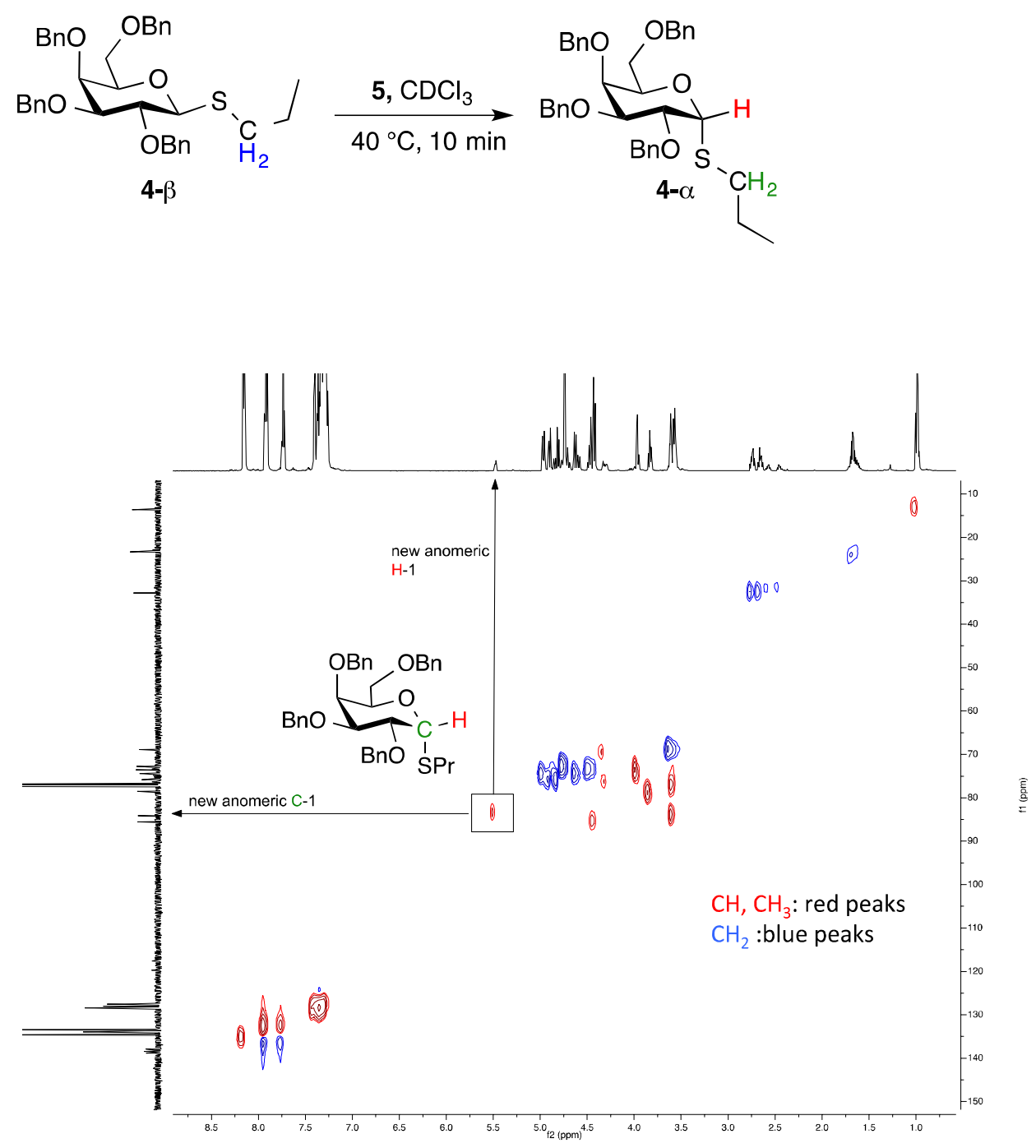

HSQC phase edit $\left(\mathrm{CDCl}_{3}\right)$ 


\section{Reaction kinetics spectra}

1)<smiles>CCCS[Sb](Br)(Br)c1ccccc1</smiles>

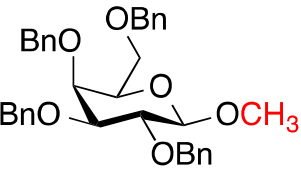

Donor $(4 \alpha)$

$\mathrm{Ph}_{3} \mathrm{Bi}(\mathrm{OTf})_{2}$

$+$

$\underset{\text { Promoter (1) }}{\longrightarrow}$

$(6 \alpha)$

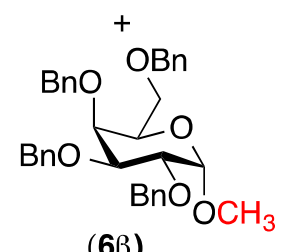

Acceptor (5)

(6ß)

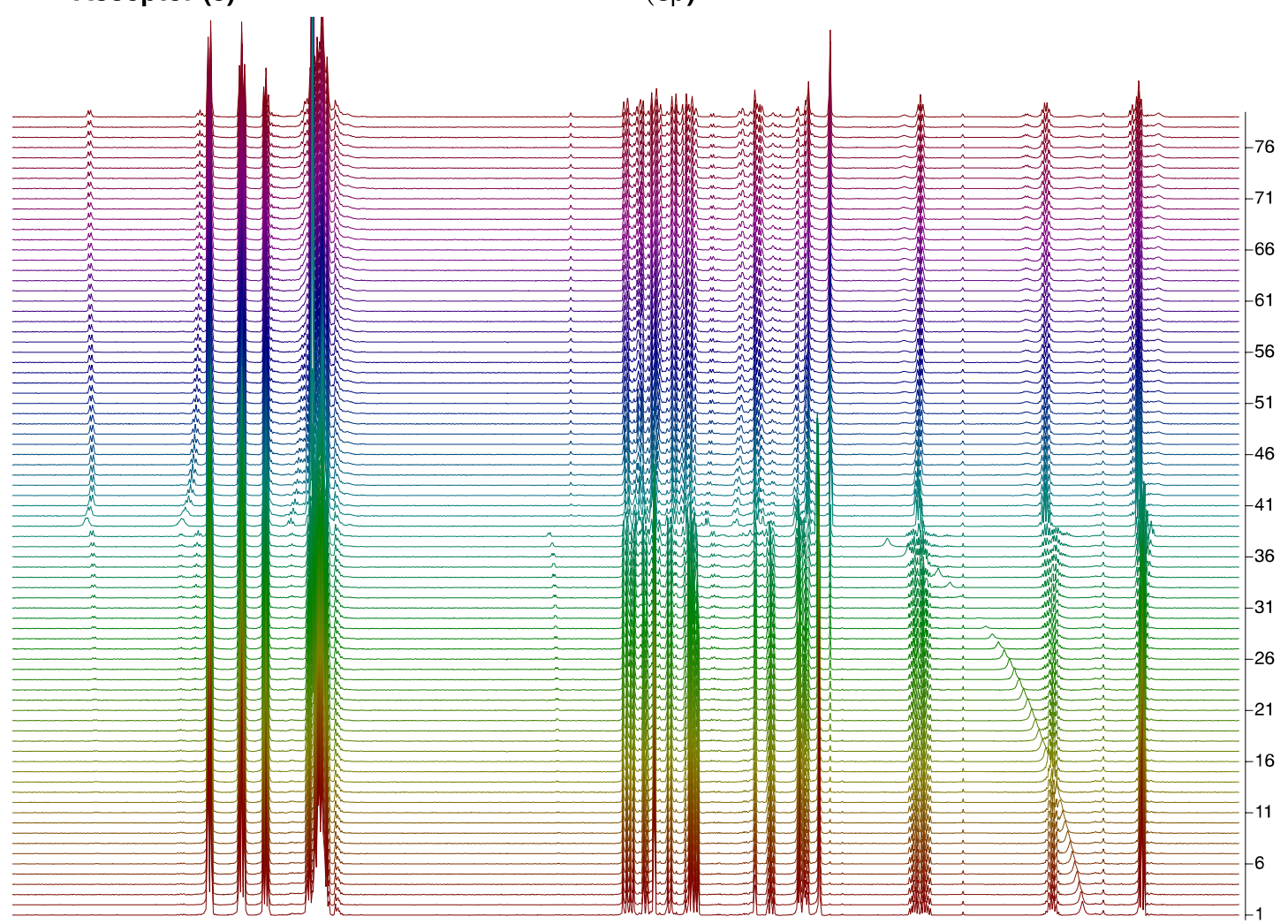

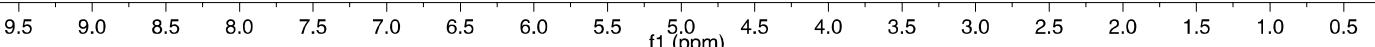


2)
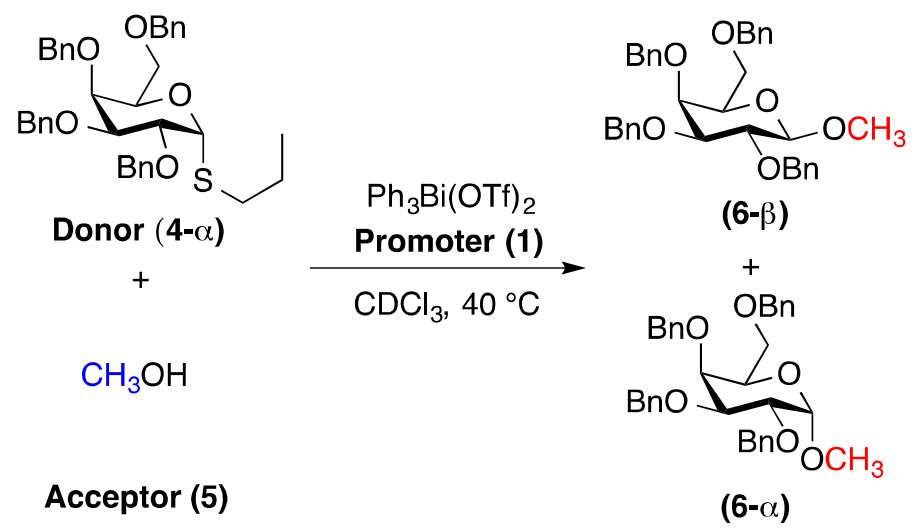

Acceptor (5)

(6- $\alpha)$

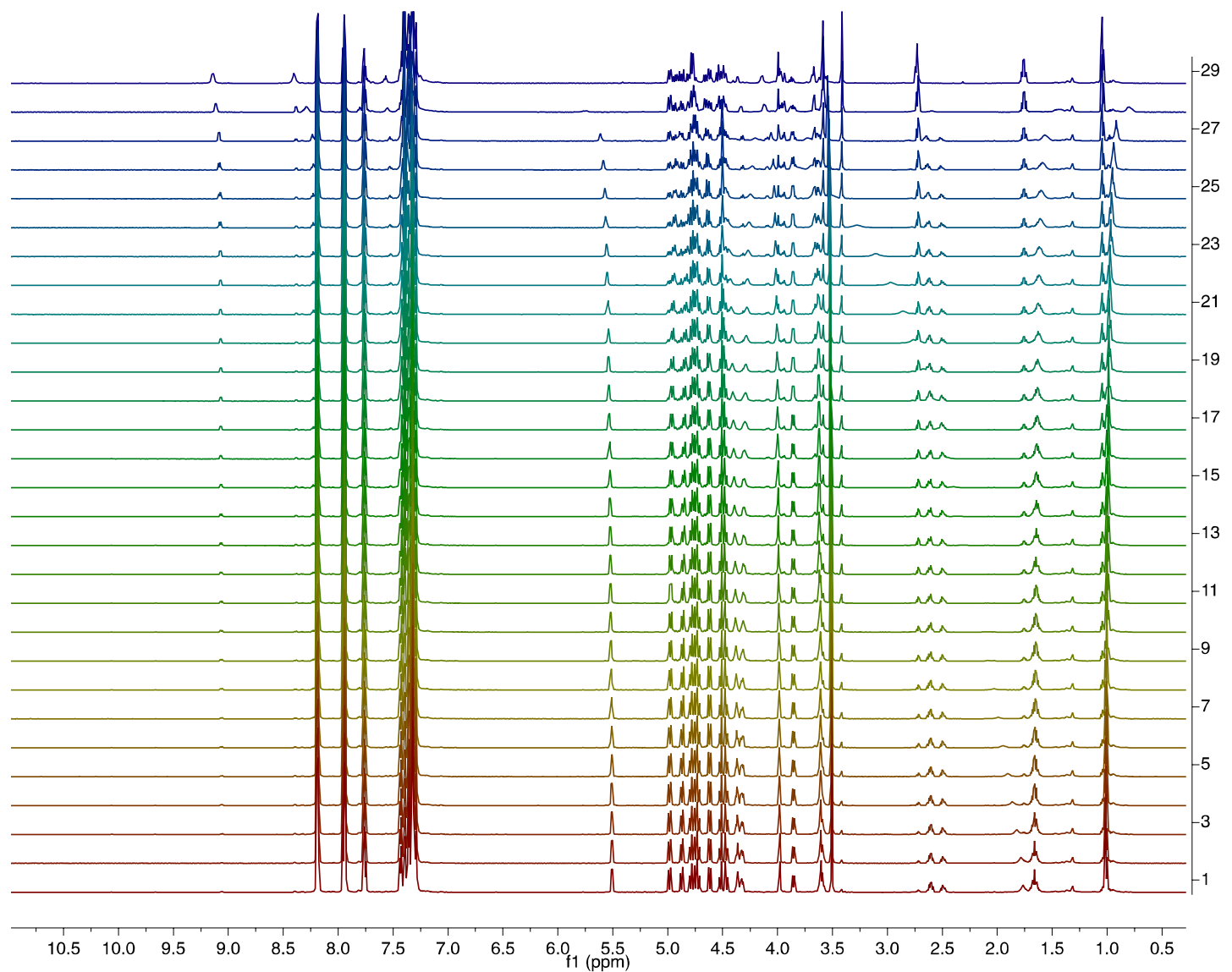




\section{Computational Methodology}

Calculations were carried out using DFT as implemented in the Jaguar 8.1 suite of ab initio quantum chemistry programs. ${ }^{1}$ MP2 and HF calculations were carried out with the ORCA electronic structure package, version 3.0.0. ${ }^{2}$ Geometry optimizations were performed with the hybrid functional B3LYP ${ }^{3-6}$ using Grimme's D3 ${ }^{7-8}$ correction for dispersion (referred to as B3LYPD3) unless otherwise noted. The 6-31G** basis set was used for all light atoms. ${ }^{9-10} \mathrm{Bi}$ was represented using the Los Alamos LAV3P basis sat that includes relativistic effective core potentials. ${ }^{11}$ This basis set is essentially a modified version of LAV2P, in which the exponents were decontracted to match the effective core potential with triple- $\zeta$ quality. The energies of the optimized structures were reevaluated by additional single point calculations on each optimized geometry using Dunning's correlation consistent triple- $\zeta$ basis set cc-pVTZ(-f $)^{12-13}$ that includes a double set of polarization functions on all light atoms, in addition to the aforementioned LAV3P on $\mathrm{Bi}$. For our benchmarking studies using MP2 and HF we employed slightly different, but entirely comparable, basis sets: LANL08 ${ }^{14}$ (with effective core potentials ${ }^{11}$ ) on $\mathrm{Bi}$, and cc-pVTZ on all other atoms. ${ }^{12}$ Additional single points were also calculated with the inclusion of diffuse functions ${ }^{13,15}$ to accurately model anions and the softer, polarizable sulfur atoms. We found that addition of diffuse functions only made an appreciable difference for calculating reactions that involved anions (ex: triflate dissociation). As they are much more expensive we then did not employ them for our relaxed scans, or our benchmarking studies. Analytical vibrational frequencies were calculated within the harmonic approximation at the double- $\zeta$ level to ensure that all structures reported were well-defined minima with no imaginary frequencies.

Vibrational/rotational/translational entropies of the solute(s) were included using standard thermodynamic approximations. Solvation energies were evaluated by a selfconsistent reaction field (SCRF) approach based on accurate numerical solutions of the linearized Poisson-Boltzmann equation. ${ }^{16-19}$ Solvation calculations were performed at the optimized gas phase geometries employing the dielectric constant of $\varepsilon=4.806$ (chloroform). As is the case for all continuum models, the solvation energies are subject to empirical parameterization of the atomic radii that are used to generate the solute surface. We employ the standard set of optimized radii for $\mathrm{H}(1.150 \AA)$, C (1.900 $)$, O (1.600 $\AA$ ), Bi $(2.185 \AA$ ), S $(1.900 \AA)$, and $F(1.682 \AA)$. Energy components were computed following the protocol of our previous work. The change in solution phase free energy, $\Delta G($ sol), can be calculated as follows:

$$
\begin{gathered}
\Delta \mathrm{G}(\text { sol })=\Delta \mathrm{G}(\text { gas })+\Delta \Delta \mathrm{G}_{\text {solv }} \\
\Delta \mathrm{G}(\text { gas })=\Delta \mathrm{H}(\text { gas })-\mathrm{T} \Delta \mathrm{S} \text { (gas) }
\end{gathered}
$$




$$
\Delta H(\text { gas })=\Delta E(S C F)+\Delta Z P E
$$

$\Delta G($ gas $)=$ change in gas phase free energy; $\Delta \Delta G_{\text {solv }}=$ change in free energy of solvation; $\Delta H$ (gas) = change in gas phase enthalpy; $T$ = temperature (313.15 $\mathrm{K}) ; \Delta \mathrm{S}($ gas $)=$ change in gas phase entropy; $\triangle E(S C F)=$ self consistent field energy, i.e. "raw" electronic energy as calculated the triple- $\zeta$ level (with diffuse function corrections); $\triangle \mathrm{ZPE}=$ change in vibrational zero point energy.

To properly evaluate the energy of different thioglycoside species we had to attempt to locate the lowest energy conformer for each species, or at least one of the lowest energy conformers. Our geometry optimizations themselves only confirm that we have obtained a local minima, which will not necessarily be the global minima. We accomplished this by using the Global-MMX program (GMMX) as employed by PCModel. ${ }^{20}$ This conformational analysis employed the MMX force field ${ }^{21}$ and generated a large number of reasonably low energy conformers. While this analysis is useful for generating a small subset of reasonable structures, these structures and energies will not be accurate enough for our analysis. These structures were therefore re-optimized with a DFT functional. We initially employed M06, ${ }^{22}$ and maintained this throughout our study for the conformational analysis. As shown in our benchmarking studies (see the main text of the manuscript) M06 also performs reasonably well, although not as well as B3LYP-D3. Single point electronic energy calculations (triple-乙, no diffuse functions) were performed on the lowest energy structures to confirm that the lowest energy structure at the geometry optimization level was in fact correct. This structure was then re-optimized with B3LYP-D3 and taken to be the global minima. One exception to this protocol was that the MMX calculations consistently re-optimized trigonal bipyramidal five-coordinate structures (such as 10) to square pyramidal geometries that were inconsistent with the DFT calculations. Therefore to perform a conformational analysis on these structures we used the GMMX results of four coordinate structures (see S5 and S6 in Figure S2) and manually added triflate ions to these prior to DFT re-optimization.

There are many assumptions in this study, including deciding the lowest energy structure by electronic energy alone, and assuming that the MMX force fields will be accurate enough for a conformational analysis. For the former it was not computationally affordable to calculate thermochemical effects (entropy and ZPE) for all possible conformers due to the sheer volume of structures generated. As to the accuracy of the force fields, MMX is no doubt reasonably accurate for the organic fragments, but certainly can't be as well parameterized for $\mathrm{Bi}$. In fact we noted early on that initially modelling $\mathrm{Bi}$ as $\mathrm{Pb}$ with $\mathrm{GMMX}$ produced significantly lower energy structures after DFT optimization than when Bi force fields were used. Overall then this GMMX methodology is not intended to be a completely accurate method of 
conformational analysis, but rather serves as a very reasonable method of generating structures that are no doubt close in energy to the global minima, if not the global minima themselves.

There are a few useful examples demonstrating these issues. As noted in the text, the energies of 4- $\boldsymbol{\alpha}$ and $\mathbf{4 - \boldsymbol { \beta }}$ are essentially identical electronically, but $\mathbf{4 - \alpha}$ is entropically higher in energy. There was some concern that this effect was arising only because we chose the lowest energy conformer based on electronic energy, and not the solvated free energy. To test this, we calculated entropic and solvation corrections for the 8 lowest energy conformers for 4- $\boldsymbol{\alpha}$ and $4-\boldsymbol{\beta}$ to see if inclusion of these corrections dramatically changed the anomerization energy. By including entropy, ZPE, and solvation terms to 4- $\boldsymbol{\alpha}$ one of the conformers that was slightly higher in energy electronically was now lower in energy at the $G($ sol) (no diffuse functions) level by $1.2 \mathrm{kcal} / \mathrm{mol}$. However applying the same analysis to $4-\boldsymbol{\beta}$ yielded a similar result, by finding a new conformer that was now lower in energy by $1.5 \mathrm{kcal} / \mathrm{mol}$ (again at the $\mathrm{G}(\mathrm{sol})$ level). This essentially preserves the difference in energy between $\mathbf{4 - \alpha}$ and $\mathbf{4 - \boldsymbol { \beta }}$ and for the same reasons; they are still electronically isoenergetic and the main component of their energy differences are entropic: $4-\alpha$ is $\sim 2 \mathrm{kcal} / \mathrm{mol}$ higher in energy than $\mathbf{4}-\boldsymbol{\beta}$ due to entropy for each pair of anomers. The structures and energies we report for $4-\alpha$ and $4-\beta$ are the ones originally located from our conformational analysis, i.e. based on electronic energy. This was done to be more systematic by always choosing the lowest energy structure based on electronic energy, and because the results were essentially the same regardless (1-2 kcal/mol is easily within the DFT error limit).

We also note that during our relaxed scans for thiolate abstraction (see text) we found a lower energy isomer for $\mathbf{1 0}-\boldsymbol{\beta}$ (by $2.05 \mathrm{kcal} / \mathrm{mol}$ electronically, and $1.25 \mathrm{kcal} / \mathrm{mol}$ in terms of solvated free energy). We used this lower energy structure, but it is quite clear that the original structure and energy would have led to all the same relevant chemical conclusions. Overall these examples confirm what was stated above: that there are some flaws in our conformational analysis, but that there actual effects on our chemical conclusions are most likely minimal. 


\section{Calculated Energies}

Figure S1. Reaction energies $(\Delta G(\mathrm{sol}))$ for additional thiolate abstraction reactions mentioned in the text. All energies are in $\mathrm{kcal} / \mathrm{mol}$.
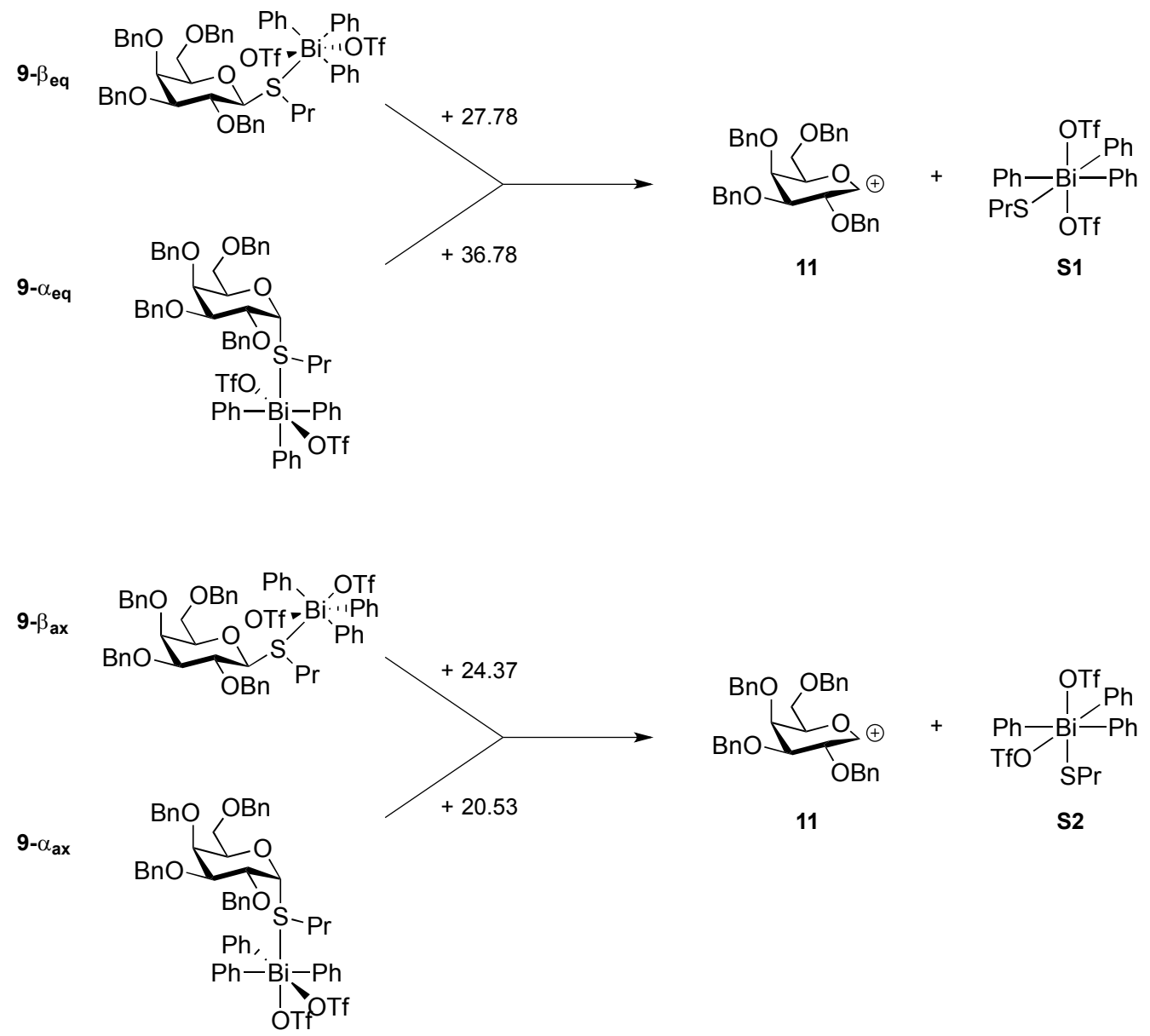
Figure S2. Reaction energies $(\Delta \mathrm{G}(\mathrm{sol}))$ for additional triflate dissociation reactions mentioned in the text. All energies are in $\mathrm{kcal} / \mathrm{mol}$.

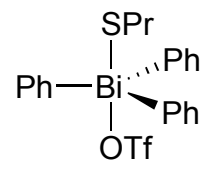

S3

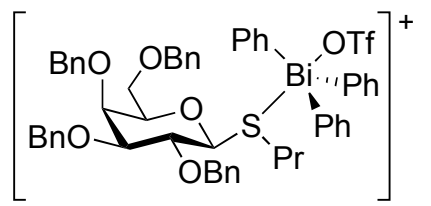

10- $\beta$

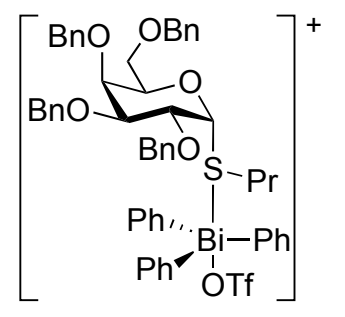

$10-\alpha$

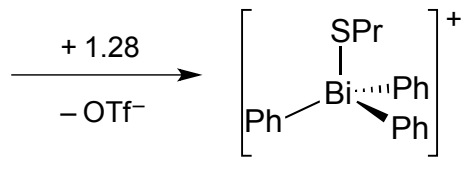

S4
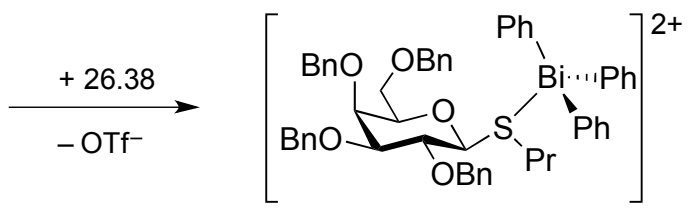

S5

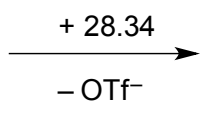

S6 
Table S1. Triple- $\zeta$ single point energies at zero Kelvin (E(SCF)a), Triple- $\zeta$ single point energies at zero Kelvin with diffuse functions applied $(E(S C F))$, zero point energies (ZPE), entropy contribution (TS) at $313.15 \mathrm{~K}$, solvation energies ( $\Delta \mathrm{G}($ solv)) and the total free energy with diffuse functions ( $\mathrm{G}(\mathrm{sol})$ of all relevant structures in $\mathrm{kcal} / \mathrm{mol}$.

\begin{tabular}{|c|c|c|c|c|c|c|}
\hline Compound & $E(S C F) a$ & $\mathrm{E}(\mathrm{SCF})$ & ZPE & -TS & $\Delta G($ solv $)$ & G(sol) \\
\hline 1 & -1646593.97 & -1646610.56 & 206.86 & -73.24 & -11.49 & -1646488.43 \\
\hline $1 a$ & -1042951.73 & -1042961.56 & 188.20 & -59.64 & -34.59 & -1042867.58 \\
\hline $1 \mathrm{~b}$ & -439219.39 & -439222.28 & 169.34 & -46.12 & -126.02 & -439225.08 \\
\hline$M 1_{a x}$ & -1946596.26 & -1946614.18 & 255.58 & -81.39 & -15.03 & -1946455.02 \\
\hline S1 & -1970970.43 & -1970990.63 & 266.41 & -83.80 & -36.78 & -1970844.80 \\
\hline S2 & -1970980.34 & -1970999.76 & 266.48 & -79.37 & -39.75 & -1970852.40 \\
\hline S3 & -1367424.90 & -1367435.95 & 249.00 & -69.69 & -11.03 & -1367267.66 \\
\hline S4 & -763802.90 & -763806.85 & 230.67 & -58.46 & -32.32 & -763666.96 \\
\hline OTf & -603528.90 & -603540.50 & 17.09 & -27.21 & -48.8 & -603599.42 \\
\hline $\mathrm{Me}_{2} \mathrm{~S}$ & -300001.14 & -300002.04 & 47.65 & -21.91 & -2.49 & -299978.79 \\
\hline $9-\beta_{a x}$ & -3033536.09 & -3033560.51 & 658.63 & -135.15 & -16.6 & -3033053.64 \\
\hline $9-\alpha_{a x}$ & -3033531.25 & -3033556.52 & 658.93 & -133.97 & -18.23 & -3033049.79 \\
\hline $9-\beta_{\text {eq }}$ & -3033534.06 & -3033558.44 & 658.34 & -133.07 & -16.27 & -3033049.44 \\
\hline $9-\alpha_{\mathrm{eq}}$ & -3033543.45 & -3033568.17 & 659.14 & -133.02 & -16.39 & -3033058.44 \\
\hline $4-\beta$ & -1386927.75 & -1386935.41 & 451.26 & -78.97 & -10.32 & -1386573.44 \\
\hline $4-\alpha$ & -1386927.66 & -1386935.06 & 451.90 & -76.88 & -10.53 & -1386570.57 \\
\hline $10-\beta$ & -2429925.37 & -2429942.09 & 641.63 & -118.32 & -34.04 & -2429452.83 \\
\hline $10-\alpha$ & -2429918.29 & -2429935.75 & 641.03 & -121.38 & -34.49 & -2429450.59 \\
\hline $10_{\mathrm{En}}$ & -2429895.74 & -2429912.39 & 639.48 & -121.40 & -40.93 & -2429435.24 \\
\hline $10-\beta_{c h}$ & -2429922.35 & -2429936.56 & 641.39 & -117.70 & -34.73 & -2429447.60 \\
\hline S5 & -1826238.95 & -1826247.50 & 622.93 & -106.46 & -95.99 & -1825827.03 \\
\hline S6 & -1826235.90 & -1826246.28 & 622.96 & -105.20 & -94.31 & -1825822.83 \\
\hline 11 & -1062458.07 & -1062464.64 & 390.63 & -67.09 & -35.76 & -1062176.86 \\
\hline
\end{tabular}


Table S2. Triple- $\zeta$ electronic energies calculated with different computational methods used for methodology benchmarking. Structures were optimized with B3LYP unless otherwise indicated. All energies are in kcal/mol. ${ }^{a}$ Structure was optimized with B3LYP-D3.

\begin{tabular}{|c|c|c|c|c|c|}
\hline Compound & HF & B3LYP & B3LYP-D3 & M06 & MP2 \\
\hline $1 \mathrm{a}(\mathrm{Me})$ & -679192.53 & -681871.80 & -681882.53 & -681626.92 & -680724.75 \\
\hline $1 \mathrm{a}(\mathrm{Ph})$ & -1037938.66 & -1042924.06 & -1042951.64 & -1042394.15 & -1040914.22 \\
\hline $1 \mathrm{a}(\mathrm{Ph})^{\mathrm{a}}$ & -1037938.03 & -1042923.60 & -1042951.73 & -1042394.27 & -1040914.86 \\
\hline 4- $\alpha(\mathrm{MeO})$ & -802906.33 & -806732.43 & -806763.91 & -806335.93 & -805163.32 \\
\hline $4-\beta(\mathrm{MeO})$ & -802905.96 & -806731.04 & -806762.43 & -806334.77 & -805162.05 \\
\hline 4- $\alpha(B n O)^{a}$ & -1379244.75 & -1386847.87 & -1386927.66 & -1386004.18 & -1383880.65 \\
\hline 4- $\beta(B n O)^{a}$ & -1379240.61 & -1386843.65 & -1386927.75 & -1386002.95 & -1383880.16 \\
\hline 10- $\alpha(\mathrm{MeO}, \mathrm{Me})$ & -1482129.041 & -1488636.82 & -1488694.28 & -1488008.55 & -1485933.45 \\
\hline $10-\beta(\mathrm{MeO}, \mathrm{Me})$ & -1482127.44 & -1488634.16 & -1488692.12 & -1488007.22 & -1485933.06 \\
\hline $10-\alpha(\mathrm{MeO}, \mathrm{Ph})$ & -1840857.61 & -1849674.01 & -1849755.88 & -1848764.97 & -1846119.07 \\
\hline $10-\beta(\mathrm{MeO}, \mathrm{Ph})$ & -1840859.032 & -1849673.415 & -1849753.389 & -1848763.33 & -1846117.595 \\
\hline $10-\alpha(B n O, P h)^{a}$ & -2417191.99 & -2429786.41 & -2429918.29 & -2428431.47 & $\mathrm{~N} / \mathrm{A}$ \\
\hline $10-\beta(\mathrm{BnO}, \mathrm{Ph})^{\mathrm{a}}$ & -2417185.31 & -2429781.22 & -2429925.37 & -2428437.95 & $\mathrm{~N} / \mathrm{A}$ \\
\hline
\end{tabular}

\section{DFT Methodology Benchmarking}

Given the importance of the dispersion interactions present in this system, it was important to verify that our computational methodology was accurately capturing these effects. We performed a series of benchmark calculations to evaluate B3LYP, B3LYP-D3, and $\mathrm{M06}^{22}$ (another DFT functional that is parameterized to accurately capture dispersion ${ }^{22}$ ) by comparing them with ab initio MP2 calculations. This data is shown in Table S3. We performed most of our benchmark calculations on smaller model systems for computational cost purposes, by substituting the benzyl groups on the thioglycoside with methoxy groups and the $\mathrm{Ph}$ groups on the Bi promoter with methyl groups (see Figure $\mathrm{S} 3$ ). These small models were optimized with the standard B3LYP functional. As these models were only being used to verify the level of theory, and not to gain chemical insights into the nature of the lowest energy structure, conformational analysis was not performed for these small models. In general, we found that MP2 calculations agree quite well with B3LYP-D3 and to a lesser extent M06, but in many instances neglecting these dispersion interactions by using a standard functional like B3LYP offered significantly worse agreement. 
Figure S3. Generic models and nomenclature used for methodology benchmarking calculations. Binding reaction for $\mathbf{4}-\boldsymbol{\beta}$ used as an example.
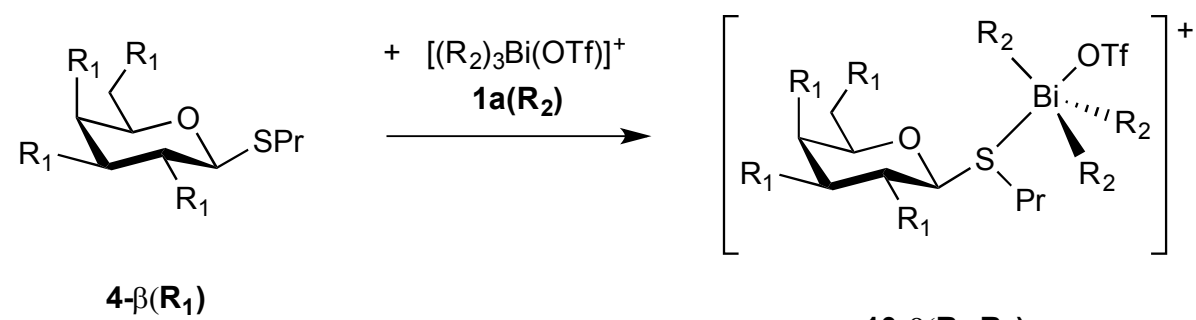

$10-\beta\left(\mathbf{R}_{1}, \mathbf{R}_{2}\right)$

Table S3. Methodology benchmarking calculations. All reported values are electronic energies in $\mathrm{kcal} / \mathrm{mol}$ at the triple- $\zeta$ level of theory (see SI for more details). All structures are optimized with B3LYP with the exception of when $\mathrm{R}_{1}=\mathrm{BnO}$ which were optimized with B3LYP-D3, as these are our actual structures referred to throughout the paper. ${ }^{a}$ Refers to the substituents on the thioglycoside. ${ }^{b}$ Refers to the substituents on the Bi promoter. ${ }^{c}$ Energy of going from the $\beta$ anomer to the $\alpha$-anomer of the free thioglycoside (analogous to 4). ${ }^{d}$ Energy of going from the $\beta$-anomer to the $\alpha$-anomer of the 5 -coordinate Bi-adduct (analogous to 10). ${ }^{\mathrm{e}}$ Binding energy for the 5 -coordinate $\mathrm{Bi}$-adduct (analogous to 10 ) for the respective anomer with 1 a. See Figure S3 for more details.

\begin{tabular}{llllllll}
\hline Reaction & $\mathrm{R}_{1}{ }^{\mathrm{a}}$ & $\mathrm{R}_{2}{ }^{\mathrm{b}}$ & $\mathrm{HF}$ & $\mathrm{B}$ LLYP & B3LYP-D3 & $\mathrm{M} 06$ & $\mathrm{MP2}$ \\
\hline Thioglycoside $^{\mathrm{c}}$ & $\mathrm{MeO}$ & $\mathrm{N} / \mathrm{A}$ & -0.36 & -1.38 & -1.48 & -1.15 & -1.27 \\
$(\beta \rightarrow \alpha)$ & $\mathrm{BnO}$ & $\mathrm{N} / \mathrm{A}$ & -4.14 & -4.22 & 0.09 & -1.22 & -0.48 \\
\hline Bi- Adduct $^{\mathrm{d}}$ & $\mathrm{MeO}$ & $\mathrm{Me}$ & -1.60 & -2.65 & -2.17 & -1.34 & -0.39 \\
$(\beta \rightarrow \alpha)$ & $\mathrm{MeO}$ & $\mathrm{Ph}$ & 1.42 & -0.60 & -2.49 & -1.64 & -1.48 \\
& $\mathrm{BnO}$ & $\mathrm{Ph}$ & -6.68 & -5.18 & 7.08 & 6.48 & $\mathrm{~N} / \mathrm{A}$ \\
\hline Binding $(\beta)^{\mathrm{e}}$ & $\mathrm{MeO}$ & $\mathrm{Me}$ & -28.95 & -31.32 & -47.16 & -45.52 & -46.26 \\
& $\mathrm{MeO}$ & $\mathrm{Ph}$ & -14.40 & -18.31 & -39.32 & -34.41 & -41.32 \\
& $\mathrm{BnO}$ & $\mathrm{Ph}$ & -6.67 & -13.97 & -45.89 & -40.72 & $\mathrm{~N} / \mathrm{A}$ \\
\hline Binding $(\alpha)^{\mathrm{e}}$ & $\mathrm{MeO}$ & $\mathrm{Me}$ & -30.18 & -32.58 & -47.85 & -45.71 & -45.38 \\
& $\mathrm{MeO}$ & $\mathrm{Ph}$ & -12.62 & -17.53 & -40.33 & -34.89 & -41.53 \\
& $\mathrm{BnO}$ & $\mathrm{Ph}$ & -9.22 & -14.94 & -38.90 & -33.02 & $\mathrm{~N} / \mathrm{A}$ \\
\hline
\end{tabular}

For comparing the energies of $\beta$ - and $\alpha$-anomers, all methods agreed reasonably well for the small model thioglycoside. However for the larger benzylated thioglycosides, B3LYP starkly disagreed with the dispersion-corrected methods, and actually only performed as well as HF. For the free thioglycosides, it substantially over stabilized the $\alpha$-anomer by $\sim 4$ $\mathrm{kcal} / \mathrm{mol}$. Given that one of the key results of our calculations was that the $\beta$ - and $\alpha$ - 
thioglycosides were similar in energy, this result would have been lost without including dispersion corrections. Even more dramatic however was the $\beta$ and $\alpha$ energy difference for the 5 -coordinate $\mathrm{Bi}$-adduct. Here B3LYP and HF predict the $\alpha$-anomer to be more stable than the $\beta$ anomer by $\sim 6 \mathrm{kcal} / \mathrm{mol}$, while B3LYP-D3 and M06 predict the opposite leading to a difference of $\sim 12 \mathrm{kcal} / \mathrm{mol}$ for the two methods. No matter which model was used however, dispersion was absolutely critical for predicting accurate binding energies. We modeled the binding energy of 1a to 4 to form the 5-coordinate adduct 10. This reaction serves as a reasonable model for how well each method treats the interaction between the $\mathrm{Bi}$ fragment and the thioglycoside fragment. B3LYP routinely underestimated the binding energy ranging from $\sim 15-35 \mathrm{kcal} / \mathrm{mol}$ less favorable compared to the dispersion-corrected methods. Note that for these reactions, the previously mentioned agreement with HF diminished as the models got larger. Overall, these benchmarking calculations indicated that our treatment of dispersion with B3LYP-D3 was reasonably accurate. They also highlight the importance of careful method evaluation when dealing with large molecules like carbohydrates, where dispersion interactions can be critical. 


\section{Cartesian Coordinates of}

\section{Optimized Structures}

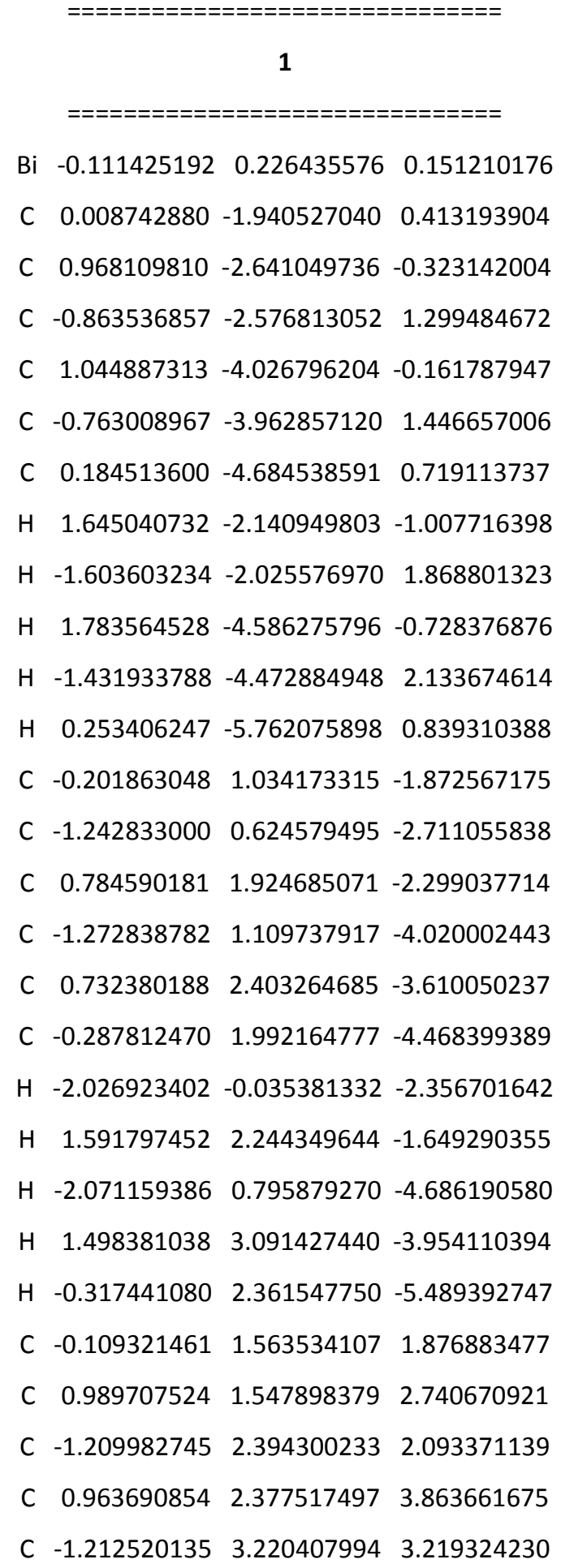

C $-0.133562708 \quad 3.206760155 \quad 4.104069664$

H $\quad \begin{array}{llll}\text { H } & 1.855976544 & 0.927178377 & 2.541587122\end{array}$

H $-2.0598771312 .411549831 \quad 1.420418637$

H $1.806831642 \quad 2.3725593424 .548240892$

H $-2.066009602 \quad 3.866500807 \quad 3.400645276$

H $\quad-0.146207303 \quad 3.845580408 \quad 4.982392497$

$\begin{array}{llll}\text { O } & 2.092359581 & 0.375655278 & 0.245654245\end{array}$

$\begin{array}{llll}\text { O } & -2.320808370 & 0.208342626 & 0.027775217\end{array}$

S $3.2938523610 .285347504-0.725291074$

O $3.017639108-0.614150676-1.851137312$

O $3.8795772541 .598652812-0.984726560$

S -3.4956473140 .1147890321 .032541343$

$\begin{array}{llll}\text { O } & -3.064683817 & -0.438241570 & 2.322036161\end{array}$

$\begin{array}{llll}\text { O } & -4.314886694 & 1.323451277 & 1.007668850\end{array}$

C $-4.466571180-1.2212347720 .172038277$

F $-4.715144163-0.863882870-1.092548607$

F $-3.758501119-2.3580132120 .171977979$

F $-5.619611498-1.4204711010 .811985804$

C $4.456517281-0.6186844560 .413702044$

F $4.601284725 \quad 0.064291614 \quad 1.555200468$

F $3.957692663-1.8297530090 .689615470$

F $5.645993213-0.753984746-0.174841247$

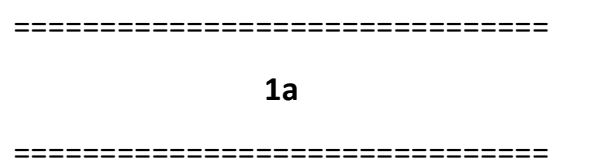

Bi $\quad-3.352006920 \quad 5.035685318 \quad 8.701922248$

C $-3.862679720 \quad 2.961887143 \quad 9.093479934$

C $-2.932317002 \quad 1.963675590 \quad 8.781971064$

C -5.1221911992 .6558846569 .628088991$

C $-3.2790290710 .630810943 \quad 9.016394808$

C -5.4495199421 .3165468559 .853047823$

C -4.5302694360 .3099597829 .547479317$

H $\quad-1.957853169 \quad 2.209591154 \quad 8.372388164$

$\begin{array}{llll}\text { H } & -5.845327000 & 3.429922760 & 9.874270729\end{array}$ 
H $\quad-2.566876374-0.154459371 \quad 8.782166851$

H $\quad-6.420869595 \quad 1.063981278 \quad 10.267036210$

H $\quad-4.790465497 \quad-0.728975378 \quad 9.725026558$

C $-4.216955035 \quad 5.681626015 \quad 6.812040102$

C $-5.0834626224 .757599063 \quad 6.215082172$

C $-3.939375691 \quad 6.930372248 \quad 6.247514586$

C $-5.693164755 \quad 5.1078140815 .008127296$

C $-4.5610282627 .254175483 \quad 5.039977297$

C $\quad-5.431965072 \quad 6.349960292 \quad 4.425968910$

H $\quad \begin{array}{llll}-5.280751226 & 3.785444104 & 6.659122832\end{array}$

H $\quad-3.240550223 \quad 7.6198481716 .705399512$

H $\quad-6.364162580 \quad 4.4048401854 .524478094$

H $\quad-4.354188094 \quad 8.214444747 \quad 4.577667199$

H $\quad-5.904583294 \quad 6.613124905 \quad 3.484723952$

C $-3.457897466 \quad 6.190760564 \quad 10.540120515$

C $-3.836318051 \quad 5.47656488911 .683806939$

C -3.209788291 7.56707866210 .561467868

C -3.9757140216 .17483533912 .885580519$

C $-3.355562136 \quad 8.241356276 \quad 11.775278505$

C $\quad-3.738595467 \quad 7.550481482 \quad 12.929068955$

H $-4.0159743324 .405353643 \quad 11.656908098$

H $\quad-2.885198873 \quad 8.1002082339 .675571321$

H $\quad-4.265989058 \quad 5.638723731 \quad 13.783818566$

H $\quad-3.163971575 \quad 9.309163840 \quad 11.816377681$

H $\quad-3.848386408 \quad 8.086530598 \quad 13.866562673$

O

S $\quad-0.482203073 \quad 5.911278493 \quad 7.889763507$

O $0.258614982 \quad 5.696612127 \quad 6.665762493$

O

C $0.704904840 \quad 5.995903614 \quad 9.337145225$

F $\quad-0.0182046696 .085389753 \quad 10.455130153$

F $1.448733275 \quad 4.9005506789 .357260065$

F $1.460294994 \quad 7.0746715439 .192686199$

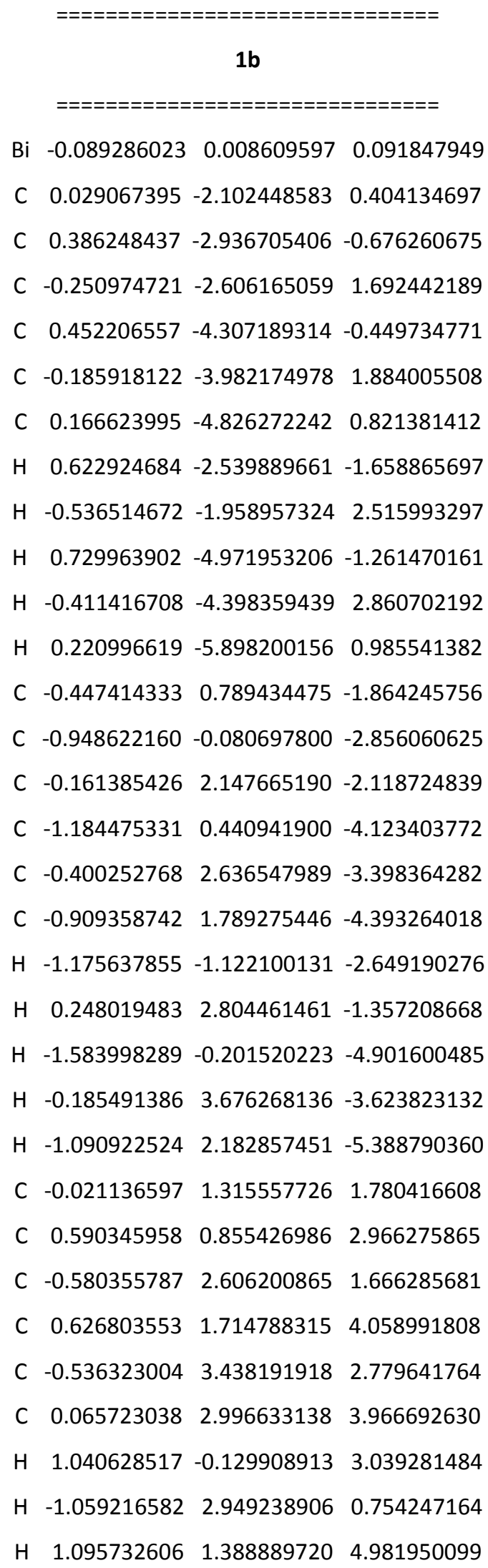


H $\quad-0.972785903 \quad 4.430409578 \quad 2.724711503$

H $\quad 0.100063294 \quad 3.658436552 \quad 4.826790523$

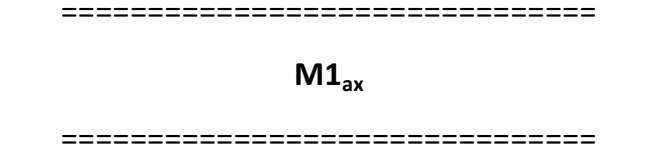

Bi $\quad 0.209116248 \quad 0.109578914 \quad 0.341468607$

C $-0.108455640-1.772363186 \quad 1.429928647$

C $0.700063252-2.873738229 \quad 1.160782387$

C $-1.042944743-1.764946250 \quad 2.464260650$

C $0.540895357-4.0155453191 .947252533$

C $-1.195554523-2.922741643 \quad 3.233710339$

C $-0.406382569 \quad-4.043697710 \quad 2.971991337$

H $1.433087987 \quad-2.849967200 \quad 0.366232336$

H $\quad-1.637021900 \quad-0.8831613682 .687792822$

H $1.159736696-4.8852738491 .747926036$

H $\quad-1.924497190 \quad-2.9392898924 .038852922$

H $\quad-0.525185900 \quad-4.940484342 \quad 3.573428349$

C $0.203197725 \quad 1.505330548-1.378773436$

C $-0.115085240 \quad 1.112749061-2.677449737$

C $0.4717818072 .833594325-1.048939071$

C $-0.173825205 \quad 2.095780466-3.670241563$

C $0.4137622293 .799064233-2.058707399$

C $0.0861478993 .433154814-3.364632854$

H $\quad-0.3057439450 .079665052-2.937089487$

H $\quad 0.756216573 \quad 3.122717835 \quad-0.043660279$

H $\quad-0.417612844 \quad 1.800146854$-4.686809131

H $0.637681743 \quad 4.833934442-1.817178029$

H $0.042660060 \quad 4.187034318$-4.145357090

C $0.023799375 \quad 1.543646422 \quad 2.025106870$

C $0.990359291 \quad 1.486569223 \quad 3.037032363$

C $-1.026476370 \quad 2.4637255592 .113260352$

C $0.9023443112 .350961845 \quad 4.130356670$

C $-1.112239783 \quad 3.323257307 \quad 3.211825367$

C -0.1478833693 .2666020614 .220152622$

H $\quad 1.816028561 \quad 0.7838055512 .972886601$
H $\quad-1.777281557 \quad 2.533526512 \quad 1.329757910$

H $1.659704010 \quad 2.309516447 \quad 4.908045356$

H -1.9283801844 .0376264053 .275959096$

H $-0.212459254 \quad 3.937852657 \quad 5.071616101$

O $2.287787958 \quad-0.228667193 \quad 0.739734525$

O $0.662288396-1.435676220-1.447991106$

S 3.5512359450 .6022206860 .335080109

O $3.192440086 \quad 1.987504251 \quad 0.026545157$

O $4.640811717 \quad 0.302403316 \quad 1.253787403$

S $0.082402456-2.558408286-2.288388705$

O $-0.588302180-3.601471506-1.502297991$

O $-0.628722624-2.044619943-3.474150136$

C $1.670397483-3.310194579-2.900455406$

F $2.390391627-2.397537422-3.560805051$

F $2.385640004-3.752049628-1.853157420$

F $1.413469902-4.339117119-3.713122313$

C $3.949898980-0.233808291-1.288121746$

F $3.984893136-1.556976370-1.118578774$

F 3.0162002720 .074830534 -2.191293572

F $5.140335168 \quad 0.190127213-1.718877543$

S $\quad-2.7423066490 .0088100370 .015800399$

C $-3.213430723 \quad 1.279110958$-1.208029549

H $-2.687050800 \quad 1.111833671$-2.148759811

H $\quad-4.294325924 \quad 1.251226629$-1.359248246

H $\quad-2.9196711912 .252375174 \quad-0.810265638$

C $-3.003780516-1.520038128-0.957287583$

H $\quad-2.576811851-1.414732403$-1.956441810

H $\quad-2.488765370 \quad-2.334592181-0.445787099$

H $\quad-4.074146399$-1.726635074 -1.014587581

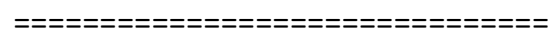

S1

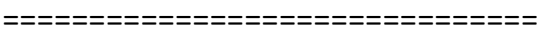

Bi $\quad-0.012964720 \quad-0.009683030 \quad 0.091939414$

C $-0.035092000-2.115230937-0.693671107$

C $0.865345105-2.468781369-1.698971566$ 
C $-0.967092611-3.026460905-0.197934430$

C $0.832139641-3.769464196-2.210766937$

C $-0.994636673-4.322672278-0.723244694$

C $-0.095814453-4.693265324-1.725365259$

H $1.588316318-1.754420116-2.082794169$

H $\quad-1.660672711-2.7368834440 .585150562$

H 1.534694587 -4.057063796 -2.989519547

H $-1.719112792-5.040792395$-0.346325014

H $\quad-0.118153739-5.703034213-2.128703331$

C $-0.4209325370 .851984577-1.945872891$

C $-1.2238233710 .123703533-2.829853635$

C $0.0795770392 .097743774-2.333238033$

C $-1.5160884650 .632348401-4.097020046$

C $-0.2067607632 .602093820-3.605618543$

C $-1.0040607341 .871889750-4.486997513$

H $-1.629914942-0.839490859-2.533536566$

H $0.710991858 \quad 2.670999389-1.663256205$

H $\quad-2.143956573 \quad 0.062394867$-4.778127743

H 0.1991051913 .565422324 -3.905591996

H $\quad-1.226604756 \quad 2.266728691-5.475810355$

C $0.2429425772 .018049092 \quad 1.026046899$

C $1.417257853 \quad 2.291406831 \quad 1.724729927$

C $\quad-0.748206167 \quad 2.984565028 \quad 0.870564917$

C $1.599167995 \quad 3.5598306732 .280375305$

C $-0.562340964 \quad 4.248753505 \quad 1.439044085$

C $0.608098771 \quad 4.534312140 \quad 2.143340367$

H $2.186335682 \quad 1.532410631 \quad 1.827151987$

H $\quad-1.656115037 \quad 2.777429471 \quad 0.311056210$

H $2.517052288 \quad 3.785094594 \quad 2.817892900$

H $\quad-1.335419395 \quad 5.005343794 \quad 1.330493645$

H $0.750333776 \quad 5.518697266 \quad 2.582990199$

O $2.224702663-0.079779566-0.217161297$

O $-2.284018401-0.0890850580 .198004775$

S $3.1904512250 .623081453-1.173268127$

O $3.375741634-0.117482375-2.426027164$
O $3.015250428 \quad 2.077811301$-1.230393999

S $\quad-3.3703953530 .5195617551 .083150235$

O

$\begin{array}{lllll}\text { O } & -4.298787398 & 1.362735026 & 0.326701976\end{array}$

C $-4.315917478-1.030078601 \quad 1.489111268$

F $\quad-4.800284777 \quad-1.5992714680 .378165249$

F $-3.518905113-1.918914752 \quad 2.109000579$

F $\quad \begin{array}{llll}-5.339447804 & -0.740251528 & 2.305516199\end{array}$

C $4.747955687 \quad 0.373252601 \quad 0.188313472$

F $4.6354085650 .953599134 \quad 1.017648527$

F 5.007423788 -0.927776684 0.017298704

F $5.7832918220 .935275842-0.833079518$

S $0.326622118-1.167476113 \quad 2.453920730$

C $-0.2356834330 .036443702 \quad 3.728385203$

H $\quad-0.617738040 \quad 0.929288369 \quad 3.228306810$

H $0.632988158 \quad 0.342438718 \quad 4.323450432$

C $-1.332737372-0.547097106 \quad 4.623689031$

H $-0.933879556-1.4004904975 .188335585$

H $-2.133386081 \quad-0.9304509263 .985850347$

C $-1.899277097 \quad 0.511179319 \quad 5.577151173$

H -2.3624612101 .3207585625 .003623061$

H $\quad-2.662384564 \quad 0.084555417 \quad 6.239106245$

H $\quad-1.114041831 \quad 0.946047723 \quad 6.209118788$

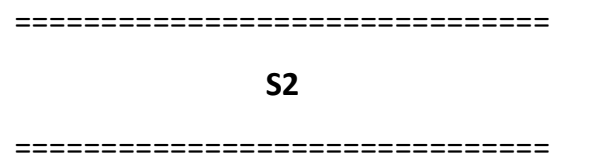

Bi $\quad-0.228666083 \quad 0.386848207$-0.201709474

C $0.022747462-1.793904373-0.417041794$

C $1.028760435-2.276160156-1.249453088$

C $-0.860079804-2.6427166730 .244746292$

C $1.151665816-3.661011569-1.409423506$

C $-0.723596474 \quad-4.020793348 \quad 0.075260301$

C $0.282455495-4.530645419-0.749417321$

H $\quad 1.729116474-1.612296886$-1.746729873

H $\quad-1.627599610 \quad-2.2441450310 .896610646$ 

H $1.943824212-4.051652743-2.043493756$
H $\quad-1.395932520 \quad-4.6929486720 .602888827$
H 0.392644168 -5.605576882 0.870397407
C $0.068717983 \quad 1.091568819-2.276187967$
C $-0.698152860 \quad 0.577291399 \quad-3.326566812$
C $\quad 1.074500085 \quad 2.027418492-2.541933314$
C $-0.4579892690 .994993905-4.638700813$
C $1.311879650 \quad 2.442754785$-3.853707364
C $0.547904095 \quad 1.927089857-4.902721717$
H -1.478917553 -0.155730932 -3.134110239
H $1.6917129982 .417328284-1.737381858$
H $\quad-1.0516014840 .586704837-5.453287410$
H $2.106346358 \quad 3.156986825$-4.053809166
H $\quad 0.741778056 \quad 2.244038268$-5.924542894
C $0.044693530 \quad 2.286778721 \quad 0.901251807$
C $0.9658459362 .429972657 \quad 1.937006359$
C $-\begin{array}{llll}0.723686688 & 3.359700759 & 0.451935849\end{array}$
C $1.114593030 \quad 3.685821466 \quad 2.529685844$
C $-0.5613672854 .612798785 \quad 1.053672692$
C $0.356784213 \quad 4.7750599532 .091959076$
H $\quad 1.556170991 \quad 1.593689488 \quad 2.287497158$
H $\quad-1.448536655 \quad 3.228467719$-0.347306872
H $1.828578375 \quad 3.8038754483 .340594382$
H $\quad-1.155342673 \quad 5.455458136 \quad 0.707931494$
H $\quad 0.482420728 \quad 5.748392238 \quad 2.560106851$
$\begin{array}{lllll}\text { O } & 2.093885217 & 0.481020198 & 0.033386497\end{array}$
O
S $3.476414661 \quad 0.401155524 \quad-0.586137841$
O $3.511633885-0.349253701-1.854699681$
O $4.183702761 \quad 1.688983706-0.540604934$
S $0.767956353-1.0633860003 .084932443$
O $1.473601701-2.147047464 \quad 2.389075013$
O $1.563192614-0.1487431203 .922562461$
C $-0.345096377-1.9401108224 .296251022$
F $-1.039089282-1.0649341715 .050347691$
F $-1.236669810-2.7228818953 .648527849$
F $0.368875862-2.722217704 \quad 5.121724538$
C $4.301690286-0.6962938010 .671879566$
F $4.114463290-0.2123130621 .903603639$
F $3.805404036-1.934549156 \quad 0.598472931$
F $5.627503706 \quad-0.750615898 \quad 0.431125038$
S $-2.7896053110 .479051302-0.188667227$
C $\quad-3.133329128 \quad 0.816642840 \quad 1.604797605$
H $\quad-3.960312880 \quad 1.536040260 \quad 1.612034330$
H $\quad-2.265525322 \quad 1.3134481792 .047740571$
C $-3.491557164-0.432735028 \quad 2.409813384$
H $\quad-2.643828889-1.120351167 \quad 2.389902027$
H $-4.342410582-0.9421951611 .939755758$
C $-3.811140501-0.0877446783 .868866732$
H $\quad-4.6670201720 .5951158563 .943857946$
H $\quad-4.045724519$-0.991269921 4.442370350
H $\quad-2.948884918 \quad 0.3862706894 .346892214$

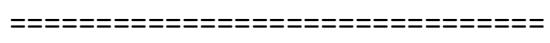
S3

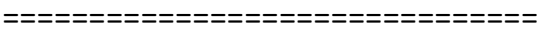
S $-6.605327147-1.808657138-9.110073297$
C $-6.441447717-0.573358532-10.483359051$
H $\quad-5.7599853150 .224030004-10.165719031$
H $\quad-6.004747153$-1.069051378 -11.355841834
C $-7.8102097160 .009557536-10.841487641$
H $\quad-8.2445749610 .494177403$-9.959061170
H $\quad$-8.487694489 -0.806132213 -11.120149724
C $-7.697507056 \quad 1.014993063-11.993893508$
H $\quad$-7.287260842 0.540516562 -12.892637846
H $\quad-7.039906449 \quad 1.850705469-11.728631637$
H $\quad$-8.678024548 1.428590929 -12.250997968
Bi -4.190036317 -2.659394249 -9.109511894
C $-4.557234719-4.398580789-7.802989593$
C $-3.494782872-5.130550134-7.263776289$
C $\quad-5.884993090-4.734405624-7.520715661$ 
C $-3.779483943-6.210486157-6.425438052$

C $-6.149795113-5.820811865-6.683702587$

C $-5.099498452-6.555407495-6.134063619$

H $\quad-2.469170124$-4.885911598 -7.506298186

H $\quad-6.713013000$-4.175989485 -7.945455101

H $\quad-2.958476360-6.784718935$-6.005651261

H $\quad-7.179827004-6.088293672-6.466175025$

H $\quad$-5.309506660 -7.398875954 -5.482426515

C $-4.061504035-2.983284653-11.264600005$

C $-4.781715374-4.036761803-11.828832884$

C $-3.237960990-2.164781239-12.037774692$

C $-4.663463751-4.278660947$-13.197756966

C $-3.133532370-2.415066517-13.407016108$

C $-3.841194852-3.469283631-13.983360520$

H $\quad-5.419961534$-4.672344344 -11.220934771

H $\quad-2.647589111-1.371929215$-11.590529704

H $\quad-2.481554311-1.794598310$-14.014377169

H $\quad-3.744362230$-3.668038922 -15.046742096

C $-3.248633233-0.812320964-8.359800980$

C $-4.0601754710 .068201905-7.639585413$

C $-1.906448957-0.519411727-8.618954588$

C $-3.511702515 \quad 1.258171618-7.156153425$

C $-1.3797693240 .682598173-8.139128254$

C $-2.173984219 \quad 1.565139983-7.406368849$

H $\quad-5.107060102-0.160498025$-7.460459950

H -1.273579441 -1.195584133 -9.179685509

H $\quad$-4.134565409 1.942125196 -6.586601701

H $\quad-0.339441363 \quad 0.921111023$-8.341371910

H $\quad-1.750838978 \quad 2.493024400$-7.031835883

O

S $-0.969260291-3.840394016$-10.166619946

O $-1.397345081-4.983002701-10.972142537$

O $-0.410495643-2.659141931-10.842155405$

C $0.382685229-4.457727085$-9.049246259

F $0.822632009-3.466365709$-8.264933442
F $\quad-0.082069370-5.448368833-8.268362961$

F $1.402085983-4.925935775$-9.773698573

H $\quad-5.205749983$-5.106309853 -13.645697271

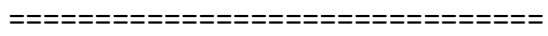

S -6.810062634 -1.602570956 -9.150871061

C $-6.712994123-0.390055738-10.564040902$

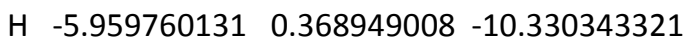

H $\quad-6.413020408$-0.923234276 -11.471290367

C $\quad-8.0916476880 .257205149$-10.744434940

H $\quad-8.3883702230 .750572984$-9.811550263

H $\quad-8.836291544$-0.521915955 -10.944559986

C $-8.070806504 \quad 1.273651530-11.892960938$

H $\quad$-7.802289855 0.797724500 -12.842406289

H $\quad$-7.352004308 2.078029081 -11.701767943

H $\quad-9.056925895 \quad 1.730162247$ - 12.015746151

Bi -4.535106481 -2.544717374 -9.184343874

C $-4.628923356-4.286040679-7.890938287$

C $-3.591355923-4.497853301-6.976790943$

C $-5.713580977-5.164974617-7.992526885$

C $-3.640786904-5.626122664-6.155365951$

C $-5.746220450-6.287072364-7.161928298$

C $-4.713180830-6.515883119-6.249874922$

H $\quad-2.761151513$-3.801685608

H $\quad-6.527849733$-4.984980119 -8.689156105

H $\quad-2.844278586$-5.804240587 -5.439640802

H $\quad-6.581605896 \quad-6.977375351 \quad-7.225976540$

H $\quad-4.746981917$-7.389207396 -5.605927154

C $-3.935417383-3.085426078-11.204668457$

C -4.126465297 -4.400237027 -11.644078694

C $-3.380902944-2.106175443-12.037618530$

C -3.750985018 -4.735970914 -12.946263903

C $-3.012120816-2.458718624-13.337483173$

C $-3.198753412-3.767734795-13.788379283$ 
$\begin{array}{cccc}\text { H } & -4.549729727 & -5.158337254 & -10.990412575 \\ \text { H } & -3.223335637 & -1.089133620 & -11.688518843 \\ \text { H } & -2.578390895 & -1.711267428 & -13.994614289 \\ \text { H } & -2.910198238 & -4.035075174 & -14.800222327 \\ \text { C } & -3.157077993 & -1.031220460 & -8.449948203 \\ \text { C } & -3.646391435 & 0.002187081 & -7.644653344 \\ \text { C } & -1.799177669 & -1.127887572 & -8.778777219 \\ \text { C } & -2.749902253 & 0.953760478 & -7.153473660 \\ \text { C } & -0.916173707 & -0.169711879 & -8.275712773 \\ \text { C } & -1.391346157 & 0.865110061 & -7.466457208 \\ \text { H } & -4.703575447 & 0.071604193 & -7.401106228 \\ \text { H } & -1.424548639 & -1.921689595 & -9.419872436 \\ \text { H } & -3.114480040 & 1.762133107 & -6.527226620 \\ \text { H } & 0.139839833 & -0.231691724 & -8.519894624 \\ \text { H } & -0.699649178 & 1.607259686 & -7.080010749 \\ \text { H } & -3.888918268 & -5.753034444 & -13.299900993 \\ & ==============================\end{array}$ Triflate ion (OTf ${ }^{-}$)

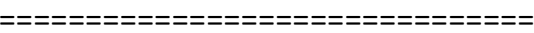

O $\quad-2.141248627 \quad-0.031297430 \quad 0.532937404$

S $-3.474213075-0.2163559841 .150488195$

O

O

C $-4.601426012-0.271979526-0.338765723$

F $-4.5333311410 .866570393-1.065357088$

F -4.286371740 -1.290396872 -1.170899596

F $\quad-5.896350710-0.4372100360 .014649603$

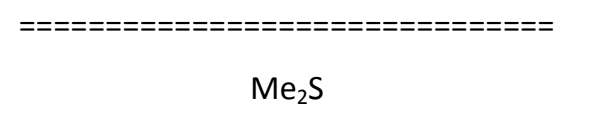

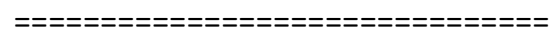

S $\quad 0.222944194 \quad 0.0001242730 .015658044$

C $-\begin{array}{llll}0.221442626 & 1.389637457 & 1.114297129\end{array}$

H $\quad-1.285984954 \quad 1.368375677 \quad 1.365420095$

H $\quad 0.372495764 \quad 1.371269757 \quad 2.033012619$

H $\quad-0.004307046 \quad 2.313535727 \quad 0.573089063$
C $-0.219113563-1.389883046 \quad 1.114703720$

H $-1.284904580-1.373764878 \quad 1.360830526$

H $\quad 0.005277217-2.3135515850 .576076146$

H $\quad 0.370406786-1.3668816912 .036160755$

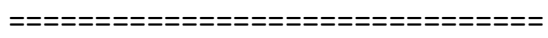

C $0.6648660630 .772949274 \quad-0.312775820$

O

C $-1.0489980712 .370318501-0.779143285$

C -1.947104091 $1.282638275-1.369664943$

C $-1.0259983820 .347320040-2.162714382$

C $0.014907571-0.287143889-1.233485647$

S $1.401313880-0.048548377 \quad 1.152373247$

C $\quad-0.104947042 \quad-0.349850375 \quad 2.183473371$

C $\quad-0.173674633 \quad-1.813275042 \quad 2.627847688$

C $-1.375490384-2.0453422793 .549051553$

C $-1.7752314803 .493726686-0.027918980$

O $1.101899524 \quad-0.824250276-1.975641446$

C $0.993348364-2.210034834-2.332899984$

O

C $-2.384796824-0.381917695$-3.985369241

O

C -3.8869982660 .9212029590 .041603484$

O

C $-2.6725632224 .986239707-1.703670238$

Bi $\quad 3.093362278 \quad 1.626228668 \quad 2.652285412$

C $2.824452720-0.0828742614 .029980191$

C $2.015616628 \quad 0.065352508 \quad 5.161485740$

C $3.398673360-1.3235960803 .731971564$

C $1.783386356-1.0323359735 .995505195$

C $3.164245700-2.4149251974 .571206018$

C $2.352953785-2.2721309995 .699794503$

C $4.7029484830 .977647668 \quad 1.282030179$

C $5.836126740 \quad 0.440612388 \quad 1.893998152$ 
C $4.5270479460 .967898752-0.101204775$

C $6.822912344-0.125883307 \quad 1.079635851$

C $5.5251865460 .393462833-0.892970921$

C $6.668097146-0.152277018-0.306568187$

C $1.6934942843 .149725618 \quad 3.436897462$

C $0.576405501 \quad 3.4947999412 .683565840$

C 1.9410990793 .6698594734 .707029769

C $-0.329114243 \quad 4.407543124 \quad 3.228860639$

C 1.0199172704 .5852544965 .227834530

C $-0.106827811 \quad 4.9548252204 .493207239$

C $-2.084887953 \quad 4.429828820-2.984785694$

C $-0.7121046534 .531599881-3.246052606$

C $-2.896042006 \quad 3.703821622-3.870647707$

C $-0.151653791 \quad 3.893219193-4.356472325$

C -2.341135872 $3.080410974-4.990067324$

C $-0.964320485 \quad 3.166358359-5.231975623$

C $-4.8700581640 .877876930-1.108741283$

C $-5.553777773 \quad 2.033302844-1.511064558$

C $-5.059148376-0.321468252-1.819288921$

C $-6.4372279321 .984449786-2.595678738$

C $-5.931689223-0.371146550-2.907785744$

C -6.6271620850 .783409138 -3.293856252

C $-3.142812726-1.583663823-4.502411084$

C $-3.221628359-2.776668184-3.770276960$

C $-3.830012840-1.482922957-5.722636592$

C $-3.986475014-3.848426099-4.248401325$

C $-4.592007774-2.551632376-6.200366635$

C $-4.675943192-3.739695673-5.461190902$

C $1.226745553-3.124443607-1.142048784$

C $0.276176199-4.084318240-0.755971633$

C $2.405466315-2.985249941-0.383714583$

C $0.501734196-4.9025273550 .363907231$

C $2.630519020-3.7937030290 .735414368$

C $1.678707930-4.7565138371 .111420665$

O $4.678318910 \quad 2.5472720893 .915550276$
S $5.288839507 \quad 2.059821485 \quad 5.252817063$

O $4.440899015 \quad 2.395690505 \quad 6.400030344$

C $6.752513855 \quad 3.211949946 \quad 5.296852083$

F $7.462258548 \quad 2.973052633 \quad 6.403741637$

F $7.522128814 \quad 3.000514983 \quad 4.225378631$

F $6.338152031 \quad 4.480661204 \quad 5.303263917$

O $5.8260119520 .699051730 \quad 5.148848105$

O $2.597102982 \quad 3.049727013 \quad 0.620077336$

S $2.233979601 \quad 3.920030552-0.549233851$

O $2.137830778 \quad 3.157713018-1.813171158$

O 1.1509564674 .881506244 -0.280119542

C $3.785073547 \quad 4.929793512-0.715029631$

F $3.6830535375 .784415942-1.739877662$

F $4.005957412 \quad 5.6211720650 .409405502$

F $4.828426163 \quad 4.113698879-0.931290865$

H $1.495127108 \quad 1.216341873 \quad-0.867830441$

H $\quad-0.427181690 \quad 2.794184671 \quad-1.571567831$

H $\quad-2.688646717 \quad 1.737542379-2.030993422$

H $\quad-0.4939369760 .943831756$-2.920901516

H $\quad-0.499448914$-1.059082167 0.647237111

H $\quad-0.059302500 \quad 0.328603780 \quad 3.042692769$

H $\quad-0.957850821 \quad-0.0519987421 .563561199$

H $0.748843922-2.084743116 \quad 3.151608448$

H $\quad-0.232097458$-2.459599410 1.743582916

H $-1.288744881-1.4464963184 .464991805$

H $-1.441950992-3.0993042593 .844415621$

H $\quad-2.316685598 \quad-1.774322540 \quad 3.054474427$

H $-2.122348172 \quad 3.0880907850 .928690436$

H $\quad-1.058112647 \quad 4.2992855210 .170304667$

H $0.021921371-2.412364881-2.805017733$

H $1.778893414-2.353054413$-3.086647240

H $\quad-3.0996370330 .439952840$-3.818773095

H $\quad-1.655620188$-0.023924611 -4.736818287

H $\quad-4.173693830 \quad 0.2065553180 .823907633$

H $\quad-3.877448842 \quad 1.9250356990 .484602324$ 
H $\quad-3.654528153 \quad 5.433190545$-1.894098843

H $\quad-2.013158983 \quad 5.761487272-1.286427544$

H $1.570715819 \quad 1.0255328825 .407836842$

H 4.025766965 -1.448510787 2.853374876

H $1.159212339-0.9137210636 .876989578$

H 3.613004045 -3.377262303 4.340075201

H 2.169793037 -3.123056451 6.349829350

H $5.9690065320 .445207522 \quad 2.970740212$

H $3.663420991 \quad 1.415010468$-0.572274260

H 7.711933655 -0.544662322 1.542910800

H 5.4014314930 .387602038 -1.972377194

H $7.438851253-0.596419268$-0.930743560

H $0.417138793 \quad 3.085741883 \quad 1.696476819$

H $2.810831912 \quad 3.397708679 \quad 5.291600426$

H $\quad-1.1992186124 .696935636 \quad 2.646887012$

H $1.200986055 \quad 5.010212693 \quad 6.211266096$

H $\quad-0.810219724 \quad 5.673380702 \quad 4.904982850$

H $\quad-0.067198452 \quad 5.057822717-2.545255179$

H $\quad-3.9596276803 .600025831-3.663009166$

H $0.922317816 \quad 3.945438789$-4.512508208

H $\quad-2.980496754 \quad 2.518048927-5.667778506$

H $\quad-0.529733482 \quad 2.668487160-6.095663715$

H $\quad-5.3634533642 .972908179-0.991154720$

H $-4.488147669-1.204652198$-1.538276209

H $\quad-6.966374674 \quad 2.886331722-2.902423668$

H $\quad-6.053003835-1.302178616-3.462941074$

H $\quad-7.3081989150 .747378004$-4.142294103

H $\quad-2.693705562-2.850734470-2.821401421$

H $\quad-3.776271610-0.555547892-6.295848644$

H -4.045840405 -4.769402350 -3.668063465

H $\quad$-5.123607692 -2.458763867 -7.147467209

H $\quad-5.272948323-4.572940609-5.829664878$

H $\quad-0.646721536-4.189040949-1.333803697$

H 3.129761009 -2.217626733 -0.663505204

H $\quad-0.245146902-5.6437500840 .656569506$
H 3.544229349 -3.670929200 1.320984638

H $\quad 1.850979527 \quad-5.383973754 \quad 1.988087215$

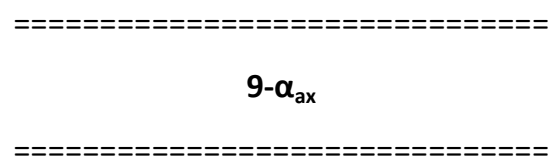

C $-1.975664754 \quad 1.483995466-0.888498952$

O

C $0.346112114 \quad 1.696912297$-1.525527192

C $0.1848157410 .593539736-2.566831647$

C $-0.710305817-0.489579485-1.950661322$

C $-2.0722546670 .136198997-1.648533514$

S $\quad-2.112247499 \quad 1.1624959040 .995797673$

C $-2.1843460592 .925752372 \quad 1.524238172$

C $-3.622377740 \quad 3.392125657 \quad 1.754724284$

C $-3.660946356 \quad 4.857380677 \quad 2.203792985$

C $1.2442133622 .834763566-1.985913990$

O $-2.840348811-0.815801172-0.934385094$

C $-4.257761071-0.641623275-0.974625605$

O

C $-0.335334974-2.829166799-2.487858889$

O

C -0.0872013020 .544848133 -4.969204953

O $2.447248218 \quad 2.245685500-2.409968605$

C $3.522982353 \quad 3.161080214-2.501931061$

Bi $-0.107368106-0.0627062262 .676155075$

C $0.806473812 \quad 1.8883322393 .084221138$

C $1.154790725 \quad 2.110971147 \quad 4.417143631$

C $1.080263442 \quad 2.8021944202 .074447586$

C $\quad \begin{array}{llll}1.793518931 & 3.311601662 & 4.736864271\end{array}$

C $1.7210886853 .994969426 \quad 2.414229566$

C $2.075643945 \quad 4.247456924 \quad 3.739464824$

C $-1.769402618-0.0717966224 .137547338$

C $-2.192310332 \quad 1.138008188 \quad 4.702229338$

C $-2.424674524-1.2642808504 .465929411$

C $-3.279598166 \quad 1.157010748 \quad 5.578822937$ 
C $-3.508883664-1.2381119195 .345857779$

C -3.942018033 -0.0304804165 .896750950$

C $-0.367301173-1.9998254651 .677070424$

C $-1.656816827-2.419736476 \quad 1.362822366$

C $0.748124469-2.8260956351 .539901619$

C $-1.833747493 \quad-3.7251994180 .893490696$

C $0.549150054-4.125097214 \quad 1.070432149$

C $-0.735664864-4.573797090 \quad 0.752654718$

C $4.763976450 \quad 2.447086616-2.977032610$

C $5.793817933 \quad 3.179553492-3.576688146$

C 4.9145235891 .069136015 -2.804561066

C $6.963227610 \quad 2.546222044 \quad-3.997692331$

C $6.0809487090 .434746609-3.232145826$

C $7.1093418841 .167401781-3.827054489$

C $1.3140618820 .893154459-5.414666752$

C $1.655884862 \quad 2.229036490-5.664985343$

C $2.293790355-0.093182928-5.546357098$

C $2.949564522 \quad 2.567770880-6.053506406$

C $3.592276870 \quad 0.244479480 \quad-5.935299699$

C 3.9225172881 .573102816 -6.192569999

C $1.084945461-2.964090883-2.990958188$

C $2.151386884-2.398676856-2.280366268$

C $1.350880380-3.626064078-4.197090434$

C $3.453928790-2.474656425-2.775937235$

C $2.655845332-3.719725062$-4.688090234

C $3.708932094-3.137203732-3.978798788$

C $-4.839003543-0.685074955-2.371900412$

C -4.268882056 -1.510869571 -3.349636539

C $-5.9507849930 .096974239-2.699121890$

C $-4.808323599-1.549872869-4.634367995$

C -6.4993009950 .046560828 -3.981998979

C $-5.927236668-0.776346439-4.952999049$

O $1.414862476-0.9264074673 .929596865$

S $1.203316701-1.7468598335 .234342902$

O $1.121080287-0.867574467 \quad 6.400599453$
O $0.210688317-2.807732205 \quad 5.052573437$

C $2.874535963-2.5716126515 .256996202$

F $2.970261217-3.321413623 \quad 6.358616468$

F $3.000157121-3.3543898414 .180959682$

F $3.842239046-1.6547539705 .260511923$

O $1.702228429-0.0767481870 .984685407$

S 3.1335247560 .3177594610 .645864606

O $3.718024373-0.589021095-0.349983282$

O $3.301235845 \quad 1.764320073 \quad 0.458646644$

C $4.072036051-0.1050231062 .198251339$

F $5.373696000 \quad 0.131572375 \quad 2.008592737$

F $3.6492990590 .620457863 \quad 3.239329042$

F $3.909972248-1.4111583472 .477765711$

H $-2.8892372342 .066907991-1.043347701$

H $\quad 0.757896393 \quad 1.253962587$-0.614797661

H $1.1758183090 .187196402-2.795005195$

H $\quad-0.251874733 \quad-0.844519543 \quad-1.017370568$

H $\quad-2.523976158 \quad 0.369083804-2.618981150$

H $\quad-1.591847338 \quad 3.020907610 \quad 2.435388917$

H $-1.670083322 \quad 3.5066282810 .752026567$

H $\quad-4.087579275 \quad 2.748871173 \quad 2.510976376$

H $\quad-4.204501348 \quad 3.2661870390 .832655789$

H $\quad-3.109983745 \quad 4.995947085 \quad 3.141254450$

H $\quad-4.690322302 \quad 5.191354744 \quad 2.367713282$

H $\quad-3.2099083795 .515534978 \quad 1.452491382$

H $0.750059293 \quad 3.380511882$-2.803588910

H $1.405131740 \quad 3.529231464$-1.145677623

H $\quad-4.643679704-1.470674751-0.370058994$

H $\quad-4.554561771 \quad 0.286945472 \quad-0.463395772$

H $\quad-0.379032703$-2.967003324 -1.402212476

H $\quad-0.972268828$-3.592070957 -2.948546749

H $\quad-0.223464592-0.538916868$-4.903377731

H $\quad-0.824763503 \quad 0.944075523 \quad-5.674304304$

H $3.271825046 \quad 3.976925125$-3.200958921

H $3.703324263 \quad 3.606092520-1.509235390$ 
H $0.976005247 \quad 1.366997027 \quad 5.189002588$

H $\quad 0.828673431 \quad 2.596304380 \quad 1.043207913$

H $2.0852457113 .500063685 \quad 5.766035317$

H $1.9640814994 .707937153 \quad 1.632133876$

H $2.587725520 \quad 5.170785718 \quad 3.995077241$

H $\quad-1.6704101572 .0660875584 .485499989$

H $\quad-2.079663846-2.2137289664 .070994359$

H $\quad-3.600504703 \quad 2.097477643 \quad 6.018497642$

H $-4.008246236-2.1672407165 .606192506$

H $\quad-4.785724750-0.0162820226 .580783866$

H $-2.510967942-1.7606144111 .463540635$

H $\quad 1.740238585$-2.475993688 1.794130676

H $\quad-2.830769647 \quad-4.0673446890 .631770271$

H $1.405426581-4.781764100 \quad 0.949100687$

H $\quad-0.878251417 \quad-5.5858602810 .384391200$

H $5.6787253294 .252352534-3.722340925$

H $4.1228147350 .500348302-2.337201093$

H $7.756843901 \quad 3.126378503$-4.462245679

H 6.184777784 -0.638935792 -3.095134543

H $8.017986672 \quad 0.670256823 \quad 4.157568543$

H $0.900745422 \quad 3.001705893 \quad-5.540086800$

H $2.048384751-1.127183795$-5.326595896

H $3.208296405 \quad 3.607236575$-6.239159470

H 4.350437958 -0.529937667 -6.008670894

H 4.9401278601 .840767901 -6.461973806

H $1.980090284-1.885338280-1.339563914$

H 0.529393736 -4.069123522 -4.756518844

H $4.256548132-2.012834190-2.212567309$

H 2.847890670 -4.237477635 -5.624404207

H 4.724394307 -3.199537916 -4.361679636

H $\quad$-3.370239673 -2.070119450 -3.109468743

H $\quad-6.3867823120 .754570740-1.949527985$

H $\quad-4.347891976$-2.178318651 -5.392059957

H $\quad$-7.361535359 0.661015338 -4.226737155

H $\quad-6.342862360 \quad-0.805039811-5.956569567$

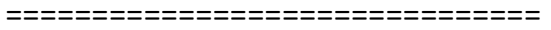

9- $\beta_{\text {eq }}$

C $-1.536372770-0.206770326-0.108995550$

O

C $\begin{array}{llll}0.873902887 & 0.309080108 & 2.106445912\end{array}$

C $-2.306494690 \quad 0.2423728292 .633662256$

C $-3.271996150 \quad 0.645120558 \quad 1.506634719$

C $-3.039990459-0.1769294860 .236036015$

S -1.190840498 -1.345105263 -1.508928659

C -1.156317213 -2.962059737 -0.634707300

C $-0.990957943-4.093918804-1.660036243$

C $-0.879587177-5.453588675-0.961778599$

C $0.160472722-0.1591563683 .137234532$

O

C $-4.957136081-0.114649554-1.263662259$

$\begin{array}{llll}\text { O } & -4.640241456 & 0.444527078 & 1.854752772\end{array}$

C $\quad \begin{array}{llll}-5.196314858 & 1.376048659 & 2.768704735\end{array}$

O $-2.605452455-1.0743486223 .065094902$

C $-2.929580205-1.2238876734 .443580443$

O $0.0269042150 .464579236 \quad 4.402824846$

C $0.485719172 \quad 1.8258873794 .478451420$

Bi 1.810346360 -0.639567901 -2.401219814

C $3.906222160-0.245823034-2.942365415$

C $4.439098412-0.551218650-4.200207868$

C $4.724767126 \quad 0.248183613-1.919360973$

C $5.800595474-0.337120793-4.433856351$

C $6.0824712220 .459725834-2.166141183$

C $6.6189485770 .169231290-3.422353643$

C 0.6788610720 .833886657 -3.617864049

C $1.339375704 \quad 1.248497546-4.777434805$

C $-0.5415883821 .384967820-3.228643069$

C $0.743998245 \quad 2.228159226-5.576259257$

C $-1.118554372 \quad 2.368960424-4.034337898$

C $-0.483288482 \quad 2.785103046-5.208325347$
\end{abstract}


C $2.156304450-2.143116192-0.805244319$

C $2.104801970-1.7635888220 .536574435$

C $2.518026055-3.434776985-1.202353544$

C $2.413914422-2.715581904 \quad 1.512779673$

C $2.819694568-4.373111527-0.209067232$

C $2.764832292-4.018145697 \quad 1.144105209$

C $-0.639452285 \quad 2.809477362 \quad 4.217560049$

C $-0.674058828 \quad 3.592842593 \quad 3.058191992$

C $-1.727098422 \quad 2.858683455 \quad 5.102176786$

C $-1.791930166 \quad 4.3813891712 .767500268$

C $-2.841270748 \quad 3.648516145 \quad 4.816449609$

C -2.881650152 $4.406131473 \quad 3.640022116$

C $-3.911226390-2.3633657094 .590013743$

C $-4.970674441-2.478848775 \quad 3.677030136$

C $-3.803793844-3.286255676 \quad 5.633503301$

C $-5.907035931-3.5038982813 .813751563$

C $-4.746409020-4.309805470 \quad 5.774139760$

C $-5.799430877-4.4216129534 .864104551$

C $-5.764533162 \quad 2.6207746772 .110144949$

C $\begin{array}{llll}-5.612271318 & 2.879742273 & 0.744185947\end{array}$

C $-6.460891878 \quad 3.542465865 \quad 2.905822107$

C $-6.150088841 \quad 4.0426816390 .185107551$

C $-6.997504580 \quad 4.702047693 \quad 2.348315123$

C $-6.844210844 \quad 4.9554053190 .980557790$

C $-4.832077859-1.454155352-1.969332803$

C $-5.347213078-2.624821816-1.390673337$

C -4.164373705 -1.541950949-3.205408848

C $-5.219775742-3.861736793-2.043523352$

C $-4.032371265-2.771472074-3.860262265$

C $-4.573201410-3.932628944-3.283121695$

O $1.520755406-2.363217834-3.742467129$

S $1.321881983-2.587862545-5.263674212$

O $1.762580240-1.424259953-6.042714128$

C $-0.548192548-2.600464638-5.366095250$

F -1.059050086 -1.495668522 -4.804065335
F $\quad-0.918786908-2.647401636-6.648547554$

F -1.033500646 -3.674692655 -4.730067608

O $1.766537753 \quad-3.926984179-5.635438977$

O $1.736107457 \quad 0.833687411 \quad-0.714970062$

S $1.4605938562 .187845265-0.068422125$

O $0.0858198292 .652402637 \quad-0.298472687$

O $1.981517875 \quad 2.219901181 \quad 1.303673444$

C $2.5482807863 .324613062-1.071713217$

F $2.3746830314 .582551033-0.659660625$

F $3.834972048 \quad 2.985536502-0.916867026$

F $2.227564033 \quad 3.230811886-2.368338695$

H $-1.2622175710 .796811861 \quad-0.455713050$

H $\quad-0.6610657691 .3353469591 .786437891$

H -2.3955395080 .9538933953 .458816085$

H $\quad-3.083342910 \quad 1.7025204641 .264384476$

H $-3.408691251-1.1965383810 .413266405$

H $\quad-0.338815609$-2.939131725 0.093483524

H $\quad$-2.105066844 -3.064549909 -0.087863199

H $\quad-0.092787271 \quad-3.914141247$-2.268405012

H $\quad-1.845497507$-4.090697129 -2.349940964

H $\quad-0.012589116 \quad-5.478005658$-0.285500964

H $\quad-0.759069171 \quad 6.260168542$-1.696363538

H -1.778912995 -5.670475792 -0.366106900

H 0.002860558 -1.231007766 3.293026642

H $1.166549616 \quad-0.0009263042 .724699036$

H $-5.619909495-0.191130589-0.390348285$

H $\quad-5.3787542600 .635930771$-1.948445002

H $\quad \begin{array}{llll}\text { H.989782698 } & 0.836880637 & 3.301567872\end{array}$

H $-4.459551795 \quad 1.678781708 \quad 3.527582622$

H $-2.019381274-1.3892454015 .041411193$

H $\quad-3.385907590 \quad-0.294567518 \quad 4.824811191$

H $\quad 0.868864719 \quad 1.943670779 \quad 5.499434272$

H $\quad \begin{array}{llll}1.314672838 & 1.991194811 & 3.778375525\end{array}$

H 3.817796183 -0.956925549 -4.992761417

H $4.315136000 \quad 0.479521172 \quad-0.940732752$ 
H $6.218645051-0.572052458-5.408966910$

H $6.718002766 \quad 0.849847347-1.375805408$

H $7.6764328690 .332308913-3.611179686$

H $2.2935842710 .830246625 \quad-5.076919334$

H $\quad-1.043781080 \quad 1.121859254$-2.309424741

H 1.2489495822 .549046568 -6.485187606

H $\quad-2.064434483 \quad 2.809485522-3.725277245$

H $\quad-0.940708915 \quad 3.548900415$-5.830755031

H $1.813490815-0.7622804090 .821085728$

H 2.559949439 -3.720460205 -2.249107237

H 2.368367778 -2.428057585 2.562477877

H $3.097232500-5.383281925-0.504410465$

H 2.998225045 -4.753883319 1.908765514

H $0.158385195 \quad 3.553328106 \quad 2.362641669$

H $\quad-1.708897301 \quad 2.243733331 \quad 5.999173785$

H $\quad-1.814932373 \quad 4.960746205 \quad 1.848487088$

H $\quad \begin{array}{llll}\text { H.683109191 } & 3.670267224 & 5.504337702\end{array}$

H $\quad-3.761878821 \quad 4.996481145 \quad 3.401684635$

H $\quad-5.036225465 \quad-1.761878298 \quad 2.863054977$

H $\quad-2.976378318$-3.208257460 6.336629727

H $\quad-6.723847250 \quad-3.589662114 \quad 3.100231238$

H $\quad$-4.651725351 $-5.024785005 \quad 6.587549914$

H $\quad-6.529271430-5.2213502954 .968175808$

H $\quad \begin{array}{llll}-5.062121865 & 2.182894010 & 0.121258788\end{array}$

H $\quad-6.583760155 \quad 3.346385344 \quad 3.970557764$

H $\quad-6.023589598 \quad 4.233066803 \quad 0.878539963$

H $\quad \begin{array}{llll}7.539441073 & 5.405065570 & 2.976994610\end{array}$

H $\quad-7.264121484 \quad 5.856709030 \quad 0.542004746$

H $\quad-5.851200062-2.566616185-0.423998381$

H $\quad-3.725430886$-0.642095335 -3.638257948

H $\quad-5.627197001$-4.764899912 -1.586538474

H $\quad-3.500219715-2.832410823$-4.808042926

H $\quad-4.474388041-4.892642854$-3.793943226

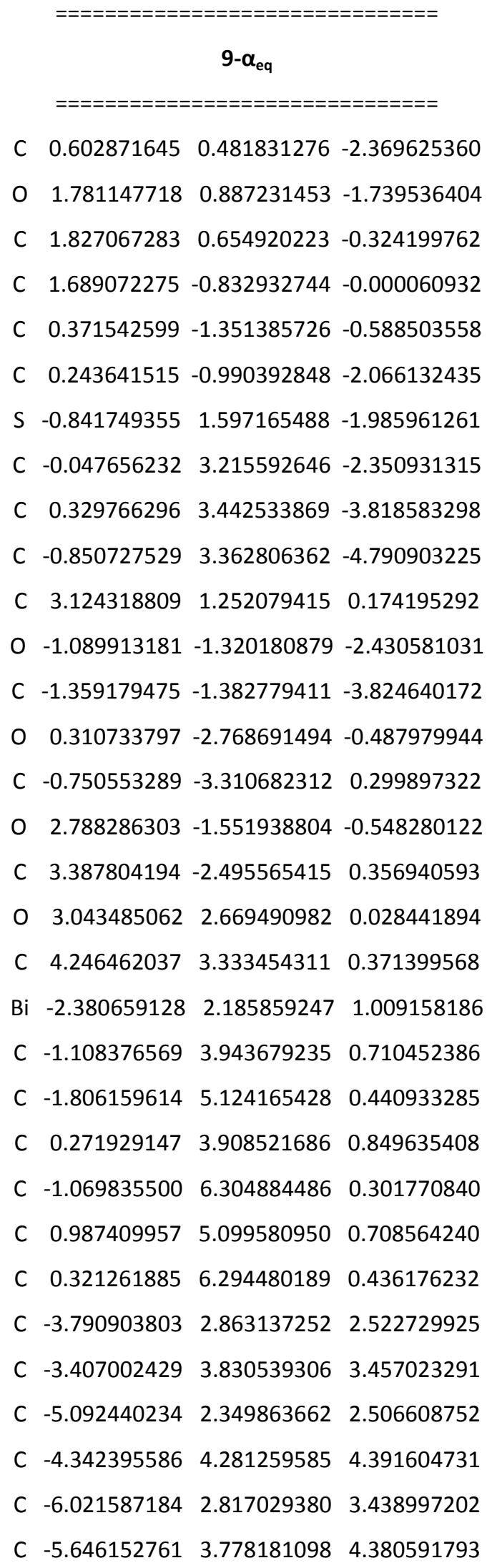


$\begin{array}{llll}\text { C } & -3.107999150 & 0.154850109 & 0.607591014\end{array}$

C $-3.248827510-0.6718676601 .725588110$

C $-3.580951885-0.206776741-0.651244263$

C $-3.879061738-1.9079360201 .566663720$

C $-4.197455822-1.453112839-0.790109652$

$\begin{array}{llll}\text { C } & -4.346901280 & -2.298638114 & 0.309719758\end{array}$

C 4.4986610673 .4250726841 .866973035

$\begin{array}{llll}\text { C } 5.788102897 & 3.691308470 & 2.342541773\end{array}$

C 3.4532842313 .2896629712 .784040477

C 6.0241193853 .8331869903 .710176108

C 3.6865766343 .4179280424 .153378576

C $4.9719163793 .695557615 \quad 4.620762711$

C $4.176041145-1.774165817 \quad 1.428486650$

C $5.376438244-1.131243526 \quad 1.091167311$

C $3.649940559-1.5907534722 .712163588$

C $6.016592518-0.2956919492 .004401008$

C $4.284621231-0.746138048 \quad 3.627843625$

C $5.462906522-0.0899996913 .272689398$

C $-0.289756725-3.592494596 \quad 1.712784926$

$\begin{array}{llll}\text { C } & -0.182341853 & -2.554918930 & 2.649543381\end{array}$

C $0.133427679-4.876484852 \quad 2.077176439$

C $0.357453664-2.7947128203 .914377127$

C $0.661303771-5.123155424 \quad 3.345680315$

C $0.780240432-4.0796796004 .265160802$

C $-0.633627942-2.510630960-4.530719415$

C $-0.249186019-3.659576546-3.828007339$

C $-0.354059360-2.421621012-5.898626120$

C $0.400982483-4.702284487-4.488660495$

C $0.283376862-3.470735299-6.562417780$

C $0.662741804-4.615187198-5.857788086$

o $-3.5033224952 .776782935-0.764448012$

S $-4.869915993 \quad 3.437013629-1.052273927$

O $-5.9468013852 .451852631-1.122886468$

O

C $-4.4595223623 .946357749-2.794441201$
F $\quad-5.492084373 \quad 4.598685418-3.334126616$

F $-3.3864539794 .754907952-2.787944373$

F $\quad-4.182471642 \quad 2.866856781-3.533151965$

$\begin{array}{lllll}\text { O } & -0.854701212 & 1.081814950 & 2.240851241\end{array}$

S $\quad 0.067420907 \quad 1.3881919943 .440491166$

$\begin{array}{llll}\text { O } & 1.248684916 & 0.527047833 & 3.398690961\end{array}$

$\begin{array}{llll}0 & 0.218789176 & 2.825291360 & 3.685509568\end{array}$

$\begin{array}{llll}\text { C } & -1.001022462 & 0.776593680 & 4.837121314\end{array}$

F $-2.084000682 \quad 1.554055703 \quad 4.955187824$

F $-1.414192762-0.481512205 \quad 4.600868331$

F $-0.3089771290 .795691265 \quad 5.977293890$

H $0.7962963900 .597385492-3.438986294$

H $\quad 0.984657573 \quad 1.1696154480 .157290268$

H $1.652456201-0.9338873661 .090425440$

H $-0.460610620-0.882192312-0.046051012$

H $\quad 0.972590802-1.607571952-2.607236338$

H $\quad-0.794958708 \quad 3.947803281-2.030917939$

H $0.8295101783 .305461481-1.706204706$

H $1.1194206512 .739431130-4.111179640$

H $\quad 0.790038957 \quad 4.438931007-3.873830122$

H $-1.2888108652 .359305873-4.803320744$

H $\quad-0.5413691303 .611956233-5.811517723$

H $-1.6478352434 .054171365-4.495676665$

H 3.2843613990 .9601298391 .219600070

H $3.9527285940 .851951186-0.423176275$

H $-2.445535641-1.523850859-3.888207506$

H $-1.147177756-0.420632338-4.315642187$

H $-1.609767173-2.6347802510 .290531689$

H $-1.055938365-4.240332178-0.194806929$

H $2.619008876-3.1467544630 .791427343$

H $4.043068353-3.107376533-0.271728483$

H $5.1076927072 .855027468-0.123754872$

H $4.157005480 \quad 4.343481415-0.051384543$

H $\quad-2.886352517 \quad 5.148388345 \quad 0.332511348$

H $\quad 0.8094208832 .992875791 \quad 1.035186515$ 
H $\quad-1.595364513 \quad 7.2314238210 .088889446$

H $2.064550994 \quad 5.0735541390 .817649762$

H $\quad 0.883372246 \quad 7.217530674 \quad 0.327522735$

H $\quad-2.391906444 \quad 4.219005963 \quad 3.473561080$

H $\quad \begin{array}{llll}5.396069195 & 1.613374411 & 1.766774971\end{array}$

H $\quad-4.051913971 \quad 5.027188872 \quad 5.125938472$

H $\quad \begin{array}{llll}7.037550350 & 2.433197186 & 3.423748342\end{array}$

H $\quad-6.3710484594 .1384213325 .104999089$

H $\quad-2.876088213 \quad-0.380344730 \quad 2.700197602$

H $\quad$-3.485360888 0.456384755 -1.500264155

H $\quad-3.984495073 \quad-2.565695013 \quad 2.424325490$

H $\quad-4.560838418$-1.756678222 -1.767286594

H $\quad-4.829001328$-3.264530579 0.188394139

H $6.611835543 \quad 3.789474827 \quad 1.637699332$

H $2.453811072 \quad 3.067478408 \quad 2.438963125$

H 7.0286581724 .0467860204 .066702186

H $2.855164779 \quad 3.2990867194 .841857803$

H $5.156743273 \quad 3.800825268 \quad 5.686638655$

H $5.784349831-1.2610522030 .090844803$

H $2.720728489-2.0818581912 .985544811$

H $6.935947626 \quad 0.213528605 \quad 1.726319798$

H 3.836919866 -0.576307236 4.602604557

H $5.936568563 \quad 0.597451172 \quad 3.967723289$

H $\quad-0.503841093-1.5525814932 .384476796$

H 0.057762085 -5.686699553 1.355179004

H 0.457287869 -1.976676540 4.618289896

H $0.987661810-6.125088844 \quad 3.612736418$

H $1.202545893-4.2642300425 .249384787$

H $\quad-0.424476635$-3.706878821 -2.757549761

H $\quad-0.631972543-1.523355432$-6.446760330

H 0.709654359 -5.582645449 -3.930831984

H 0.496054965 -3.389653721 -7.625140449

H $1.171170096-5.427734491-6.369885014$

\begin{abstract}
ニニニニニニニニニニニニニニニニニニニニニニニニニニニニニニニ 4- $\beta$

C $2.9199948050 .903220216-2.722973786$

O $1.818858927 \quad 1.758264966$-3.007619908

C 0.5850868341 .099275198 -3.257441844

C 0.2026100750 .246629412 -2.040592351

C $1.260246342-0.856187924-1.899799912$

C $2.630424692-0.204433426-1.670769603$

S $4.3338332661 .979463019-2.290611417$

H 3.2459129880 .384231565 -3.638431343

H 0.6597434630 .445671792 -4.144006672

H $\quad-0.798142952-0.176791819-2.184730179$

H 1.299064138 -1.437564828 -2.836107362

H $2.603191534 \quad 0.220953607$-0.664959194

C $3.8889431412 .605591097-0.615209027$

H $3.814820118 \quad 1.7621631790 .079460647$

H $4.781642025 \quad 3.172151137$-0.322627479

C $2.6370709243 .483574105-0.521626223$

H $\quad 1.767565774 \quad 2.891271592-0.811603795$

H $2.719826456 \quad 4.306196056-1.241301479$

C $2.448214471 \quad 4.0239941190 .901269413$

H $2.3391725123 .206515343 \quad 1.622005900$

H $1.541367230 \quad 4.6336096420 .970222393$

H $3.296916377 \quad 4.644740792 \quad 1.214825624$

C $-0.426807737 \quad 2.194576769$-3.558907351

H $\quad-0.588097765 \quad 2.800836397$-2.658304892

H $\quad-0.0300969512 .854542593 \quad-4.344720078$

O 3.622301048 -1.216112379 -1.783253907

C $4.659413837-1.192799551-0.796771726$

H $5.227846338-0.255945743-0.855663303$

H 5.328909772 -2.007570527 1.094904782

O $1.034070545-1.727280277-0.806015926$

C $-0.057033922-2.635898745-0.930503082$

H $\quad-0.296505893-2.825468413-1.986811556$
\end{abstract}


H $0.293291972-3.583224137-0.500418227$

O $0.272252109 \quad 1.031451732-0.861333015$

C $-0.926731478 \quad 1.415998101-0.204526236$

H $-1.6658715780 .607115021-0.260715681$

H $-1.377716240 \quad 2.298153285$-0.676602476

O

C -2.7323360772 .428481697 -4.110185389

H $-2.4490458623 .345514202-4.649689600$

H $\quad-3.4586413891 .889958518$-4.728762450

C $-3.3440530392 .771545414-2.766980108$

C $-3.3974960914 .087000812-2.297939148$

C $-3.838794693 \quad 1.740834998-1.955649115$

C $-3.9469714384 .374125544-1.044993088$

C $-4.381963445 \quad 2.020855034-0.704684094$

C $-4.442178619 \quad 3.341465020-0.247935855$

H $\quad-3.001932260 \quad 4.891913048$-2.912925920

H $\quad-3.765430171 \quad 0.712419142$-2.298270678

H $\quad-3.979383654 \quad 5.400409163$-0.690130563

H $\quad$-4.739109305 $1.209568112-0.078029375$

H $\quad-4.864942401 \quad 3.561296560 \quad 0.728481835$

C $-\begin{array}{llll}0.595392782 & 1.705027636 & 1.239132232\end{array}$

C $-1.354249765 \quad 2.6272668691 .965261500$

C $0.443003873 \quad 1.016754694 \quad 1.880073239$

C $-1.0920309512 .848091013 \quad 3.318832767$

C $0.707425069 \quad 1.240269337 \quad 3.230811439$

C $-0.0618068312 .154162087 \quad 3.955863486$

H $\quad-2.152378683 \quad 3.171951747 \quad 1.466482162$

H $\quad \begin{array}{llll}1.032764028 & 0.304684432 & 1.314074115\end{array}$

H $\quad-1.687108765 \quad 3.568322562 \quad 3.874205643$

H $\quad 1.516588557 \quad 0.696682822 \quad 3.710374684$

H $0.145093473 \quad 2.3302584915 .008069956$

C $-1.285064254-2.157741871-0.182441335$

C -1.158931796 -1.787021392 1.162208332

C $-2.534990008-2.050582027-0.801092333$

C $-2.255015005-1.2958683391 .868967118$
C $-3.640364340-1.571987160-0.090913618$

C -3.500559743 -1.185979252 1.243934365

H $-0.185288290-1.8490592161 .638358118$

H $\quad-2.643556380$-2.329392098 -1.846859807

H $\quad-2.130230023 \quad-0.9759530112 .899074571$

H $\quad-4.605471849$-1.490615873 0.583914403

H $\quad-4.354161699$-0.799184543 1.793437509

C $4.155793006-1.4163033840 .615462767$

C $3.319459045-2.5054020710 .897270207$

C $4.494674404-0.5375342921 .650638122$

C $2.835556930-2.7121039912 .187365998$

C $4.014424836-0.7424600042 .948568960$

C $3.182326304-1.830348113 \quad 3.218617861$

H $3.025905054 \quad-3.163441160 \quad 0.085884167$

H $5.140708350 \quad 0.313139667 \quad 1.442170359$

H 2.185209979 -3.559542459 2.393065128

H $4.286655721 \quad-0.050937222 \quad 3.742507221$

H $2.803264825 \quad-1.991489084 \quad 4.224721796$

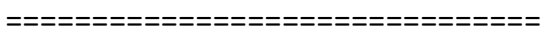

4- $\alpha$

C $1.973122747 \quad 1.4055949990 .072155961$

O $1.246177140 \quad 1.130846779-1.103087582$

C $0.987796812-0.259410196-1.346475949$

C $0.157055231-0.862641106-0.205550295$

C $0.960658123-0.6929067831 .086508625$

C $\quad \begin{array}{llll}1.284095308 & 0.785707657 & 1.302940939\end{array}$

S $3.7581167510 .854390098-0.030910532$

H $\quad \begin{array}{llll}1.971121200 & 2.493438668 & 0.169105969\end{array}$

H $1.938040710-0.802478612-1.389745522$

H 0.010266008 -1.930146919 -0.390384992

H $1.896178274-1.2558727150 .976664093$

H $0.333747206 \quad 1.3161258551 .430089049$

C 4.1007524201 .314688665 -1.783044460

H $5.177277048 \quad 1.136442170-1.886633239$ 
H $3.586917210 \quad 0.600280493-2.433045085$

C $3.7521065532 .751064411-2.198895192$

H $2.664395315 \quad 2.869874404-2.175389413$

H $4.047437136 \quad 2.861084887$-3.251793685

C $4.4246095293 .842248129-1.362915032$

H $\quad 4.122582714 \quad 3.779551505$-0.312899235

H $4.1580255994 .837034345-1.734880547$

H $5.516380396 \quad 3.751184419$-1.394990539

C $0.297406284-0.285163415-2.713605549$

H $\quad$-0.536289661 0.422081749 -2.682558546

H 1.0035708090 .059979506 -3.481223652

O $2.116879226 \quad 0.948474352 \quad 2.449844793$

C 1.4370077501 .4490715063 .600659715

H $2.173649661 \quad 1.3819782524 .409999669$

H $0.588593727 \quad 0.800408500 \quad 3.858916552$

O $0.213558687-1.1844094642 .199085126$

C $0.996673570-1.9136440003 .138925213$

H 1.906417785 -1.359483820 3.402904762

H 0.372161640 -1.976719372 4.038469892

O

C $-2.227450519-0.810581411-0.650549508$

H $\quad$-2.133746597 -0.839236228 -1.741671299

H $\quad-3.060458344-0.146246410-0.398588627$

O $-0.245445681-1.560621888-3.043558851$

C $0.618988023-2.432748768$-3.780547178

H $0.007011480-3.318148125$-3.981643123

H $0.880398464-1.973684265$-4.746364889

C $1.872016145-2.801489819-3.021105837$

C $3.115886365-2.287266014-3.399817025$

C $\quad 1.777810314-3.557093822-1.843925628$

C $4.245916133-2.505922610-2.608534738$

C $2.900189349-3.768019769-1.044616909$

C $4.136174976-3.238055216-1.426378111$

H $3.196320227-1.693602972$-4.307599896

H $0.812583479-3.950395306-1.533914528$
H $5.204369049-2.089966898$-2.906140882

H $2.807774391 \quad-4.332043114-0.122366905$

H 5.008454003 -3.388949951 0.797198693

C -2.501728703 -2.206638085 -0.135146814

C $-2.297172701-3.323233133-0.953179724$

C $-2.931095351-2.397505618 \quad 1.183930421$

C $-2.532210244-4.612439452-0.466671063$

C $-3.175411778-3.6811904991 .669126906$

C $-2.976662150-4.792891747 \quad 0.843703450$

H -1.938321642 -3.169741043 -1.967120140

H $\quad-3.051294764$-1.533276920 1.830885150

H $\quad-2.367828947$-5.473101257 -1.109855140

H $\quad-3.510108854 \quad-3.819769572 \quad 2.693355763$

H $-3.159300671 \quad-5.793588006 \quad 1.225522420$

C $1.353667384-3.3099991262 .668799356$

C $0.448621552-4.0415575231 .890874623$

C $2.566281769-3.898969828 \quad 3.039308402$

C $0.742730080-5.350109606 \quad 1.510289131$

C $2.862807206-5.2104564212 .660765334$

C $1.949150490-5.9413407391 .898216725$

H $\quad-0.480640163 \quad-3.576597548 \quad 1.585115056$

H 3.283522421 -3.328429095 3.625454325

H $0.026424643 \quad-5.9034716370 .909383160$

H $3.809097191 \quad-5.6571113782 .954085304$

H 2.180480594 -6.960349560 1.600307106

C $0.967165347 \quad 2.878701569 \quad 3.426189197$

C $\quad-0.295407170 \quad 3.281092524 \quad 3.872768208$

C $1.8013768323 .814870403 \quad 2.798627469$

C $-0.718950464 \quad 4.602827357 \quad 3.707309216$

C $1.377005393 \quad 5.132281948 \quad 2.624620313$

C $0.1153908965 .530062753 \quad 3.080208468$

H $\quad-0.9545885992 .553864606 \quad 4.343483276$

H $2.774298239 \quad 3.4918619312 .435792532$

H -1.7051363734 .9025946424 .054020955$

H $2.027827229 \quad 5.849964137 \quad 2.130603349$ 
H $\quad-0.217176554 \quad 6.555875306 \quad 2.940922124$

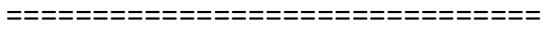

$10-\beta$

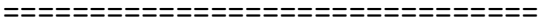

C $0.161467532-0.216904143-0.037326796$

O

C $-1.100024192-1.282916521 \quad 1.676843326$

C $-0.041501871 \quad-0.895656445 \quad 2.735186599$

C $\quad 1.325017187-1.1410121592 .059295744$

C $1.435262713 \quad 0.2117991960 .838429859$

S $0.110526581 \quad 1.292116758-1.111318465$

C $0.334606174 \quad 2.666588500 \quad 0.111742191$

C $0.008318254 \quad 4.028563813 \quad-0.504046794$

C $0.216842680 \quad 5.1498880810 .521069956$

C $-2.534344185-1.495916170 \quad 2.133114813$

O $2.465815959-0.574950703-0.056916923$

C $3.801367249-0.1208573910 .259638100$

O $2.454945237-0.8299765762 .851788893$

C $2.634473494-1.5817050934 .057172502$

O

C $\quad-0.779069533 \quad 0.862721895 \quad 4.246779468$

O

C $-4.507016475-0.515438042 \quad 2.980517778$

Bi $\quad-2.707771434 \quad 1.166114465$-1.763743466

C $-3.2112013552 .632093451-0.215192333$

C $-2.857296864 \quad 2.486213334 \quad 1.122283136$

C $-3.812618623 \quad 3.784632820-0.727547848$

C $-3.109978007 \quad 3.562579831 \quad 1.979435151$

C $\quad-4.055375405 \quad 4.842554428 \quad 0.151320733$

C $-3.698322441 \quad 4.732558672 \quad 1.497704757$

C -2.2902720142 .089919733 -3.696864021

C $-2.987784951 \quad 1.580196855-4.799369418$

C $-1.3584164373 .120216209-3.848393774$

C -2.7410086562 .114091268 -6.065206427

C $-1.126758040 \quad 3.649739590 \quad-5.119757750$
C $-1.813887754 \quad 3.145462229-6.225025058$

C $-2.373827536-0.977183123-2.109224697$

C $-3.103852292-1.989869107-1.490117530$

C $-1.368120001-1.247626168$-3.040807014

C $-2.797814181-3.315498953-1.807547719$

C $-1.075054956-2.581383728$-3.341335892

C $-1.784221448-3.610775998-2.721208966$

C $\quad \begin{array}{llll}-5.121698917 & 0.810938258 & 3.336003129\end{array}$

C $\quad \begin{array}{llll}5.999725136 & 1.443034049 & 2.450319170\end{array}$

C $-4.799515740 \quad 1.4419717024 .544030035$

C -6.5381498812 .6920563962 .757141053$

C $\quad-5.331665591 \quad 2.694084255 \quad 4.852036914$

C $-6.199177216 \quad 3.323086162 \quad 3.954395099$

C $-0.589239278 \quad 2.3493040354 .423212490$

C $\begin{array}{llll}-1.482318287 & 3.071779088 & 5.223864462\end{array}$

C $0.4815691893 .024002658 \quad 3.823839073$

C $-1.313479348 \quad 4.443935654 \quad 5.418752401$

C $0.649881506 \quad 4.3959998554 .017403108$

C $-0.2465943615 .112566857 \quad 4.813082363$

C $2.144241313-0.8219884195 .271672230$

C $2.393760521 \quad 0.552462578 \quad 5.373815200$

C $1.457548439-1.4665222396 .305182378$

C $1.959360555 \quad 1.2721730926 .484459037$

C $1.025871707-0.7481388927 .424194675$

C $1.273624167 \quad 0.622554470 \quad 7.514465705$

C $3.886331937 \quad 1.382523498 \quad 0.382682145$

C $3.916277518 \quad 1.9971810681 .641213098$

C 3.8608584372 .186507848 -0.765385798

C $3.9276162173 .389987762 \quad 1.753055792$

C $3.8775966903 .577516745-0.658621102$

C 3.9120616094 .1817475520 .602496167

H $0.246521795-1.045944708$-0.753381405

H $\quad-0.804039837 \quad-2.2501181891 .235964742$

H $\quad-0.148005307$-1.523918265 3.630479316

H $1.375542495 \quad-2.192428325 \quad 1.732718121$ 
H $1.593084463 \quad 0.780085076 \quad 1.265373429$

H $\quad-0.2793589892 .443187404 \quad 0.985205407$

H $\quad 1.388042242 \quad 2.638569784 \quad 0.395514338$

H -1.0306591564 .055707266 -0.846081691

H $0.655891080 \quad 4.197176277-1.374012558$

H $\quad-0.428900352 \quad 5.007518474 \quad 1.392005310$

H $\quad-0.018309968 \quad 6.1215688220 .075456792$

H $\quad 1.254470717 \quad 5.174928909 \quad 0.867198525$

H $\quad$-3.106172544 -1.9063384291 .288480103$

H $\quad-2.516630268$-2.270457500 2.920657200

H $4.151821138-0.6059464621 .174448941$

H $4.401750124-0.474847999-0.582792128$

H $2.147783772 \quad-2.564673374 \quad 3.985167594$

H 3.713963508 -1.759308996 4.132766884

H $\quad-1.844915024 \quad 0.621952674 \quad 4.176686630$

H $\quad-0.347703178 \quad 0.324466608 \quad 5.101819335$

H $\quad-5.050225291 \quad-0.979036702 \quad 2.146406373$

H $\quad$-4.534925596 -1.205471205 3.838880536

H $\quad-2.428160950 \quad 1.569785478 \quad 1.508050094$

H $\quad$-4.100960868 3.866831849 -1.771643131

H $\quad-2.854917520 \quad 3.469234782 \quad 3.028629295$

H $\quad-4.527522314 \quad 5.746358252 \quad 0.223039027$

H $\quad \begin{array}{llll}3.889093219 & 5.557605070 & 2.177097052\end{array}$

H $\quad-3.725516340 \quad 0.793290753 \quad-4.680336534$

H $\quad-0.814303267 \quad 3.520082803-3.000714585$

H $\quad-3.278731133 \quad 1.723197193$-6.923964544

H $\quad-0.407713024 \quad 4.454259541$-5.243392986

H $-1.627462273 \quad 3.557408083$-7.212442931

H $\quad-3.901636981-1.763152055-0.794824254$

H $\quad-0.820522978-0.451420896-3.538514281$

H $\quad-3.364078678$-4.115694494 -1.339864800

H $\quad-0.295766212-2.807480220-4.062904518$

H $\quad$-1.553905133 -4.645070536 -2.958496337

H $\quad-6.2552427020 .9529722201 .519791780$

H $\quad-4.137039761 \quad 0.943227447 \quad 5.247789086$
H $\quad-7.210690008 \quad 3.172462124 \quad 2.052556915$

H $\quad-5.078712088 \quad 3.176241778 \quad 5.792514204$

H $\quad-6.617739503 \quad 4.2966281174 .195262159$

H $\quad-2.322606793 \quad 2.561231221 \quad 5.687368227$

H $\quad 1.181574715 \quad 2.470781067 \quad 3.207610925$

H $-2.018263457 \quad 4.992194843 \quad 6.038631725$

H $1.4833463994 .906011768 \quad 3.540594347$

H $\quad-0.116557184 \quad 6.181305034 \quad 4.960389864$

H $2.899003738 \quad 1.0624306714 .560521138$

H $1.255293567 \quad-2.5335841906 .237482324$

H $2.135758695 \quad 2.342744650 \quad 6.536502716$

H 0.489936145 -1.257842852 8.220189150

H $0.929414481 \quad 1.184178353 \quad 8.378279796$

H $3.918816371 \quad 1.373336968 \quad 2.529805130$

H $3.825770967 \quad 1.715388544$-1.744482239

H $3.952898752 \quad 3.854773574 \quad 2.735052414$

H $3.871857345 \quad 4.191315961$-1.555258388

H $3.933187410 \quad 5.265009378 \quad 0.685556760$

O

S $\quad-5.837910660 \quad 0.285278667$ - 1.637704790

O $-6.486072746-0.749699555-2.427037453$

C -7.117056395 1.589403725 -1.254089106

F $-6.5378401972 .609515832-0.607401480$

F $-7.669276004 \quad 2.030902085-2.378646046$

F $-8.048863402 \quad 1.051157527-0.466744604$

O

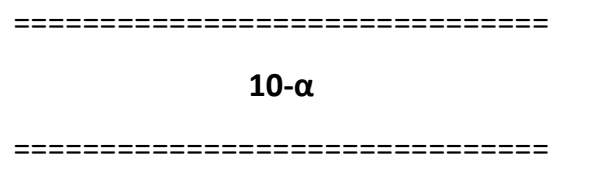

C $0.499139768 \quad 1.992410887 \quad 0.564035414$

$\begin{array}{llll}\text { O } & -0.695071747 & 1.345985227 & 0.799575307\end{array}$

C $-0.835943021 \quad 0.0490123730 .177883930$

C $0.255160788-0.9030719660 .655686614$

C $1.607173992-0.2751618250 .251684795$

C $\quad \begin{array}{llll}1.723656180 & 1.116635731 & 0.872984707\end{array}$ 
S $0.610758060 \quad 2.681337773-1.230522117$

C $0.9246711594 .481952107 \quad 0.920917238$

C $1.8785222635 .067244181-1.969944967$

C $3.3124519614 .539088943-1.853225080$

C $-2.251893984-0.3960536250 .460274018$

O $2.882762879 \quad 1.798418231 \quad 0.438795217$

C $3.956990777 \quad 1.836925513 \quad 1.411141478$

O $2.734213028-1.008675474 \quad 0.710916991$

C $3.109283364-2.122738543-0.090436381$

O $0.095225765-1.0485555972 .049673198$

C $0.709121941-2.1973543672 .666027880$

O

C $-4.462909377 \quad 0.474073058 \quad 0.117437974$

Bi -1.8054009872 .426988471 -2.883828530

C $-1.053959960 \quad 0.395609870-3.252034244$

C $-1.874155917-0.717095486-3.062645572$

C $0.2972543990 .286252689-3.598786463$

C -1.304764052 -1.982376889-3.231274166

C $0.846864222-0.989709761-3.752498280$

C $0.045782383-2.117716244-3.564895883$

C $-3.1289978413 .510958763-1.511385132$

C $-2.932707832 \quad 3.459721220-0.133438660$

C $-4.1406625994 .264114783-2.111497121$

C $-3.797655718 \quad 4.202163702 \quad 0.674822459$

C $-4.9951958814 .991071652-1.277712785$

C $-4.824150170 \quad 4.960090363 \quad 0.106807064$

C $-0.8607071273 .868341204-4.208234560$

C $-0.179615650 \quad 3.441955576-5.353105126$

C $-1.053061104 \quad 5.229451936-3.947892133$

C $0.333238708 \quad 4.396886044-6.233881948$

C $-0.538658327 \quad 6.173805313-4.839031581$

C $0.155996742 \quad 5.759041808$-5.977787187

C $-5.087731385-0.8969064860 .226733472$

C $-5.387743957-1.626805773-0.933785348$

C $\quad-5.305070868$-1.487575404 1.477417532
C $-5.891688207-2.923792327-0.838929525$

C $-5.814706178-2.7852086751 .574005293$

C $-6.104673221-3.5067136050 .414797964$

C $1.584567035-1.7591840063 .813392243$

C $2.960735806-2.0056983073 .820560651$

C $1.007810385-1.0842402164 .897185299$

C $3.747343651-1.6046550754 .903442812$

C $1.791470321-0.6722288215 .974381411$

C $3.164029257-0.9376966475 .982632241$

C $3.673545571-1.763165668$-1.452660210

C $4.101810832-0.466773510-1.755763930$

C $3.781333136-2.765005011-2.425343047$

C $4.623332220-0.177983725-3.019176168$

C $4.320594528-2.480876995-3.680164791$

C $4.740814294-1.181749989-3.982333190$

C $3.565437537 \quad 2.670175270 \quad 2.604708507$

C 3.2756360932 .0714895003 .835454432

C $3.385707445 \quad 4.0541662372 .456672269$

C 2.8161168912 .8439605034 .905749195

C $2.927999750 \quad 4.826237540 \quad 3.523104065$

C $2.640778607 \quad 4.2195079214 .751502055$

H $0.520120143 \quad 2.891577390 \quad 1.182901965$

H $\quad-0.723483175 \quad 0.161601129-0.903421935$

H 0.121530419 -1.862522718 0.130370130

H $1.628322990-0.187092586-0.843999918$

H $1.711406000 \quad 0.985657729 \quad 1.962113918$

H $\quad-0.040118567 \quad 4.998739180-0.901900148$

H $1.372049390 \quad 4.538013893 \quad 0.076567039$

H 1.8658881296 .157884952 -1.836161428

H $\quad 1.482804599 \quad 4.882212232-2.974309728$

H $3.752990267 \quad 4.807103081-0.886011728$

H 3.9441833374 .963846416 -2.638808422

H $3.343711658 \quad 3.447635289-1.936905870$

H $\quad-2.372552491 \quad-1.460158320 \quad 0.215948714$

H $\quad-2.461375529 \quad-0.269645440 \quad 1.529768439$ 
H $4.2227692590 .814766998 \quad 1.700577081$

H $4.792718925 \quad 2.2819361090 .863127296$

H $3.866268349-2.6496450550 .502585935$

H $2.266033582-2.820348666$-0.217044919

H $\quad-0.105119852 \quad-2.8378891843 .031506666$

H 1.288471755 -2.771410547 1.937695175

H $\quad-4.487342370 \quad 0.9884411991 .089975940$

H $\quad$-4.972751945 1.091168214 -0.623817969

H $\quad$-2.907778209 $-0.610054529-2.758576513$

H 0.9342588821 .156107211 -3.718707584

H $\quad$-1.924671819 -2.862268741 -3.084364374

H 1.900078813 -1.093712738 -3.996150144

H 0.482333789 -3.106416917 -3.672555923

H $\quad-2.175582538 \quad 2.8242343650 .309767087$

H $\quad$-4.297184756 4.277172205 -3.184555429

H $\quad-3.674231737 \quad 4.168253257 \quad 1.753608539$

H $\quad-5.7998200225 .568830579-1.722519756$

H $\quad \begin{array}{llll}-5.499207324 & 5.521351525 & 0.745944834\end{array}$

H $\quad-0.057769903 \quad 2.385870549$-5.576780324

H $\quad-1.604415728 \quad 5.561429921-3.071544674$

H $0.860803204 \quad 4.073131361$-7.126209612

H $\quad-0.689135310 \quad 7.231852364$-4.645391897

H $0.549315905 \quad 6.496201180-6.670958855$

H $\quad$-5.242153588 -1.164392257 -1.905512418

H $\quad-5.074106207 \quad-0.928885443 \quad 2.382702531$

H -6.128972433 -3.480102669 -1.742702040

H $\quad-5.983176545 \quad-3.2315718472 .550997871$

H $\quad$-6.500034682 4.5168918730 .486573490

H $3.418745206 \quad-2.5132427802 .976500108$

H $\quad-0.060140442-0.8818354034 .890062616$

H 4.814409066 -1.809176013 4.902765318

H $\quad 1.331776973$-0.155094174 6.812276047

H $3.774899899-0.625426276 \quad 6.825045111$

H $4.0040997890 .318613250-1.014348075$

H $3.447609216-3.775803699-2.198073480$
H $4.947833124 \quad 0.834344562 \quad-3.245942254$

H 4.411254477 -3.269486096 -4.421993880

H $5.157724251-0.957499239-4.960112400$

H 3.3979951870 .9989666053 .958839260

H $3.614339415 \quad 4.522149421 \quad 1.500934804$

H $2.590442148 \quad 2.362216553 \quad 5.853073757$

H 2.8001997595 .8993484653 .403436933

H $2.283893054 \quad 4.821099447 \quad 5.583364096$

O

S $\quad-4.730212340 \quad 1.616540997-4.252113035$

O

O $\quad-5.822923616 \quad 2.546145366$-4.496492269

C $-4.6714604650 .408032399-5.671463188$

F -4.595050922 1.062224424 -6.825324189

F $-3.595559343-0.377455747-5.530401792$

F $\quad-5.772513776-0.339247165-5.641340531$

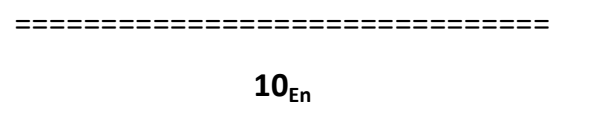

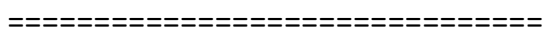

C $1.680381582 \quad 2.395549787 \quad 2.791653347$

O

C $\quad-0.712820058 \quad 1.168988397-0.804989675$

C $0.232919955 \quad 1.7811781770 .250756365$

C $-0.268724458 \quad 1.324656312 \quad 1.631295766$

C $0.852082560 \quad 1.192064847 \quad 2.758448490$

S $3.328004970 \quad 2.272443158 \quad 2.800036954$

C $3.928846475 \quad 4.027629787 \quad 2.796590264$

C $5.393803617 \quad 4.058653775 \quad 2.336368798$

C $5.603796495 \quad 3.6604067220 .870748391$

C $-0.306859498 \quad 1.538788223-2.235390154$

O $1.678740367 \quad 0.083595382 \quad 2.633993785$

C $1.133170668-1.2054424412 .995290071$

O

C $\quad-2.576949114 \quad 1.713990051 \quad 2.266219224$

O $1.581428049 \quad 1.373158391 \quad 0.064580226$ 
C $2.407651570 \quad 2.266626669-0.688237486$

O $-1.2309318810 .881207733-3.088814076$

C -0.9552000210 .989266187 -4.510443417

Bi -2.084050697 -1.352895463 -1.813547471

C $-0.508658815-1.937856295-3.234618502$

C $-0.795145120-2.621600646-4.413001451$

C $0.798002760-1.587798523-2.882529904$

C $0.263440209-2.949594361-5.268028320$

C $1.842888548-1.922699418-3.747185212$

C $1.574016393-2.599065666$-4.940819386

C $-2.230259354-3.024114023-0.406075993$

C -1.202700698 -3.177622893 0.529419434

C $-3.247131103-3.982535432-0.509181575$

C -1.183292018 -4.295884672 1.365626549

C $-3.234823944-5.0853414370 .350379510$

C $-2.204044059-5.247117174 \quad 1.281645848$

C $-3.794410881-0.042644578-1.341160673$

C $-3.980380322 \quad 1.236124924-1.866845604$

C $-4.712090294-0.600968614-0.447917125$

C $-5.087505864 \quad 1.979975722-1.450341641$

C $-5.8128817170 .155880341-0.039217885$

C $-5.997063245 \quad 1.446671195$-0.535563047

O $-3.622434278-2.474424520-3.170236645$

S $-4.563295428-1.625364923-4.051607108$

O

O $-3.836473121-0.457952353-4.593262590$

C $-4.806164003-2.770300615-5.499506861$

F $-5.600187853-2.175455221-6.390159714$

F $-3.622404289-3.035398341-6.082310436$

F $\quad-5.359475143-3.916148642$-5.099863202

C -1.3358550112 .355686028 -5.017334605

C $-0.372084695 \quad 3.348389436-5.235736159$

C $-2.6939166272 .656683827-5.217186465$

C $-0.7545825054 .630446961-5.645154349$

C $-3.075429540 \quad 3.937751240-5.619655365$
C $-2.107290988 \quad 4.925967709-5.834653128$

C $3.420793091 \quad 1.457722336-1.464875358$

C $4.017761193 \quad 0.327846677 \quad 0.879435471$

C $3.763277124 \quad 1.816778510-2.775915188$

C $4.941745160-0.433718381-1.602439058$

C 4.6965698131 .060416666 -3.495847330

C $5.285763487-0.067080133-2.910789689$

C $-2.582380392 \quad 0.502675152 \quad 3.164077491$

C $\quad-2.231785728 \quad 0.624316979 \quad 4.517932350$

C $-2.850253802-0.766710102 \quad 2.641658263$

C -2.150641654 -0.5069018195 .329951082$

C $-2.779778078-1.9011313593 .454301027$

C $-2.425745554-1.7713650074 .798103533$

C $1.842751588-2.2411390322 .163251146$

C $2.325487493-3.4231198862 .730935048$

C $1.990268501-2.0247747480 .784895318$

C $2.932659695 \quad-4.391669148 \quad 1.926483927$

C $2.599764183-2.989834251-0.014732656$

C $3.067883141-4.1773376200 .554132780$

H $1.179964455 \quad 3.3632050892 .765162006$

H $\quad-1.700881634 \quad 1.622990701$-0.618115072

$\begin{array}{lllll}\text { H } & 0.152636827 & 2.879996324 & 0.216641013\end{array}$

H $\quad-0.663170492 \quad 0.309696008 \quad 1.541950115$

H $0.242749406 \quad 1.178302717 \quad 3.684813493$

H $3.282740005 \quad 4.599897734 \quad 2.124170729$

H 3.8174508374 .4041489893 .817366700

H $5.999693133 \quad 3.426797283 \quad 2.998234413$

H $5.743177055 \quad 5.085324238 \quad 2.498579248$

H $\quad \begin{array}{llll}5.307323511 & 2.624858601 & 0.667351631\end{array}$

H $\quad 6.659121407 \quad 3.752153690 \quad 0.599315520$

H $5.031360438 \quad 4.3098926650 .198379291$

H $\quad-0.353000244 \quad 2.627866907$-2.387299962

H $0.710236081 \quad 1.185581223-2.454210053$

H $\quad 1.277654372-1.3725602844 .070348517$

H $\quad 0.052529524 \quad-1.218241518 \quad 2.801343128$ 
H $\quad-3.126279386 \quad 2.549603708 \quad 2.708038024$

H $\quad \begin{array}{llll}3.025091098 & 1.478653406 & 1.293017347\end{array}$

H $1.810821032 \quad 2.883875320-1.374658715$

H $2.910233656 \quad 2.959939270 \quad 0.009182446$

H $0.105461553 \quad 0.756358776$-4.683000738

H $\quad-1.5718904990 .212370198$-4.963266126

H -1.810022298 -2.889679936 -4.674403810

H $1.001007613-1.052395274-1.962058136$

H $0.051382699-3.474142854$-6.196101452

H 2.859762934 -1.637816273 -3.487522055

H 2.385829164 -2.850162455 -5.618171343

H $\quad-0.412978710-2.4398699760 .600799077$

H $-4.028437251-3.881424744-1.256883888$

H $\quad-0.365602923 \quad-4.4178222512 .071230219$

H $\quad-4.025987016-5.8273649330 .278074742$

H $\quad-2.191992884-6.119104140 \quad 1.929538330$

H $\quad-3.293882577 \quad 1.640882889-2.598332982$

H $\quad$-4.596670027 -1.615817771 -0.077936390

H $\quad-5.240975823 \quad 2.975668106-1.858384586$

H $\quad-6.530440953-0.2743594250 .653672021$

H $\quad-6.857958658 \quad 2.030690495 \quad-0.222112421$

H $0.683113466 \quad 3.112174418$-5.093485580

H $\quad-3.438343894 \quad 1.877171551$-5.065092773

H $0.000453430 \quad 5.394260162$-5.820444925

H $\quad-4.1277931794 .164630667-5.779130733$

H $\quad-2.4065149325 .921967456-6.155210465$

H $3.728184418 \quad 0.0258351790 .124605572$

H $3.294977810 \quad 2.686984491 \quad$-3.239641144

H $5.383976790-1.319415485$-1.147408088

H $4.951965621 \quad 1.345034137$-4.515569637

H $6.004584852-0.660488332-3.474740317$

H $\quad-2.033230187 \quad 1.610595194 \quad 4.933272944$

H $\quad-3.105050942-0.8670176461 .592652886$

H $-1.884628501 \quad-0.4046694536 .378616091$

H $\quad-2.983129761 \quad-2.878923912 \quad 3.028783908$
H $-2.366891723 \quad-2.651453185 \quad 5.432512410$

H $2.226381053 \quad-3.5916521793 .801124062$

H $1.615009969-1.1037866740 .348690825$

H $3.301427681 \quad-5.3108179822 .372894896$

H 2.693552029 -2.821246900 -1.083512917

H $3.534994101-4.933327110-0.071150654$

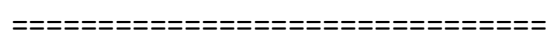

$10-\beta_{\text {ch }}$

C $\quad-0.547219700 \quad 0.335353991-1.882457921$

O

C $\begin{array}{llll}-1.352679369 & 0.167360392 & 0.392615384\end{array}$

C $-0.197667515-0.777462875 \quad 0.749892634$

C $0.125994988-1.683425151-0.453214225$

C $0.426980714-0.838362887-1.706766824$

S $0.1036285371 .671860208-2.967785346$

C $1.784809137 \quad 1.952568561-2.225800903$

C $2.312917777 \quad 3.368059737-2.463999035$

C $2.437285078 \quad 3.758707689-3.939674286$

C $-1.694148722 \quad 1.074966842 \quad 1.569946769$

O $0.310218388-1.580679071-2.903473449$

C $1.469230093-2.353689925$-3.287736148

O $1.325662037-2.405443736-0.232080171$

C $1.220761708-3.6187348430 .512268685$

O $0.922007142 \quad 0.0663585780 .988893322$

C $1.802781569-0.2846257152 .080242166$

$\begin{array}{llll}\text { O } & -2.473723683 & 2.175971910 & 1.130766925\end{array}$

C $-2.668188366 \quad 3.168824911 \quad 2.174221403$

Bi $-1.904766963 \quad 3.482112203-1.650798018$

C -0.4875102014 .264696887 -0.168848539

C $-0.603822432 \quad 5.643644404 \quad 0.022168555$

C $0.458656613 \quad 3.4934511850 .502662231$

C $0.277553411 \quad 6.264576397 \quad 0.912481306$

C 1.3382634954 .1361280421 .378256110

C $1.247451787 \quad 5.515379188 \quad 1.580931392$ 
C $-1.8323700314 .929802466-3.289697584$

C $-2.991605347 \quad 5.173110777-4.037828921$

C $-0.657088907 \quad 5.650863419-3.530154917$

C $-2.9595566496 .142851919-5.043643449$

C $-0.639633817 \quad 6.616279016-4.539721008$

C $-1.788161058 \quad 6.859481992-5.298053353$

C $-3.284127951 \quad 1.904525589$-2.290516641

C -3.3334841331 .680456558 -3.666888717

C $-4.084521453 \quad 1.215386333-1.382603120$

C $-4.219470674 \quad 0.711603722-4.147422034$

C $-4.9647933830 .254684976-1.887687760$

C $-5.0279141830 .000535961-3.259522786$

O $-3.4406423654 .587153641-0.589386019$

S $-4.9993261594 .471193512-0.696141629$

O

$\begin{array}{llll}\text { O } & -5.521468833 & 3.458387826 & 0.221491149\end{array}$

C $\quad \begin{array}{llll}-5.390869904 & 6.126748083 & 0.063787757\end{array}$

F $-6.711914237 \quad 6.280207273 \quad 0.105475729$

F $-4.892195076 \quad 6.167680284 \quad 1.303283281$

F $-4.8440762617 .098453752-0.669436201$

C $-3.393658814 \quad 2.607422695 \quad 3.369817922$

C $-4.742588912 \quad 2.236226633 \quad 3.251158506$

C $-2.729613228 \quad 2.405546357 \quad 4.585892663$

C -5.4116400691 .6721206014 .337528805$

C $-3.401660908 \quad 1.845836813 \quad 5.676282617$

C $-4.743227180 \quad 1.4764107715 .551529796$

C 3.0526174950 .5461941651 .893247591

C 3.6760643230 .5540843710 .629770167

C $3.582024164 \quad 1.339906770 \quad 2.922552774$

C $4.803406137 \quad 1.3498659220 .397924539$

C $4.720207576 \quad 2.131917902 \quad 2.693785874$

C $5.329169257 \quad 2.142461899 \quad 1.431336847$

C $2.617677483-4.175579930 \quad 0.700943589$

C $3.720969473-3.3138002390 .829521175$

C $2.823819254-5.559425996 \quad 0.772951279$
C $5.006407447-3.830028501 \quad 1.029529249$

C $4.109417660-6.0755237020 .979972148$

C $5.203942186-5.2123742351 .108107916$

C $2.673193924-1.471467223-3.532358523$

C $3.726882796-1.416446248-2.603295870$

C $2.714229331-0.642588386-4.666400868$

C $4.805834760-0.545143389-2.803330010$

C $3.7925208330 .222639730-4.872214025$

C $4.838865250 \quad 0.276881045$-3.935974099

H $-1.447517200-0.035015165-2.388953305$

H $\quad-2.236747026 \quad-0.3920438270 .052109994$

H $-0.454094742 \quad-1.377124826 \quad 1.634811882$

H $\quad-0.720810632$-2.356816029 0.666026719

H $1.443289498-0.463610183-1.560139660$

H $1.7107478691 .734515562-1.159869519$

H $2.441723201 \quad 1.217766809$-2.695227663

H $3.300117516 \quad 3.401683043-1.984575297$

H $\quad 1.693910347 \quad 4.089189025$-1.918949925

H $3.045808953 \quad 3.030275316-4.485043381$

H $2.908871451 \quad 4.741104314$-4.042129164

H $1.459042021 \quad 3.813423626$-4.431124713

H $\quad-2.2328738990 .483008335 \quad 2.322980010$

H $\quad-0.760440330 \quad 1.430708671 \quad 2.025912870$

H 1.689258535 -3.110444093 -2.527173121

H $1.153138743-2.855121381$-4.209097911

H $0.750478343 \quad-3.423241636 \quad 1.492949914$

H 0.579002198 -4.340956468 0.021442710

H $1.314550521-0.072337303 \quad 3.057475598$

H 2.047459481 -1.360017277 2.042404642

H $-1.684451861 \quad 3.571425376 \quad 2.458589071$

H $\quad-3.242313427 \quad 3.958149949 \quad 1.691794081$

H $\quad-1.363681783 \quad 6.229706550-0.486124079$

H $0.517300238 \quad 2.422683690 \quad 0.367668869$

H $\quad 0.197463677 \quad 7.334895107 \quad 1.079365588$

H $2.088952282 \quad 3.545532958 \quad 1.897459553$ 
H $\quad 1.931448983 \quad 6.008335350 \quad 2.265676631$

H $\quad-3.9183390864 .647978079$-3.828207831

H $\quad 0.236077574 \quad 5.485862378$-2.933784704

H $\quad-3.858526594 \quad 6.340577700 \quad-5.621012028$

H $\quad 0.269324074 \quad 7.180818932$-4.727919886

H $\quad-1.772551477 \quad 7.613306845$-6.079872029

H $\quad-2.711973203 \quad 2.238786097$-4.361493957

H $\quad-4.046737227 \quad 1.433898764$-0.322332013

H $\quad-4.2761453410 .521912068$-5.215185541

H $\quad$-5.609917273 -0.285049776 -1.200512651

H $\quad$-5.716859712 -0.748111443 -3.639521785

H $\quad-5.258474095 \quad 2.404742693 \quad 2.309320704$

H $\quad-1.682478540 \quad 2.691949338 \quad 4.680414610$

H $\quad-6.458577811 \quad 1.3918694894 .242284491$

H $\quad \begin{array}{llll}2.880108826 & 1.698819992 & 6.619386812\end{array}$

H $\quad \begin{array}{llll}-5.268698475 & 1.040864773 & 6.398156644\end{array}$

H 3.262360648 -0.053520453 -0.175795676

H $3.101649114 \quad 1.3482424463 .903583006$

H $5.266969987 \quad 1.352303347 \quad-0.590567877$

H $\quad 5.125642045 \quad 2.744808670 \quad 3.501003368$

H $\quad 6.207661284 \quad 2.764936375 \quad 1.252851552$

H $3.579218692-2.2392229950 .757649170$

H 1.978406809 -6.239460148 0.661190460

H 5.852241297 -3.149216305 1.122915849

H 4.257647683 -7.152738349 1.030322939

H 6.204995635 -5.614541718 1.262115601

H 3.698066168 -2.056485756 -1.722208711

H $1.893584301-0.675744621-5.385339825$

H $5.620887163-0.516393434-2.078566709$

H $3.822112703 \quad 0.849497464$-5.763015095

H $5.679377113 \quad 0.949997606$-4.097881634

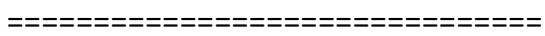

S5

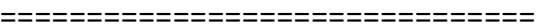

C $\quad$\begin{tabular}{llll}
\hline .763378986 & 0.166109752 & -0.830174509
\end{tabular}
O $1.131198497-0.996656812-1.306015244$

C $0.992090164-2.051597971-0.300454401$

C $0.259388868-1.5201712170 .938835631$

C $0.952922904-0.249361681 \quad 1.488500239$

C $1.044354521 \quad 0.7879113150 .364515986$

S $1.725232323 \quad 1.383942083$-2.240061489

C $3.529958471 \quad 1.695813257-2.532869848$

C $3.752933542 \quad 2.787653954-3.581760776$

C $5.248025783 \quad 3.119734052$-3.683632131

C $0.227181689-3.173279939-0.978783044$

O $1.784551046 \quad 1.929757536 \quad 0.733852743$

C $0.976679254 \quad 3.103311656 \quad 1.035816707$

$\begin{array}{llll}\text { O } & 0.196197949 & 0.366499005 & 2.507462537\end{array}$

C $0.358526953-0.1629191823 .836453080$

O

C $-2.114946704-1.513702367 \quad 1.499824371$

O 1.013325768 -3.674320088

C $0.347728792-4.689509287-2.816620397$

Bi 0.882676525 -0.219504796 -4.165811437

C $-1.266303164-0.060795660-3.724594529$

C $-2.0358559820 .299489270-4.834898180$

C $-1.778542777-0.155170117-2.434304211$

C $-3.3878994870 .582172938-4.628043022$

C $-3.1336639370 .144139836-2.256595557$

C $-3.9299707850 .509359085-3.343083882$

C $1.1442256391 .317986834-5.698818677$

C $2.158305768 \quad 1.246132056$-6.665960533

C $0.350722510 \quad 2.470286787 \quad-5.579018265$

C $2.346395758 \quad 2.317751411$-7.540600929

C $0.560257302 \quad 3.538553331-6.452038267$

C 1.5495362153 .460268193 -7.434995405

C $2.506502117-1.666291981-4.477740598$

C $3.272399395-2.142746276-3.416539118$

C $2.740481990-2.034424362-5.807103934$

C 4.337553935 -2.999989902 -3.710142557 
C $3.806513840-2.896998911-6.073781719$

C $4.604239552-3.372058594-5.028934898$

C $-0.616550808-4.097225855-3.821124436$

C $-0.156846503-3.744885681-5.099827926$

C $-1.959297128-3.857297741-3.502617860$

C $-1.014524802-3.158253750-6.035191889$

C $-2.819794460-3.266279885-4.432554470$

C $-2.350757898-2.916734664-5.699076971$

C $-3.225149839-0.5148506441 .312196072$

C $-4.513238386-0.9118169110 .944339336$

C $-2.950215257 \quad 0.850047791 \quad 1.490708723$

C $\quad \begin{array}{llll}-5.516465141 & 0.043336686 & 0.745177297\end{array}$

C $-3.945492979 \quad 1.802160212 \quad 1.281704700$

C $\quad \begin{array}{llll}-5.232180938 & 1.399890483 & 0.905236714\end{array}$

C $1.760171377-0.0168738274 .380690793$

C $2.282295082-0.9924626815 .236841371$

C 2.5308973561 .1113923194 .071199140

C $3.552522264-0.835717867 \quad 5.793258195$

C 3.8058982591 .2603355574 .615407431

C $4.316206243 \quad 0.291078917 \quad 5.483253426$

C $0.416893355 \quad 3.701485295 \quad-0.231453206$

C $-0.8523327613 .340628689-0.704235366$

C $1.2240599634 .533748436-1.021863559$

C $-1.294403844 \quad 3.775613124-1.956970824$

C $0.779157073 \quad 4.984200202-2.264440513$

C $-0.4795964594 .597779945-2.737582395$

H $2.824998379-0.003330028$-0.596598316

H $2.001862054-2.390047873-0.028863546$

H $0.278238932-2.3127290581 .699590679$

H $1.966134088-0.4932255851 .841482113$

H $0.020972577 \quad 1.031843708 \quad 0.062108913$

H $3.877447323 \quad 2.015564437$-1.544460006

H $4.012541050 \quad 0.748939018$-2.795438807

H 3.1787904563 .677687606 -3.302541613

H $3.377923335 \quad 2.456933887$-4.554792633
H $5.643176339 \quad 3.488275353-2.731411327$

H $5.410506107 \quad 3.896647955$-4.435542467

H $5.834471924 \quad 2.242693334$-3.978302030

H $0.017115041-3.956829899-0.231831095$

H $\quad-0.733372906$-2.776160616 -1.327816799

H $0.193588295 \quad 2.821734829 \quad 1.745420869$

H $1.669544166 \quad 3.787414387 \quad 1.530992517$

H $\quad-0.361209557 \quad 0.410484510 \quad 4.429641709$

H $\quad 0.044013127 \quad-1.216374702 \quad 3.873949879$

H $\quad-2.465385007$-2.540566493 1.330436804

H $-1.715102704-1.4507770632 .516616628$

H $\quad 1.151270014-5.227577131$-3.326567989

H $\quad-0.163971665$-5.395766479 -2.147447036

H $\quad-1.6173250530 .374448366-5.833311395$

H $\quad-1.182600500 \quad-0.446069725$-1.578609748

H $\quad-4.0068722310 .863536673 \quad-5.474975354$

H $\quad-3.554365550 \quad 0.089292634$-1.259749322

H $\quad-4.979099477 \quad 0.741321354-3.185364740$

H $2.812457900 \quad 0.384882542 \quad-6.744163953$

H $\quad-0.415642020 \quad 2.553516009$-4.814182680

H $3.121494740 \quad 2.260345969$-8.299091410

H $\quad-0.049715005 \quad 4.432699682$-6.362383487

H $\quad 1.705339244 \quad 4.292311732$-8.115134174

H 3.033119241 -1.895272569 -2.391313168

H $2.109498232-1.694518352$-6.623116646

H 4.947316045 -3.387019878 -2.898796205

H $4.003015061 \quad-3.199348718$-7.098152517

H 5.430056525 -4.043305825 -5.244256656

H $\quad 0.870822262 \quad-3.968156536$-5.371353958

H $\quad-2.344802013$-4.155712618 -2.530651882

H $\quad-0.655023351 \quad-2.932643358$-7.036709923

H $\quad$-3.859471126 -3.091499876 -4.171691480

H $\quad-3.026183165$-2.477877820 6.427793559

H $\quad-4.736888889-1.9671532750 .808267048$

H $\quad-1.953244290 \quad 1.144716968 \quad 1.803568858$ 
H $\quad-6.516999372 \quad-0.2738894970 .466106128$

H $\quad-3.726758692 \quad 2.856979453 \quad 1.425713893$

H $\quad-6.010636298 \quad 2.1413096090 .750335853$

H 1.691935593 -1.874248779 5.478384832

H $2.133982356 \quad 1.858314753 \quad 3.390646197$

H 3.946325651 -1.594240848 6.463864622

H $4.399241986 \quad 2.137427802 \quad 4.370736116$

H $5.305723280 \quad 0.412338212 \quad 5.913944376$

H $\quad-1.506066943 \quad 2.721399883-0.095180491$

H $2.203654683 \quad 4.832505038$-0.655474417

H $\quad-2.2795468123 .484411418$-2.311102260

H 1.4015298365 .650529945 -2.855674005

H $\quad-0.833712868 \quad 4.963600834-3.697687656$

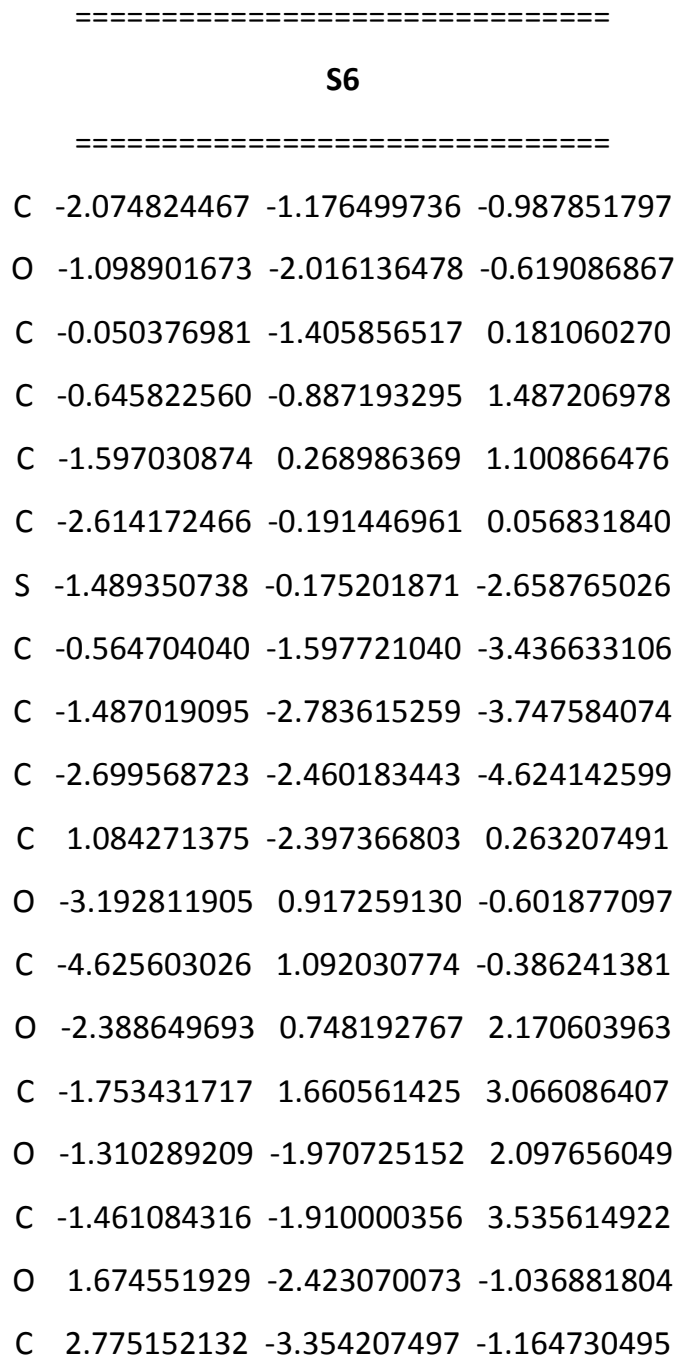

Bi $0.434604608 \quad 1.604253887$-2.412449186

C $-0.733586993 \quad 3.367613796-2.935482960$

C $-2.1085080793 .360093445-2.680800636$

C $-0.0856747354 .453956071-3.536467069$

C $-2.8532524514 .481324250-3.054056533$

C $-0.853572074 \quad 5.563695061-3.897686630$

C $-2.230057212 \quad 5.574171182-3.661108810$

C $1.518271437 \quad 1.715820705-0.515020319$

C $\quad 1.211587624 \quad 2.772020400 \quad 0.350844773$

C $2.5493683980 .800111039-0.255456558$

C $1.984691298 \quad 2.913626557 \quad 1.507352459$

C 3.2959917540 .9576407210 .911546163

C $3.012808373 \quad 2.011299907 \quad 1.787370935$

C $1.7535138070 .898106332-3.986673779$

C 1.8202607851 .619672067 -5.184672681

C $2.527975533-0.250989597-3.767030467$

C $2.709581167 \quad 1.191729062-6.173491169$

C $3.405532136-0.661303967-4.770485661$

C $3.5006354540 .060367032-5.964381755$

C $4.013717622-2.847875097$-0.468594614

C $4.846033358-1.916664271-1.107433444$

C 4.314992015 -3.238481164 0.842509570

C $5.952719392-1.379627119-0.448966691$

C $5.421085634-2.7023554881 .506653623$

C $6.239835229-1.770909150 \quad 0.863066065$

C $-0.140808630-1.6968250274 .240533864$

C $0.014846044-0.691458040 \quad 5.201370982$

C $0.966542403-2.4885749413 .898475708$

C $1.255328015-0.4749697305 .809705536$

C $2.208277335-2.2647543974 .491925404$

C $2.355599359-1.2540783245 .448892444$

C $-1.584089823 \quad 3.0639136372 .515924355$

C $-0.808420720 \quad 3.978950843 \quad 3.240090782$

C $-2.1947310393 .479647914 \quad 1.329463499$

C $-0.638774497 \quad 5.285208193 \quad 2.782119796$ 
C $-2.011882667 \quad 4.784487088 \quad 0.860370266$

C $-1.233481598 \quad 5.690001521 \quad 1.582504004$

C $-5.405252364-0.091394671-0.889714320$

C $-5.419839519-0.382659422-2.263912002$

C $-6.058758066-0.951395950 \quad 0.000549575$

C $-6.081026702-1.515800576-2.736716126$

C $-6.723610064-2.086669280-0.472655996$

C $-6.732433096-2.370773249-1.838900638$

H $\quad$-2.887858469 -1.720827611 -1.475998271

H $0.330537732-0.548469202-0.379173087$

H $0.169780018-0.4990596852 .112281934$

H $\quad-0.988303377 \quad 1.0816329790 .678695852$

H $\quad-3.366243548 \quad-0.7766112480 .602368173$

H $\quad$-0.137180630 -1.178353769 -4.350581369

H $0.226988423-1.896690213-2.744731828$

H $-1.789532212-3.266545122-2.812772905$

H $\quad-0.839640789$-3.508884198 -4.257673440

H $\quad-3.422399024-1.821308533$-4.104195290

H -3.221114930 -3.380056452 -4.901883821

H $\quad-2.400661368$-1.952373962 -5.547566264

H $1.801834179-2.0637457381 .024325556$

H 0.705866179 -3.387964839 0.547145663

H $\quad-4.798229014 \quad 1.272796350 \quad 0.680562705$

H $\quad-4.8601018332 .005491035$-0.940714434

H $\quad-2.402559921 \quad 1.680422774 \quad 3.948606028$

H $\quad-0.780999483 \quad 1.267332564 \quad 3.396132752$

H $\quad-2.182659268$-1.131744575 3.805217133

H $\quad-1.894290257 \quad-2.882501236 \quad 3.788439402$

H 2.469140039 -4.336549474 0.780300131

H 2.936739768 -3.445094760 -2.243244752

H $\quad-2.5958106602 .522698388$-2.190830836

H 0.9848061514 .458760830 -3.724254384

H $\quad$-3.923305735 4.496867572 -2.868549924

H $\quad-0.3717297926 .416243899-4.366719995$

H $\quad-2.8192624296 .438732179$-3.951031424
H $\quad 0.399956055 \quad 3.469093987 \quad 0.163241043$

H $2.774535895-0.034715942-0.908371158$

H $1.758714901 \quad 3.728629426 \quad 2.186141716$

H $4.0969935190 .253759004 \quad 1.120896130$

H $3.597508010 \quad 2.128565115 \quad 2.694880284$

H $1.201535792 \quad 2.495993698$-5.359442258

H $2.436190326-0.839707242$-2.859257468

H $2.778968849 \quad 1.743730198$-7.105885399

H 4.012597148 -1.549631181 -4.621173714

H $4.188298681-0.266017185$-6.738549070

H 4.637403005 -1.622355646 -2.134306807

H 3.689345095 -3.975532671 1.341732075

H $6.599250032 \quad-0.671012856-0.959775314$

H 5.652154947 -3.020388725 2.519538892

H 7.108232218 -1.363875068 1.373475913

H $-0.840418086-0.0818679915 .485891632$

H $0.844399147 \quad-3.280341727 \quad 3.162725283$

H $1.360692201 \quad 0.300993507 \quad 6.563754680$

H $3.059859402-2.8837865814 .219752453$

H $3.320382899-1.0848591095 .919790167$

H $\quad-0.339742954 \quad 3.6693621294 .172317708$

H $\quad-2.802326480 \quad 2.779734826 \quad 0.769549529$

H $\quad-0.048013846 \quad 5.989817421 \quad 3.360739269$

H $\quad-2.486217079 \quad 5.093673515$-0.067256041

H $\quad-1.101929481 \quad 6.707239724 \quad 1.225620503$

H $\quad-4.922788194 \quad 0.290399347$-2.960605664

H $\quad-6.056380359 \quad-0.7281141881 .065669052$

H $\quad-6.104961980$-1.726994685

H $\quad-7.234443241 \quad-2.744835508 \quad 0.224704221$

H $\quad-7.253572924 \quad-3.249744278$-2.207662681

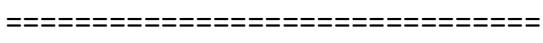

11

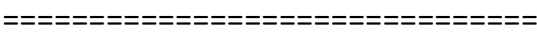

C $1.378876534-0.259626921-2.450703067$

O $1.748488460 \quad 0.740189804-1.773242651$ 
C $\quad 1.441656260 \quad 0.847648248-0.290964634$

C $1.282785489-0.5450383550 .291388086$

C $0.223786035-1.334777801-0.495128604$

C $0.744275122-1.519089844-1.925173230$

C $2.591280892 \quad 1.661942947 \quad 0.311144210$

O $-0.117135583-2.062175586-2.888113332$

C $-1.369387214-1.351163784-3.076114124$

O $\quad-0.003980280-2.609739713 \quad 0.058525442$

C $-1.107920585-2.6579301590 .989848449$

O $2.561097356-1.1573545650 .230005939$

C $2.835539645-2.198353043 \quad 1.210533347$

O $2.325552779 \quad 3.045676286 \quad 0.315052112$

C $1.794449316 \quad 3.5309207541 .558968192$

C $0.426041074 \quad 2.963793272 \quad 1.871421997$

C $-0.697708299 \quad 3.432067252 \quad 1.175438816$

C $0.267781644 \quad 1.917642675 \quad 2.788158679$

C $-1.9555486192 .873410276 \quad 1.398170949$

C $-0.990947647 \quad 1.352101994 \quad 3.012285826$

C $-2.104664215 \quad 1.833853892 \quad 2.322236373$

C $2.615236052-1.7146313532 .621258409$

C $1.545295813-2.1957244313 .385167703$

C $3.434884831-0.7091867573 .155188807$

C $1.286448240-1.6752798164 .655810129$

C $3.181085312-0.1877594504 .423642511$

C $2.100261711-0.665733398 \quad 5.173343472$

C $-2.428428300-2.477805870 \quad 0.286969931$

C $-2.903721447-3.484888391-0.563178261$

C $-3.139470578-1.2760163890 .388387570$

C -4.069839485 -3.291359531 -1.300959301

C $-4.306190403-1.077039092-0.355752657$

C $-4.768302892-2.083628246-1.204615809$

C $-1.1531120320 .129424275 \quad-3.269924972$

C $-1.5738564941 .059938890-2.303521513$

C $-0.5010139700 .600994103-4.427498709$

C $-1.2998050702 .415850853-2.464105516$
C $-0.235798646 \quad 1.959532329-4.590871747$

C $-0.6221885492 .865837250-3.601523798$

H $1.691165274-0.234248676$-3.492730846

H $0.510408960 \quad 1.413708545 \quad-0.270696322$

H $0.938055947 \quad-0.4108385391 .324581384$

H $\quad-0.699163105-0.746118897-0.514490382$

H $1.597468499-2.216753910-1.858863181$

H $2.785338932 \quad 1.270840411 \quad 1.317609429$

H $3.493798261 \quad 1.486483318$-0.281909570

H $\quad-2.041464026-1.544159653-2.238397646$

H -1.795317068 -1.812289466 -3.970013559

H $-1.028471843 \quad-3.6471622601 .448032380$

H $-0.975520693 \quad-1.9044127201 .778296308$

H $3.885701285-2.4452019821 .033392240$

H 2.220789710 -3.076915501 0.995308812

H 2.4961302793 .3075626042 .375556993

H $1.748926636 \quad 4.615977672 \quad 1.435944982$

H $\quad-0.580030313 \quad 4.245890590 \quad 0.463875170$

H $1.127321530 \quad 1.5430460063 .338371943$

H $\quad-2.823247940 \quad 3.2598852580 .870415365$

H $-1.0969857310 .548962093 \quad 3.735809725$

H $\quad-3.089086704 \quad 1.4175935342 .516909404$

H 0.918242535 -2.988245667 2.985052774

H $4.276965024-0.3411151082 .572875002$

H 0.456099703 -2.061730798 5.241415726

H 3.8282844650 .5830509854 .833882711

H $1.902461037-0.2603628916 .161595753$

H -2.345160425 -4.412142253 0.657559088

H $-2.771575433-0.4906839321 .043800375$

H -4.432977198 -4.077632554 -1.956125802

H $\quad-4.850460270 \quad-0.140440682-0.273298722$

H $\quad-5.673601777$-1.932409228 -1.785073566

H $\quad-2.125487551 \quad 0.715462561$ - 1.432717059

H $\quad-0.202977896-0.111463471 \quad-5.193109360$

H $-1.615072232 \quad 3.121996952 \quad-1.702854051$ 
H $0.267181450 \quad 2.311306361-5.486325635$

H $\quad-0.408323138 \quad 3.923251930-3.722620566$

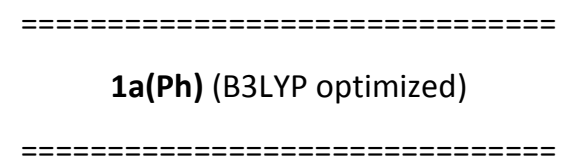

Bi $\quad-3.355037195 \quad 5.030922946 \quad 8.717709784$

C $-3.865183744 \quad 2.946675066 \quad 9.103619130$

C $-2.947722995 \quad 1.948222606 \quad 8.757899416$

C $-5.111315473 \quad 2.639286873 \quad 9.666352461$

C -3.2935634690 .6146832998 .986118641$

C $\begin{array}{llll}-5.438615320 & 1.298891185 & 9.883233449\end{array}$

C $\quad \begin{array}{llll}4.532183424 & 0.292579066 & 9.543994815\end{array}$

H $\quad-1.982009043 \quad 2.194906254 \quad 8.329089881$

H $\quad \begin{array}{llll}-5.825030038 & 3.412761445 & 9.938909854\end{array}$

H $\quad-2.590047130-0.170192686 \quad 8.726011165$

H $\quad-6.400628501 \quad 1.04602329010 .318162534$

H $\quad-4.792375007 \quad-0.7471997219 .716204117$

C $-4.219822792 \quad 5.711462607 \quad 6.827692166$

C $\quad-5.133495800 \quad 4.828970184 \quad 6.240311658$

C $-3.900834220 \quad 6.949890982 \quad 6.263577244$

C $\quad-5.751867821 \quad 5.2129713195 .048700654$

C $-4.5317760097 .308591538 \quad 5.071347327$

C $-5.451572802 \quad 6.446873836 \quad 4.469743237$

H $\quad \begin{array}{llll}-5.363140428 & 3.862517379 & 6.679953848\end{array}$

H $\quad-3.165742874 \quad 7.606542014 \quad 6.713180821$

H $\quad-6.460720202 \quad 4.5414584264 .574424967$

H $\quad-4.293782642 \quad 8.262870543 \quad 4.611667160$

H $\quad-5.931809734 \quad 6.736529575 \quad 3.540222076$

C $\quad-3.526949998 \quad 6.215370030 \quad 10.541954534$

C $-3.836236075 \quad 5.513053906 \quad 11.712489841$

C -3.3909761857 .60677075310 .517605153$

C $\quad-4.022577434 \quad 6.237472657 \quad 12.891783290$

C $-3.5828578718 .308393491 \quad 11.708348893$

C $-3.898801413 \quad 7.62775056612 .887025406$

H $\quad-3.928539083 \quad 4.431060399 \quad 11.723546237$
H $\quad-3.120188401 \quad 8.1363545219 .611548302$

H $\quad-4.260582914 \quad 5.710514763 \quad 13.810555039$

H $\quad-3.4792404839 .388998495 \quad 11.712245697$

H $\quad-4.044886403 \quad 8.184345492 \quad 13.807578468$

O $\quad-1.316826695 \quad 4.615225010 \quad 8.354120889$

S -0.4589200495 .8876090637 .900217638$

O $0.212668467 \quad 5.660276683 \quad 6.638532749$

O

C $0.819879230 \quad 5.927923697 \quad 9.269347221$

F $0.185622994 \quad 6.024748170 \quad 10.436568531$

F 1.5377165754 .8161988899 .227551082

F $1.589260398 \quad 6.9889153369 .077834867$

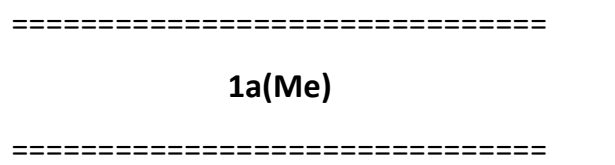

Bi $\quad-3.308439548 \quad 5.053104098 \quad 8.592370480$

O

S $\quad-0.350796568 \quad 5.8531665897 .981002332$

O $0.460941910 \quad 5.532527983 \quad 6.829334105$

O

C 0.7380902185 .9199141059 .508556316

F $\quad-0.061651558 \quad 6.033601516 \quad 10.576060702$

F 1.4474738564 .8092059799 .585579412

F $1.519421327 \quad 6.9795759449 .409211350$

C $-3.913637224 \quad 2.976759136 \quad 8.988064134$

H $\quad-3.9795042432 .4538169618 .033008558$

H $\quad-3.136813764 \quad 2.536547944 \quad 9.615561694$

H $\quad-4.877196533 \quad 2.9824273939 .501085471$

C $-3.947384461 \quad 5.845563114 \quad 6.641314473$

H $\quad-3.623516030 \quad 6.8853299786 .600600146$

H $\quad-3.444021184 \quad 5.248727680 \quad 5.878170475$

H $\quad-5.032051016 \quad 5.7429911396 .570815695$

C $-3.449800976 \quad 6.39407798910 .333166712$

H $\quad-4.479994625 \quad 6.368785855 \quad 10.693473426$

H $\quad-2.755154925 \quad 6.015305700 \quad 11.083926396$ 
H $\quad-3.155521256 \quad 7.385849724 \quad 9.991410123$

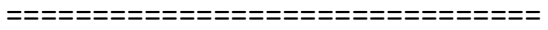

4- $\alpha(\mathrm{MeO})$

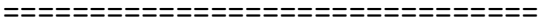

C $0.048704307 \quad-0.258646468 \quad 0.039797695$

O

C $-0.934463149-1.3328433901 .940664204$

C $0.147301311 \quad-0.7987726712 .895072863$

C $1.458118772-0.5871690682 .106402522$

C $1.188650804 \quad 0.3244903820 .905896578$

S $0.490519318-1.825802264-0.874711164$

C $1.214589452-1.119924225-2.417325852$

C $1.503867891-2.227507426-3.438057247$

C 2.611182998 -3.201220749 -3.019419966

C $-2.292200605-1.4608195472 .614756539$

O $2.334527487 \quad 0.493828820 \quad 0.090328086$

C $3.0975844381 .660469867 \quad 0.363231383$

O $2.484574156-0.0067012502 .906966559$

C $3.310474767-0.952140083 \quad 3.563929145$

O

C $0.158590314 \quad 0.739784434 \quad 4.745388670$

O

C $-4.414593122-2.429017617 \quad 2.332426352$

H $\quad-0.2206090550 .472737821 \quad-0.727282939$

H $\quad-0.634145580-2.3317733351 .589558999$

H $0.311236397-1.5444800363 .690657313$

H $1.799086766-1.5578825251 .716763546$

H $\quad 0.831662578 \quad 1.293620008 \quad 1.281854308$

H $0.493813311-0.406704917$ - 2.831883243

H 2.125054348 -0.579747337 -2.150588779

H $\quad 1.788264621-1.736217042$-4.377997951

H $0.579777611-2.779703753$-3.649307654

H $3.549921274-2.669370504-2.827222059$

H 2.798764440 -3.938919528 -3.806327212

H 2.345777069 -3.743576527 -2.106968666
H $\quad-2.160301470 \quad-1.860959151 \quad 3.635280572$

H $\quad-2.740935616 \quad-0.461733518 \quad 2.703649443$

H $3.509069164 \quad 1.645810276 \quad 1.377264759$

H $3.914533443 \quad 1.676495863$-0.363165390

H $4.073316729 \quad-0.388334424 \quad 4.106490101$

H $3.805424433-1.6217985232 .845898873$

H $\quad-0.436061456 \quad 1.5834191835 .106476995$

H $1.214403561 \quad 1.025759094 \quad 4.712112249$

H $\quad-4.929594524 \quad-1.4551486392 .333548811$

H $\quad-4.442272281 \quad-2.831366063 \quad 3.358865441$

H $2.4958640652 .573454188 \quad 0.235860525$

H 2.753153995 -1.566843839 4.286159077

H $\quad 0.037631275 \quad-0.098738070 \quad 5.449574230$

H $\quad-4.954554423 \quad-3.112676927 \quad 1.672753561$

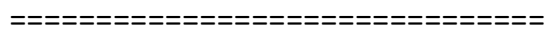

4- $\beta$ (MeO)

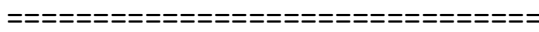

C $0.239081891-0.000016926-0.053197858$

$\begin{array}{llll}\text { O } & -1.057650011 & 0.103913229 & 0.512227489\end{array}$

C $-1.360378153 \quad-0.8804354491 .493031422$

C $-0.376348632 \quad-0.772441057 \quad 2.676825851$

C $1.032468323-1.0489930222 .115268069$

C $1.364561767-0.0382826581 .009898927$

S $0.420741817 \quad 1.356084785-1.264568866$

C $0.0250503602 .812908351 \quad-0.205173318$

C $-0.474950403 \quad 3.972768861-1.071061872$

C $\quad-0.777980832 \quad 5.223156007 \quad-0.237609476$

C $-2.849368368-0.783378272 \quad 1.801791248$

O $2.549076171 \quad-0.4331874820 .339886662$

C 3.7081264850 .3055733570 .688734783

O $2.041712626-0.956312513 \quad 3.106966017$

C $2.244623366-2.1426166253 .849165249$

O

C -0.8955475010 .6166390934 .555644939$

O 
C $-4.577163885 \quad 0.613412418 \quad 2.553063261$

H $0.328972031-0.915939317-0.658780553$

H -1.224134633 -1.890527403 1.059175056

H $\quad-0.634948698$-1.535009347 3.427421026

H 1.047225703 -2.054700264 1.662332814

H $1.469676527 \quad 0.947639325 \quad 1.475573976$

H $\quad-0.7391348542 .4961558720 .509463859$

H $\quad 0.919753934 \quad 3.113764011 \quad 0.350270954$

H $\quad-1.373492638 \quad 3.654366431$-1.612122478

H $0.276750762 \quad 4.213433923-1.833870762$

H $\quad-1.549001378 \quad 5.021047251 \quad 0.514222421$

H $\quad-1.136204362 \quad 6.039949010-0.872619657$

H $\quad 0.114445798 \quad 5.578704605 \quad 0.289739198$

H $\quad-3.389083230-0.8721596530 .845325685$

H $\quad$-3.136999637 -1.6474724552 .426869968$

H $4.526900287-0.0897304010 .081478003$

H $3.953907776 \quad 0.191011641 \quad 1.751060502$

H 3.065761081 -1.949147801 4.543801296

H 2.521378254 -2.986777389 3.199063606

H $\quad-0.752450949 \quad 1.651267858 \quad 4.878343176$

H $\quad-0.365258804-0.043861835 \quad 5.258529665$

H $\quad-4.738230574 \quad 1.574274534 \quad 3.047595750$

H $\quad-5.052428932-0.1796565573 .153306956$

H $3.587953076 \quad 1.374238213 \quad 0.458266460$

H $1.358409960-2.4326594194 .433796103$

H $\quad-1.9684124490 .3892958334 .572905005$

H $\quad \begin{array}{llll}-5.064175310 & 0.634486643 & 1.565596923\end{array}$

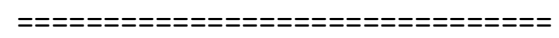

$10-\alpha(\mathrm{MeO}, \mathrm{Ph})$

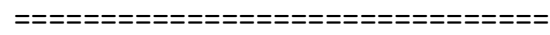

C $\quad-0.030483024-0.175231402 \quad 0.397786757$

O

C $-0.891981148-1.440122250 \quad 2.272353776$

C $0.343806391-1.118802122 \quad 3.122755230$

C $1.569746614-0.992629585 \quad 2.183294302$
C $\quad \begin{array}{llll}1.288952944 & 0.103877132 & 1.145898658\end{array}$

S $0.181379259-1.575442878-0.898287360$

C $0.695889687-0.586320140-2.385882530$

C $1.593313965-1.384682804-3.338603727$

C $2.982656902-1.706235976-2.780976418$

C $-2.189056427-1.463939736 \quad 3.065155812$

O $2.311111875 \quad 0.2406846320 .183633024$

C 3.3020955591 .2278091190 .484921102

O $2.753380534 \quad-0.6472830192 .879189098$

C $3.491196573-1.756022845 \quad 3.378153134$

O $0.0811907910 .083635597 \quad 3.821808178$

C $0.751541393 \quad 0.240833616 \quad 5.071336106$

O

C $-4.518823742 \quad-1.7861643792 .790356396$

Bi -2.479071791 -2.915494554 -1.491764473

H $\quad-0.3128231520 .691042833-0.204573854$

H $\quad-0.748142297 \quad-2.417727636 \quad 1.790683299$

H 0.503233109 -1.950791534 3.828093237

H $\quad 1.714118005$-1.947172684 1.651351657

H $1.127220528 \quad 1.045065641 \quad 1.691042756$

H $\quad-0.210630133 \quad-0.231303831-2.883438233$

H $1.237861228 \quad 0.266874916$-1.973521484

H 1.693538607 -0.775343821 -4.246672424

H 1.084998208 -2.303429472 -3.650707723

H $3.530576806-0.791986864-2.532136743$

H $3.570309254-2.261147352$-3.518546386

H $2.923376303-2.311821365-1.871810785$

H $-2.087249944 \quad-2.1720765353 .904136609$

H $-2.371921036 \quad-0.465057623 \quad 3.482209911$

H $3.843515747 \quad 0.978089823 \quad 1.400216090$

H $3.990843896 \quad 1.239748868$-0.362284855

H 4.384262666 -1.350325148 3.857102648

H $3.794934916 \quad-2.430359672 \quad 2.565231348$

H $0.318765698 \quad 1.125717322 \quad 5.543153903$

H 1.8272027790 .3850970694 .938413995 
H $\quad-4.747795485-0.7627995803 .120045086$

H $\quad-4.591743254 \quad-2.4562292003 .659503590$

O -4.241036505 -4.055472095 -2.080716683

S $-5.668639520-3.856877780-1.459491075$

O $\quad-5.536567478-3.305578606-0.101167674$

C $-6.166931687-5.643311041-1.263347763$

F $-6.182840565-6.244316482-2.448155002$

F $-5.293314531-6.261485642-0.459746362$

F -7.378261835 -5.685402371 -0.715370327

O $-6.588559009-3.249021467-2.408936534$

H $2.847526327 \quad 2.2227299010 .590921974$

H 2.925320568 -2.332091387 4.123364388

H $0.578878842 \quad-0.627382748 \quad 5.724113569$

H $\quad-5.238180519-2.0958754492 .031650581$

C -3.569495795 -1.006580495-1.667011999

C $-4.307363449-0.865357859-2.845522672$

C $-3.544892156-0.040223767-0.665414275$

C $\quad \begin{array}{llll}-5.025181601 & 0.318935642 & -3.030799931\end{array}$

C $-4.2762169391 .132415846-0.877026166$

C $-5.004930136 \quad 1.313959812-2.053351849$

H $\quad-4.367241023$-1.651867642 -3.590214672

H $\quad-3.005726991 \quad-0.1973709130 .261206042$

H $\quad$-5.611350655 0.446706683 -3.935694345

H $\quad$-4.282199935 1.897724595 -0.106436886

H $\quad-5.572495484 \quad 2.227532154$-2.202597232

C $-1.641893180-3.523443413-3.415086613$

C $-1.243214681-4.853550950-3.584764795$

C $-1.623370826-2.623206635$-4.486096728

C $-0.810504901-5.279417662-4.842281537$

C $-1.193592288-3.064054612-5.739794811$

C $-0.786274505-4.387400344-5.915669012$

H $-1.277212232-5.562547067-2.762689302$

H $-1.946351726-1.593318108$-4.363874530

H $\quad$-0.501639956 -6.311085827 -4.981842910

H $\quad-1.182997609-2.372935265$-6.577358087
H $\quad-0.455463886-4.726108596-6.892777467$

C $-1.900485607 \quad-4.366669210 \quad 0.070124181$

C -2.845894768 -4.809607420 0.995173509

C $-0.603233815-4.8852337020 .020300132$

C $-2.466270693 \quad-5.8062598951 .899094964$

C $-0.248246072 \quad-5.8822695190 .934666768$

C $-1.176463184-6.339903797 \quad 1.871481761$

H -3.847785475 -4.399247189 1.020268802

H $\quad 0.127203972$-4.535217202 -0.701666300

H $\quad-3.190680541 \quad-6.1650053112 .624316498$

H $0.753714262-6.3001772010 .903949678$

H $\quad-0.895423745 \quad-7.115598134 \quad 2.577385725$

$10-\beta(\mathrm{MeO}, \mathrm{Ph})$

C $0.059575814 \quad-0.0469625390 .392141946$

O

C $-1.018283811 \quad-1.220126218 \quad 2.177139679$

C $0.259581199-1.1458286163 .032806805$

C $1.465801270-1.2909771052 .079215247$

C $1.448751603-0.130641410 \quad 1.068457584$

S $-0.1226635521 .496557889-0.603331837$

C $\quad \begin{array}{llll}0.047260460 & 2.792708647 & 0.716751425\end{array}$

C $-0.422886064 \quad 4.1764715990 .181333686$

C $-0.270887676 \quad 5.250096113 \quad 1.266953799$

C -2.349324069 -1.264170251 2.915952513

O $2.361914096 \quad-0.332128746 \quad 0.012591722$

C 3.6413949590 .2755579350 .181793106

O $2.702699752-1.2381767512 .757831915$

C $3.150150642-2.4845841413 .273567838$

O $0.3669556320 .105596423 \quad 3.681704730$

C $0.338425710 \quad 0.066695337 \quad 5.100184269$

O

C $-3.783902918-0.1601044314 .412131832$

Bi $\quad-2.913563142 \quad 1.148553263$-1.698666741 
H $0.013509885-0.848661204-0.358875743$

H $\quad-0.977494534-2.1530705791 .588501759$

H $0.254127684-1.975486145 \quad 3.754251462$

H $\quad 1.378170887$-2.236669576 1.518453760

H $1.666712333 \quad 0.783454231 \quad 1.632496493$

H $\quad-0.694166612 \quad 2.475484934 \quad 1.536685438$

H $0.986554272 \quad 2.798496933 \quad 1.072050361$

H $\quad-1.4594210464 .169494791$-0.176204827

H $0.2188414694 .427900541 \quad-0.672533684$

H $\quad-0.920311835 \quad 5.041950324 \quad 2.123344257$

H $\quad-0.540632602 \quad 6.2343170090 .873320558$

H $\quad 0.760578151 \quad 5.307031871 \quad 1.629816263$

H $\quad-3.157692469-1.3858654652 .175211377$

H $\quad-2.353066627 \quad-2.168230413 \quad 3.548923192$

H $4.2045956330 .064761329-0.729757681$

H $4.168117719-0.1344899261 .047826265$

H 4.123541466 -2.303211842 3.733299069

H 3.264158870 -3.232136972 2.475588863

H $0.494136596 \quad 1.091821018 \quad 5.443974134$

H 1.142874858 -0.563837182 5.502099441

H $\quad-3.8516774990 .757500323 \quad 5.000552675$

H $\quad-3.810725722-1.0214727515 .095254943$

O

S $-5.915139073-0.079428130-2.264971789$

O $-6.014701620-1.213575303-3.170927817$

C $\quad-7.4378696790 .968986861-2.515388928$

F -7.330792855 2.098444447 -1.807675506

F $\quad-7.571731457 \quad 1.263368076$-3.804952960

F $\quad-8.4932822650 .279299035$-2.094379041

O

H $3.551493615 \quad 1.365520504 \quad 0.297684391$

H 2.471876750 -2.887219492 4.038929170

H $\quad-0.631255994-0.2857685645 .472735882$

H $\quad-4.650461657 \quad-0.221157963 \quad 3.737449103$

C $-3.8152423112 .344753076-0.083174535$
C $-4.7181784853 .323753545-0.504131261$

C $\quad \begin{array}{llll}3.500849244 & 2.130544816 & 1.257942668\end{array}$

C $\quad-5.3168353894 .129507690 \quad 0.467340652$

C $-4.1179242392 .949344864 \quad 2.209230858$

C $\quad \begin{array}{llll}-5.015060390 & 3.943991361 & 1.817350542\end{array}$

H $\quad-4.980074212 \quad 3.451861815$-1.549101762

H $\quad-2.808918297 \quad 1.359683306 \quad 1.579192131$

H $\quad-6.023694816 \quad 4.8951891120 .161822922$

H $\quad-3.888137471 \quad 2.798715496 \quad 3.259571045$

H $\quad-5.486348564 \quad 4.573396134 \quad 2.566364457$

C $-2.411156536-0.989356334-1.818380749$

C -3.104227327 -1.955455168 -1.086476450

C -1.405529040 -1.325701904 -2.729883259

C $-2.768381115-3.298327377-1.279647568$

C $-1.085744457-2.675061071-2.906110527$

C $-1.765613021-3.655976046-2.182813928$

H $\quad-3.913288705-1.686697945-0.416542420$

H $\quad-0.875986169-0.569520891-3.303248545$

H $\quad-3.309648796$-4.064066173 -0.731693292

H $\quad-0.311280371 \quad-2.952349073-3.614858129$

H -1.520339775 -4.703393026 -2.330871025

C $-2.251232658 \quad 2.193177058$-3.510694355

C $-1.448067238 \quad 3.333362465$-3.446330153

C $-2.693460340 \quad 1.676535958$-4.733937466

C $-1.083463033 \quad 3.971358617-4.634263572$

C $-2.310323317 \quad 2.321830229-5.911909885$

C $-1.511108757 \quad 3.464707580-5.862216966$

H $\quad-1.108702367 \quad 3.735189759-2.498155658$

H $\quad-3.3426188750 .808819662$-4.779331665

H $\quad-0.466009528 \quad 4.863732463 \quad-4.595356540$

H $\quad-2.648402357 \quad 1.930486651$-6.866749710

H $\quad-1.223548103 \quad 3.963793955$-6.782654575

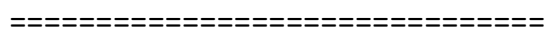

$10-\alpha(\mathrm{MeO}, \mathrm{Me})$

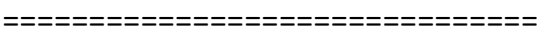


C $\quad-0.072688208-0.3365068340 .120513598$

$\begin{array}{llll}0 & -1.153766247 & -0.585234597 & 0.943934695\end{array}$

C $-0.917268580-1.370755946 \quad 2.137165031$

C $0.233013183-0.7903730442 .968801662$

C $1.497927113-0.7106423222 .079725066$

C 1.1799550810 .1702957860 .865811060

S $0.290886414-1.908711576-0.933474737$

C $0.985679082-1.169789044-2.493845818$

C $1.966752616-2.110212367-3.203198221$

C $3.257786821-2.386656058-2.427617092$

C $-2.238352661-1.4001639392 .886556516$

O $2.237939003 \quad 0.248308499-0.063471605$

C $3.130231528 \quad 1.354538806 \quad 0.120478419$

$\begin{array}{llll}\text { O } & 2.602714454 & -0.141965443 & 2.754146097\end{array}$

C $3.391681683-1.0704863093 .490785931$

$\begin{array}{llll}\text { O } & -0.177863549 & 0.481665515 & 3.429542511\end{array}$

C $0.397983558 \quad 0.918970246 \quad 4.661125195$

$\begin{array}{llll}0 & -3.186318986 & -2.102486363 & 2.100563855\end{array}$

C $-4.467067621-2.186907652 \quad 2.730456223$

Bi -2.406030459 -2.996448554 -1.608699130

C $-2.152762388-4.4367041680 .036257528$

C $-3.333184682-1.013283815-1.344907854$

C -1.516947255 $-3.582708837-3.537948278$

H $\quad-0.3846564220 .405653068-0.618269588$

H $\quad-0.650141883-2.396120037 \quad 1.839192325$

H $\quad 0.419271322-1.471446318 \quad 3.814944420$

H $\quad 1.754038110-1.7224174001 .724489430$

H 0.9101792391 .1671444291 .243847761

H $\quad 0.151292517-0.891155249-3.143894383$

H $1.494750332-0.265781976-2.157247362$

H $2.206920971-1.629869617-4.160622192$

H $1.471760003-3.054638045-3.461212114$

H $3.786319889-1.456609020-2.197109032$

H $3.927555181-3.018338417-3.018115366$

H $3.060165659-2.899048406-1.481428165$
H $\quad-2.087440342-1.900115004 \quad 3.856743159$
H $-2.565725595-0.371221427 \quad 3.081359932$
H $3.6405608721 .291482108 \quad 1.083790433$
H $3.858585015 \quad 1.298674592-0.690813831$
H $4.225218094-0.5072712883 .913823968$
H $3.786520670-1.8616611142 .838697687$
H -0.1382763241 .8268798864 .944645466$
H $\quad 1.463073125 \quad 1.140249347 \quad 4.552412772$
H $-4.873075958-1.185958892 \quad 2.928538555$
H $\quad-4.402256143 \quad-2.735954753 \quad 3.680089631$
o $-4.208029774-3.894216658-2.430337752$
S $-5.544891464-3.868823344-1.600393181$
o $-5.201540328-3.508607204-0.214723263$
C $-5.954028428-5.688220699-1.598093336$
F $-6.154436469-6.108664689-2.840184103$
F $-4.927139146-6.363309316-1.057365725$
F $-7.043492246-5.879626447-0.865344251$
o $-6.602609252-3.166205480-2.305686640$
H 2.5905972162 .3089628960 .053761790
H $2.829272304-1.534691825 \quad 4.312171580$
H $\quad 0.260078177 \quad 0.165527283 \quad 5.449867104$
H $\quad-5.122645575 \quad-2.7168029202 .040334860$
H $-0.641108738-4.199968408-3.339111035$
H $-1.246913861-2.692225136-4.104391214$
H $-2.301066345-4.146565956-4.043112650$
H $-3.451304978-0.904323634-0.267429216$
H $-4.284183900-1.053740815-1.875775225$
H $-2.660070395-0.265986010-1.762577158$
H $-2.789887552-5.282726730-0.220786273$
H $\quad-2.502265645 \quad-3.9249499870 .934493089$
H $-1.097902849-4.7079080380 .079338900$

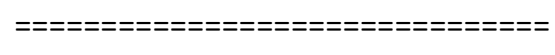
$10-\beta(\mathrm{MeO}, \mathrm{Me})$

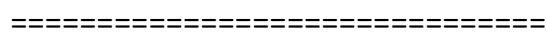
$\begin{array}{llll}\text { C } & 0.104314588 & -0.031415078 & 0.062153191\end{array}$ 
O

C $-1.329806194-1.055718758 \quad 1.700106017$

C $-0.301936297-0.7519407012 .802633760$

C $1.105755535-0.940927275 \quad 2.192424625$

C 1.2891091290 .0763244601 .049995859

S $0.079530458 \quad 1.361847532-1.162803836$

C $0.241515234 \quad 2.861707170-0.092681729$

C $-0.0491063614 .141188897-0.884298015$

C $0.238809274 \quad 5.392787636-0.044221035$

C $-2.798571226-1.104926868 \quad 2.100989470$

O $2.428174528-0.171514098 \quad 0.259174019$

C 3.6423485610 .4419259890 .695831767

O $2.135385465-0.707788244 \quad 3.127179940$

C $2.469552801-1.8293281753 .936846747$

O

C $-0.808891817 \quad 0.755277792 \quad 4.599975397$

O

C $-4.6126491210 .100900582 \quad 2.994766093$

Bi $-2.763385654 \quad 1.120009133$-1.859001620

C $-3.3945535692 .166983054-0.020882007$

C $-2.4186704372 .383212530-3.630230686$

C $-2.334108924-1.003804730-2.265986358$

H $0.291095518-0.916847334-0.564850981$

H -1.099145943 -2.055004476 1.292604883

H $\quad-0.447292625$-1.464605355 3.626588228

H $1.192059286-1.9573356531 .772616496$

H $1.320896177 \quad 1.065537646 \quad 1.520802512$

H $\quad-0.415377420 \quad 2.738359813 \quad 0.772507084$

H $\quad 1.2774804772 .870113555 \quad 0.254588816$

H $-1.097381184 \quad 4.155984824-1.210289824$

H $0.5637694414 .158206480-1.793637356$

H $\quad-0.369212146 \quad 5.414277572 \quad 0.866156139$

H $0.016885661 \quad 6.297213158$-0.617325595

H $\quad 1.291633126 \quad 5.435549845 \quad 0.251864337$

H $\quad-3.401413350 \quad-1.412076636 \quad 1.232127012$
H $-2.907345142 \quad-1.8921715892 .866032181$

H $4.386481223 \quad 0.221938915$-0.071853289

H $3.968713761 \quad 0.045105629 \quad 1.659713413$

H $3.300807550-1.5194055664 .572431028$

H 2.784552719 -2.686182549 3.324938322

H $\quad-0.775924523 \quad 1.8279468994 .802613460$

H $\quad-0.121112604 \quad 0.246702297 \quad 5.288212799$

H -4.8634690601 .0962477363 .366449866$

H $-4.767668003 \quad-0.6302174413 .800257472$

$\begin{array}{lllll}\text { O } & -4.818413227 & 0.885983802 & -2.589364498\end{array}$

S $-5.711725838-0.185949066-1.871816407$

O $-6.131367161-1.245578176-2.772908042$

C $-7.209343870 \quad 0.845020562-1.454881863$

F $-6.827608146 \quad 1.877789034-0.686743550$

F $-7.767001521 \quad 1.302871109-2.566954363$

F $\quad-8.070520844 \quad 0.091126107 \quad-0.782639910$

O

H $3.528172448 \quad 1.5323879590 .779975694$

H $1.635360821-2.1417924374 .579754874$

H $\quad-1.8322444290 .396835432 \quad 4.762020830$

H $\quad-5.268235829-0.153461815 \quad 2.151524141$

H $\quad-3.032131905$-1.290018330 -3.053827237

H $\quad-2.528630572-1.554091000$-1.348853207

H $\quad-1.297959826-1.088820260-2.592658407$

H $-1.824049643 \quad 1.810940080$-4.343696863

H $-1.895760454 \quad 3.293577970$-3.339553465

H $-3.408234282 \quad 2.599032151$-4.034561277

H $-2.849504172 \quad 3.1104820850 .003311753$

H $\quad-3.156251537 \quad 1.522822146 \quad 0.827745356$

H $\quad-4.465577704 \quad 2.316258230 \quad-0.149208239$ 


\section{Vibrational Frequencies of Optimized Structures}

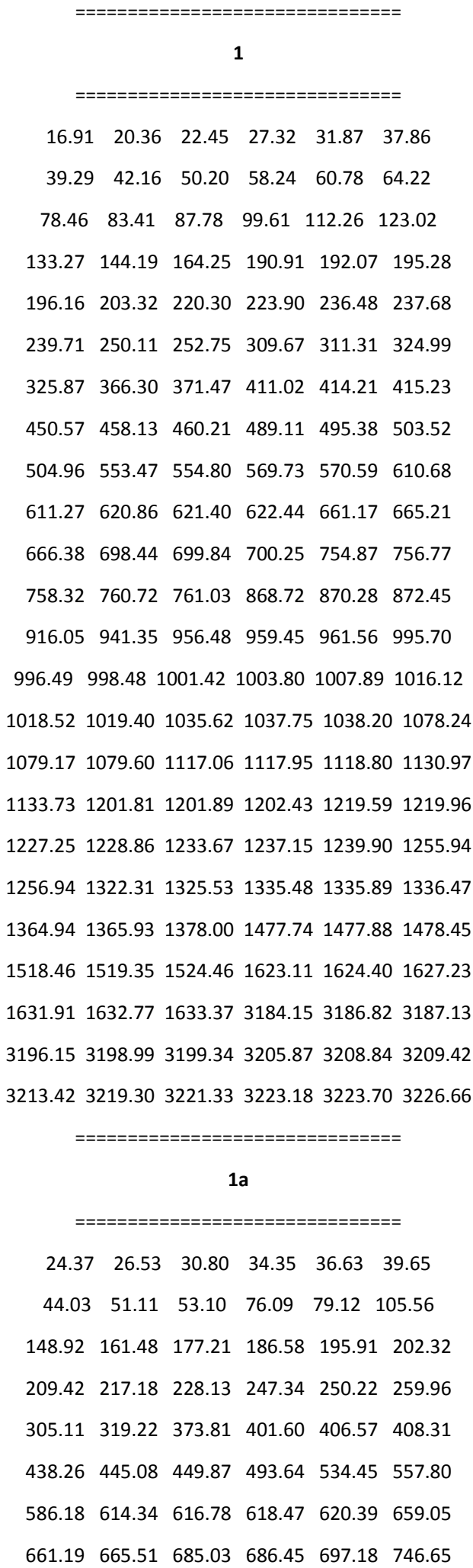

$\begin{array}{llllll}16.91 & 20.36 & 22.45 & 27.32 & 31.87 & 37.86\end{array}$

$\begin{array}{llllll}39.29 & 42.16 & 50.20 & 58.24 & 60.78 & 64.22\end{array}$

$\begin{array}{llllll}333.27 & 144.19 & 164.25 & 190.91 & 192.07 & 195.28\end{array}$

$\begin{array}{lllllll}239.71 & 250.11 & 252.75 & 309.67 & 311.31 & 324.99\end{array}$

$\begin{array}{llllll}325.87 & 366.30 & 371.47 & 411.02 & 414.21 & 415.23\end{array}$

$\begin{array}{llllll}450.57 & 458.13 & 460.21 & 489.11 & 495.38 & 503.52\end{array}$

$\begin{array}{llllll}611.27 & 620.86 & 621.40 & 622.44 & 661.17 & 665.21\end{array}$

$\begin{array}{lllllll}666.38 & 698.44 & 699.84 & 700.25 & 754.87 & 756.77\end{array}$

$\begin{array}{llllll}916.05 & 941.35 & 956.48 & 959.45 & 961.56 & 995.70\end{array}$

996.49998 .481001 .421003 .801007 .891016 .12

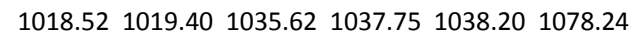

1133.731201 .811201 .891202 .431219 .591219 .96

$\begin{array}{llllll}1227.25 & 1228.86 & 1233.67 & 1237.15 & 1239.90 & 1255.94\end{array}$

$\begin{array}{llllll}1256.94 & 1322.31 & 1325.53 & 1335.48 & 1335.89 & 1336.47\end{array}$

$\begin{array}{llllll}1364.94 & 1365.93 & 1378.00 & 1477.74 & 1477.88 & 1478.45\end{array}$

$\begin{array}{llllll}1518.46 & 1519.35 & 1524.46 & 1623.11 & 1624.40 & 1627.23\end{array}$

$1631.911632 .771633 .373184 .153186 .82 \quad 3187.13$

3196.153198 .993199 .343205 .873208 .843209 .42

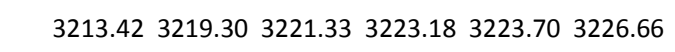
$\begin{array}{lllll}661.19 & 665.51 \quad 685.03 \quad 686.45 & 697.18 & 746.65\end{array}$ $\begin{array}{llllll}748.93 & 750.04 & 760.96 & 829.35 & 849.78 & 853.70\end{array}$ $\begin{array}{llllll}859.64 & 937.90 & 942.59 & 944.73 & 987.01 & 988.83\end{array}$ $\begin{array}{llllll}989.95 & 990.35 & 994.78 & 1003.12 & 1027.55 & 1028.07\end{array}$

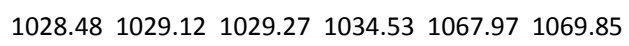
$1077.94 \quad 1084.081112 .841114 .531117 .551193 .38$ $\begin{array}{llllll}1206.85 & 1207.27 & 1207.32 & 1222.99 & 1223.94 & 1226.27\end{array}$ $\begin{array}{llllll}1270.19 & 1296.73 & 1332.86 & 1335.32 & 1338.40 & 1354.45\end{array}$ $\begin{array}{llllll}1360.21 & 1361.64 & 1363.25 & 1474.55 & 1475.71 & 1478.50\end{array}$ $\begin{array}{lllll}1508.35 & 1510.051515 .31 & 1610.661613 .94 & 1621.12\end{array}$ $1623.91 \quad 1626.121627 .093173 .863185 .893190 .03$ 3198.983201 .173201 .793206 .763209 .883210 .25 $3214.993220 .33 \quad 3220.613221 .363233 .703239 .44$

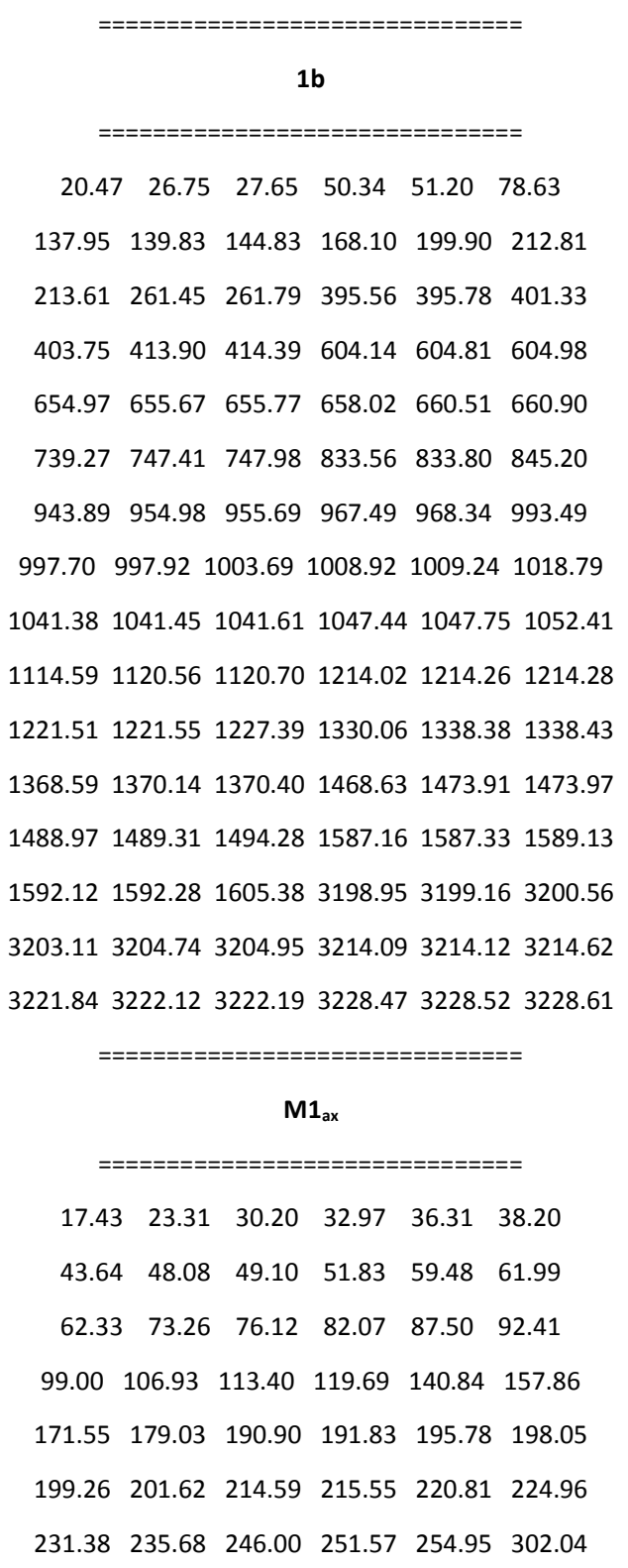

\author{
$\begin{array}{llllll}231.38 & 235.68 & 246.00 & 251.57 & 254.95 & 302.04\end{array}$
}




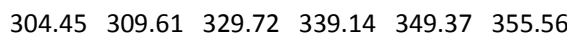

$404.66 \quad 415.76 \quad 417.19 \quad 458.54 \quad 459.56 \quad 466.83$

$\begin{array}{llllll}493.75 & 496.07 & 501.32 & 522.35 & 555.97 & 558.35\end{array}$

$\begin{array}{llllll}562.51 & 576.36 & 606.50 & 616.23 & 618.72 & 620.36\end{array}$

$\begin{array}{llllll}625.88 & 655.25 & 659.38 & 664.81 & 669.77 & 691.12\end{array}$ $\begin{array}{llllll}694.58 & 707.97 & 725.49 & 746.69 & 750.19 & 754.00\end{array}$

$\begin{array}{llllll}759.07 & 761.14 & 852.93 & 856.43 & 864.66 & 892.89\end{array}$

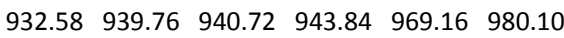
$986.12987 .53990 .28993 .81 \quad 1001.93 \quad 1009.00$ 1013.351015 .181015 .671019 .461034 .321034 .36 $1037.98 \quad 1064.291070 .41 \quad 1075.801086 .661110 .17$

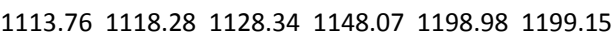
$1199.301208 .791216 .14 \quad 1222.41 \quad 1225.71 \quad 1227.79$

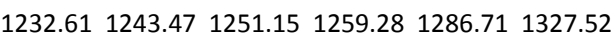

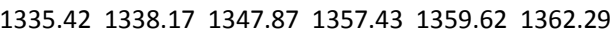
$1369.47 \quad 1396.591469 .33 \quad 1471.301474 .15 \quad 1477.46$ $1482.681491 .23 \quad 1499.32 \quad 1513.57 \quad 1515.05 \quad 1520.71$

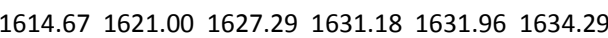
3067.963070 .203166 .413169 .853171 .023174 .27 3180.603182 .243183 .893184 .573189 .473192 .06 $3195.393199 .803201 .43 \quad 3207.343209 .143209 .39$ 3222.233241 .753261 .14

\begin{tabular}{rrrrrr}
\multicolumn{1}{c}{ S1 } \\
\multicolumn{1}{c}{$========================$} \\
15.52 & 20.81 & 25.94 & 26.85 & 31.88 & 37.02 \\
42.43 & 44.96 & 46.74 & 47.31 & 50.56 & 56.02 \\
60.54 & 62.62 & 69.90 & 74.52 & 82.72 & 84.80 \\
90.61 & 94.06 & 104.28 & 109.49 & 126.73 & 129.86 \\
138.84 & 162.49 & 168.99 & 177.34 & 183.92 & 187.68 \\
194.08 & 199.44 & 210.72 & 214.29 & 221.98 & 226.62 \\
233.11 & 236.00 & 243.02 & 248.35 & 268.14 & 297.54 \\
305.70 & 310.78 & 328.85 & 334.30 & 351.76 & 376.35 \\
379.41 & 406.41 & 408.23 & 413.93 & 459.62 & 466.31 \\
470.09 & 486.37 & 493.04 & 499.90 & 504.55 & 553.97 \\
554.21 & 565.84 & 568.65 & 609.58 & 613.78 & 623.56 \\
624.05 & 628.00 & 653.89 & 658.69 & 664.15 & 703.30 \\
704.64 & 707.86 & 729.51 & 741.82 & 744.75 & 749.37 \\
754.03 & 755.75 & 756.12 & 857.67 & 858.93 & 864.10 \\
866.54 & 909.44 & 923.56 & 930.39 & 936.11 & 946.74 \\
963.16 & 974.49 & 981.42 & 987.07 & 991.97 & 994.60
\end{tabular}

999.161000 .161006 .361011 .391035 .091038 .06 1040.361042 .991076 .161081 .491082 .571085 .92 $1107.021108 .63 \quad 1109.351116 .681144 .661152 .45$ 1192.831192 .991193 .201207 .951215 .071221 .76 $1222.31 \quad 1225.04 \quad 1228.46 \quad 1232.501234 .96 \quad 1237.87$

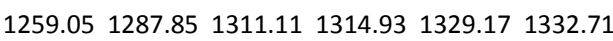

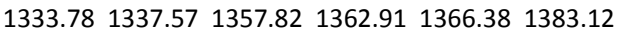

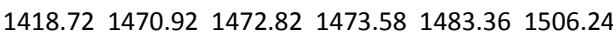

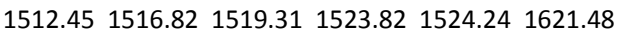
$1625.181630 .94 \quad 1631.851632 .68 \quad 1634.30 \quad 3023.18$ 3034.393044 .923082 .943113 .653121 .973134 .35 3156.843159 .663162 .533170 .353173 .433176 .01 3182.833186 .443186 .673191 .813196 .043202 .70 3208.593211 .973218 .00

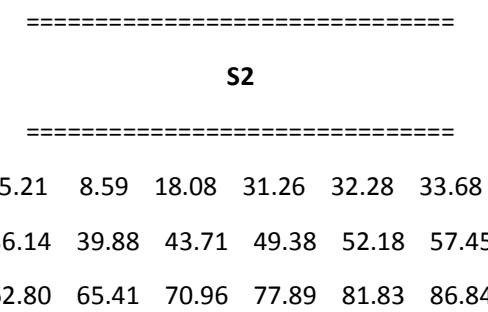

$\begin{array}{llllll}91.67 & 102.44 & 105.87 & 110.37 & 121.76 & 132.11\end{array}$ $\begin{array}{llllll}152.83 & 172.57 & 184.50 & 187.90 & 194.65 & 195.69\end{array}$ $\begin{array}{llllll}197.20 & 199.15 & 215.22 & 220.70 & 222.71 & 226.77\end{array}$ $\begin{array}{llllll}235.02 & 240.30 & 245.84 & 250.01 & 269.37 & 300.62\end{array}$ $\begin{array}{llllll}304.22 & 305.51 & 329.42 & 335.40 & 345.78 & 349.64\end{array}$ $\begin{array}{llllll}372.69 & 404.80 & 406.21 & 411.22 & 459.20 & 466.06\end{array}$ $\begin{array}{llllll}471.56 & 486.63 & 496.49 & 497.78 & 502.38 & 553.79\end{array}$ $\begin{array}{llllll}555.35 & 558.38 & 565.27 & 610.55 & 616.12 & 621.64\end{array}$ $\begin{array}{llllll}623.10 & 628.15 & 659.94 & 661.50 & 668.68 & 689.90\end{array}$ $\begin{array}{llllll}696.34 & 710.34 & 712.43 & 743.06 & 745.44 & 746.51\end{array}$ $\begin{array}{llllll}747.73 & 752.64 & 756.89 & 843.76 & 850.02 \quad 865.70\end{array}$

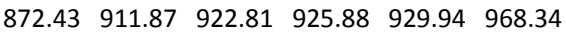

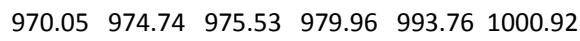
1002.111004 .561005 .921012 .661033 .371036 .28 1041.591044 .101076 .711079 .941089 .971091 .51 $1106.001111 .921112 .03 \quad 1119.251147 .231162 .79$ 1182.521192 .781192 .941193 .591207 .701211 .69 $1222.13 \quad 1222.991225 .301228 .601231 .341242 .20$

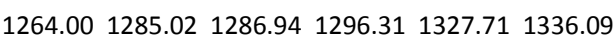
$1336.571339 .381361 .21 \quad 1361.97 \quad 1362.271385 .02$

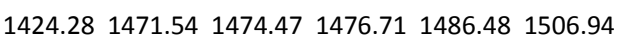




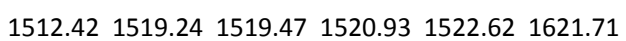
1624.861629 .281633 .251634 .641635 .713029 .51 3043.863050 .993094 .253115 .933125 .453147 .02 3158.723163 .193163 .993169 .223176 .783177 .67 3180.293186 .693190 .083190 .513194 .753199 .78 3201.823232 .593254 .86

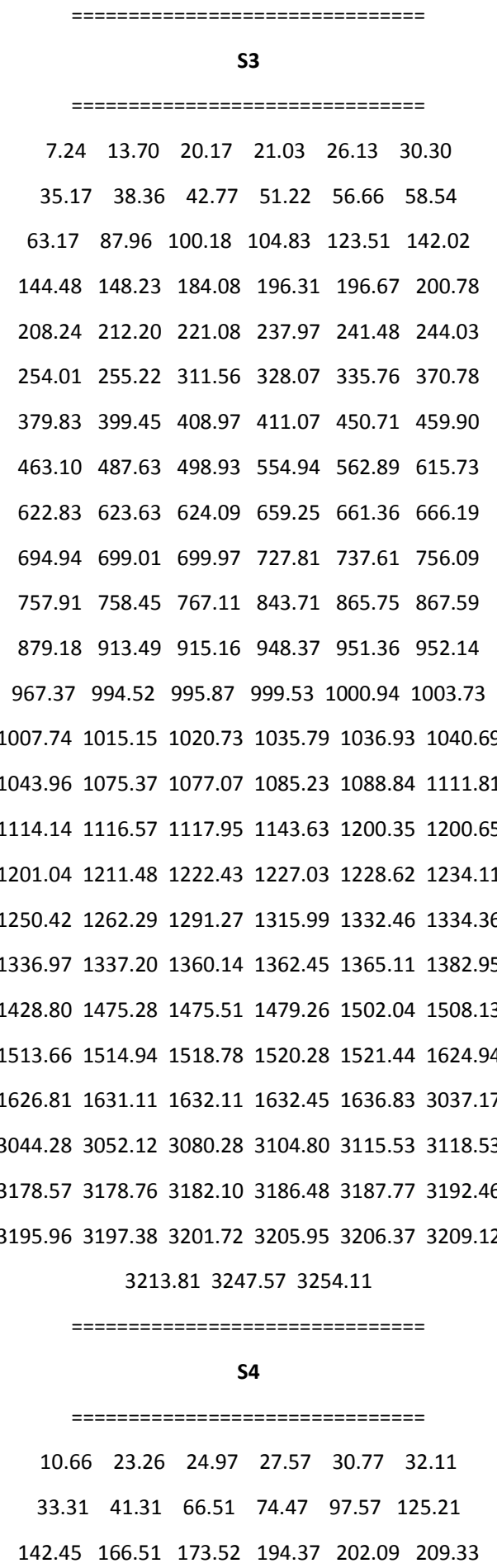

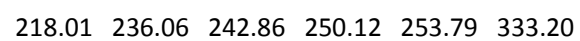
$\begin{array}{llllll}387.38 & 403.01 & 403.72 & 406.78 & 443.75 & 445.92\end{array}$ $\begin{array}{llllll}452.07 & 619.98 & 620.65 & 620.92 & 662.44 & 665.66\end{array}$ $\begin{array}{llllll}666.03 & 696.31 & 696.80 & 697.77 & 705.02 & 746.53\end{array}$ $\begin{array}{llllll}747.09 & 748.29 & 769.23 & 855.88 & 856.81 & 860.59\end{array}$ $\begin{array}{llllll}884.30 & 907.74 & 935.15 & 935.78 & 938.10 & 984.11\end{array}$ $984.95987 .65999 .76 \quad 1000.24 \quad 1004.871024 .55$ 1024.721025 .321032 .691034 .641034 .991037 .50

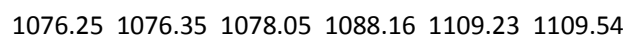

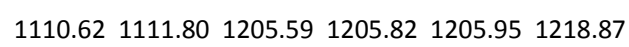

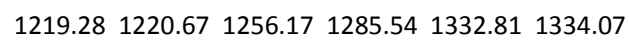

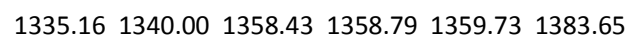
$\begin{array}{llllll}1432.70 & 1475.27 & 1476.54 & 1478.23 & 1496.42 & 1507.92\end{array}$

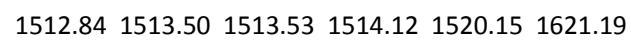
$\begin{array}{llllll}1621.37 & 1623.33 & 1627.51 & 1628.35 & 1629.08 & 3044.93\end{array}$ 3050.983062 .723087 .703114 .793128 .663131 .63 3181.183182 .973183 .483183 .663184 .853187 .04 3200.343200 .583201 .263209 .543209 .793210 .65 3219.483219 .613220 .18

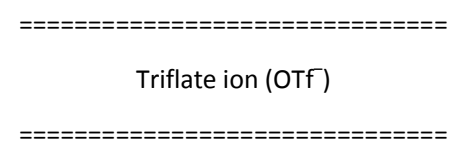

$\begin{array}{llllll}56.66 & 189.98 & 190.73 & 288.59 & 330.26 & 330.63\end{array}$ $\begin{array}{llllll}498.40 & 498.72 & 555.37 & 555.68 & 621.03 & 739.17\end{array}$ 999.041179 .691179 .861225 .861256 .301256 .51

$\mathrm{Me}_{2} \mathrm{~S}$

$\begin{array}{llllll}187.74 & 189.47 & 263.52 & 684.29 & 734.83 & 920.15\end{array}$ $960.32 \quad 997.02 \quad 1061.44 \quad 1359.42 \quad 1385.01 \quad 1476.03$ 1485.721492 .101500 .543042 .383044 .843122 .01 3128.113148 .033148 .89

\begin{tabular}{|c|c|c|c|c|}
\hline & & $\beta_{\mathrm{ax}}$ & & \\
\hline$========$ & $======$ & $:====-$ & $======$ & $===$ \\
\hline 14.80 & 17.62 & 19.46 & 21.85 & 23.16 \\
\hline 29.59 & 31.90 & 32.58 & 36.01 & 37.86 \\
\hline 40.44 & 41.75 & 43.43 & 45.08 & 45.95 \\
\hline 51.00 & 55.94 & 56.76 & 60.76 & 63.60 \\
\hline $6.47 \quad 67.40$ & 69.26 & 72.57 & 74.49 & 77.98 \\
\hline 86.33 & 88.08 & 90.79 & 93.78 & 95.25 \\
\hline
\end{tabular}


$\begin{array}{llllll}95.79 & 99.03 & 102.05 & 106.94 & 115.79 & 123.12\end{array}$

$\begin{array}{llllll}131.00 & 132.19 & 133.73 & 143.37 & 145.44 & 163.27\end{array}$

$\begin{array}{llllll}173.93 & 177.02 & 182.69 & 188.87 & 192.16 & 196.80\end{array}$ $\begin{array}{llllll}199.77 & 200.75 & 203.01 & 207.53 & 208.56 & 212.97\end{array}$ $\begin{array}{llllll}216.86 & 221.85 & 228.67 & 233.75 & 234.86 & 244.21\end{array}$ $\begin{array}{llllll}245.76 & 246.78 & 256.26 & 259.38 & 266.60 & 280.93\end{array}$ $\begin{array}{llllll}289.87 & 293.87 & 305.30 & 309.35 & 311.13 & 312.61\end{array}$ $\begin{array}{llllll}331.82 & 336.53 & 338.51 & 343.35 & 352.80 & 358.25\end{array}$ $\begin{array}{llllll}366.23 & 370.96 & 393.70 & 395.48 & 398.52 & 404.54\end{array}$ $\begin{array}{llllll}414.66 & 416.04 & 421.34 & 422.77 & 424.31 & 426.91\end{array}$ $\begin{array}{llllll}429.61 & 455.19 & 457.73 & 462.65 & 464.06 & 481.68\end{array}$ $\begin{array}{llllll}482.49 & 485.77 & 487.16 & 490.53 & 493.43 & 499.52\end{array}$ $\begin{array}{llllll}501.32 & 502.30 & 509.06 & 527.23 & 553.64 & 553.80\end{array}$ $\begin{array}{llllll}558.94 & 561.66 & 566.51 & 601.72 & 612.26 & 613.17\end{array}$ $\begin{array}{llllll}616.32 & 616.71 & 619.12 & 621.50 & 624.40 & 625.51\end{array}$

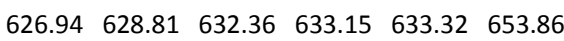
$\begin{array}{llllll}655.71 & 662.85 & 683.77 & 688.93 & 694.24 & 705.06\end{array}$ $\begin{array}{llllll}711.70 & 713.16 & 716.66 & 723.48 & 724.62 & 731.80\end{array}$ $\begin{array}{llllll}746.37 & 756.98 & 758.55 & 759.32 & 761.36 & 761.64\end{array}$ $\begin{array}{llllll}763.46 & 771.56 & 773.55 & 777.86 & 791.95 & 820.46\end{array}$ $\begin{array}{llllll}822.42 & 829.75 & 830.70 & 857.44 & 860.78 & 863.42\end{array}$ $\begin{array}{llllll}876.31 & 877.02 & 879.37 & 880.59 & 885.80 & 890.25\end{array}$ $\begin{array}{llllll}913.50 & 914.58 & 916.53 & 926.18 & 928.25 & 932.15\end{array}$

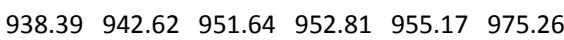

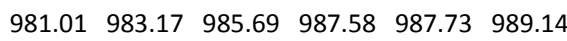

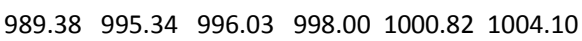
$1004.731005 .271006 .24 \quad 1007.301008 .801009 .07$ $1010.32 \quad 1011.241011 .601012 .691013 .73 \quad 1017.12$

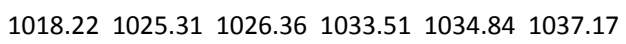

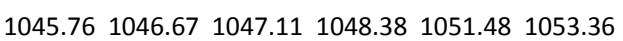
$1061.501068 .501079 .77 \quad 1080.761086 .241092 .15$

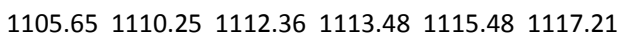

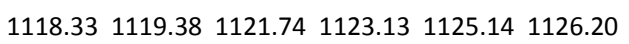

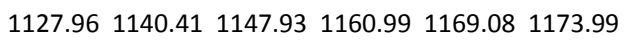
1194.161194 .191194 .601195 .631199 .221199 .64 1202.951212 .501213 .391215 .201216 .901218 .94 $1220.07 \quad 1220.911225 .58 \quad 1225.901226 .63 \quad 1227.53$

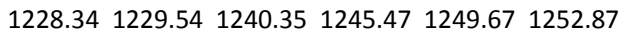
$1256.91 \quad 1263.31 \quad 1267.541273 .07 \quad 1279.991282 .51$ $1289.381302 .641306 .21 \quad 1311.98 \quad 1317.511328 .49$

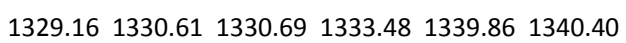

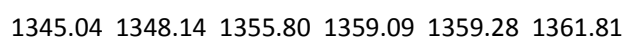

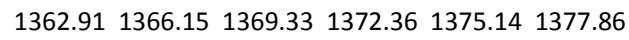

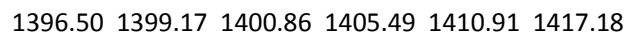

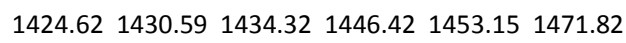

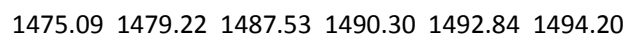

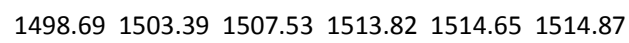

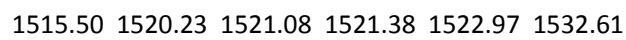
$\begin{array}{llllll}1532.90 & 1533.81 & 1537.15 & 1538.53 & 1613.56 & 1618.07\end{array}$

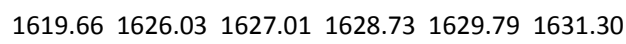
$\begin{array}{llllll}1633.58 & 1634.79 & 1640.68 & 1652.63 & 1654.76 & 1655.71\end{array}$ $2946.673004 .43 \quad 3008.673014 .873022 .723026 .12$ 3030.913044 .763053 .583054 .563063 .423075 .56 $3076.383085 .343085 .663097 .72 \quad 3099.003107 .43$ $3109.363113 .83 \quad 3114.433116 .773125 .663125 .88$ 3132.763135 .843145 .693146 .013147 .193151 .28 $\begin{array}{lllll}3154.09 & 3157.06 & 3158.813162 .68 & 3172.43 & 3172.95\end{array}$ $3175.48 \quad 3175.63 \quad 3180.113180 .73 \quad 3180.903181 .72$ 3181.973182 .493184 .853189 .873193 .263195 .77 3195.923202 .593203 .473205 .873206 .603211 .32 3238.923262 .323266 .22

\begin{tabular}{rllllll}
\multicolumn{5}{c}{$======================$} \\
\multicolumn{5}{c}{$9-\alpha_{\mathrm{ax}}$} \\
\multicolumn{1}{c}{$=======================$} \\
10.98 & 12.91 & 18.15 & 19.34 & 23.79 & 24.30 \\
26.12 & 30.39 & 31.28 & 33.47 & 37.74 & 39.51 \\
43.99 & 44.55 & 45.63 & 48.68 & 49.82 & 50.59 \\
51.80 & 56.83 & 57.61 & 60.02 & 64.50 & 66.53 \\
69.10 & 72.91 & 75.68 & 77.71 & 80.00 & 82.09 \\
84.63 & 86.48 & 89.16 & 92.24 & 95.54 & 98.22 \\
101.18 & 105.16 & 108.93 & 111.33 & 118.52 & 122.58 \\
126.42 & 130.44 & 134.21 & 137.02 & 143.23 & 152.51 \\
165.48 & 167.07 & 177.62 & 183.64 & 189.57 & 196.37 \\
197.84 & 201.37 & 203.29 & 208.59 & 215.83 & 216.14 \\
219.42 & 225.48 & 230.25 & 236.88 & 237.87 & 238.23 \\
240.35 & 251.85 & 253.95 & 259.14 & 269.83 & 276.57 \\
285.87 & 298.58 & 305.30 & 310.28 & 311.78 & 331.29 \\
331.72 & 336.33 & 337.22 & 341.01 & 355.18 & 365.35 \\
368.29 & 387.12 & 398.27 & 407.29 & 408.52 & 410.99 \\
413.42 & 413.70 & 414.56 & 417.87 & 419.11 & 420.45 \\
428.00 & 455.86 & 458.34 & 459.31 & 461.50 & 470.67 \\
478.45 & 488.17 & 491.50 & 493.89 & 496.98 & 499.07
\end{tabular}


$\begin{array}{llllll}504.41 & 518.48 & 546.97 & 554.22 & 557.71 & 558.37\end{array}$ $\begin{array}{llllll}560.56 & 571.37 & 587.74 & 609.94 & 611.96 & 616.06\end{array}$

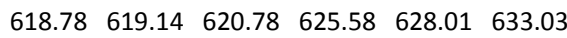
$\begin{array}{llllll}634.23 & 635.48 & 635.75 & 644.27 & 659.60 & 660.72\end{array}$ $\begin{array}{llllll}666.21 & 671.85 & 686.34 & 691.17 & 692.78 & 706.99\end{array}$ $\begin{array}{llllll}707.31 & 709.49 & 714.31 & 722.44 & 722.82 & 743.44\end{array}$ $\begin{array}{llllll}746.17 & 746.96 & 748.41 & 750.09 & 756.41 & 757.79\end{array}$ $\begin{array}{llllll}758.41 & 761.52 & 770.92 & 777.42 & 822.50 & 829.61\end{array}$ $\begin{array}{llllll}832.97 & 837.50 & 843.91 & 845.85 & 858.76 & 862.38\end{array}$ $\begin{array}{llllll}863.77 & 867.60 & 869.80 & 875.57 & 879.62 & 909.04\end{array}$

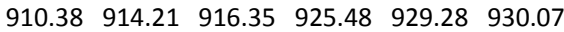
$\begin{array}{llllll}932.05 & 937.83 & 941.68 & 953.83 & 959.37 & 965.58\end{array}$

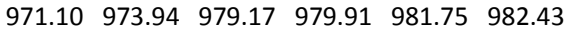
$985.45990 .72992 .11993 .87999 .06 \quad 1000.69$ $1001.251005 .631006 .07 \quad 1006.501008 .02 \quad 1014.05$

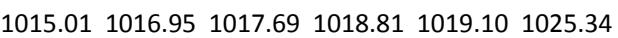

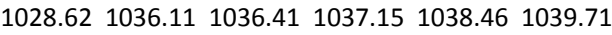

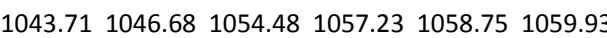
1064.021074 .911077 .711079 .951085 .411099 .06

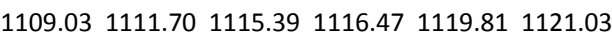

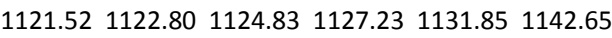

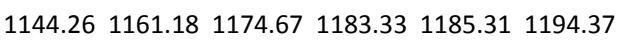
1196.071196 .401197 .561199 .291199 .671200 .09 1201.841213 .191218 .451218 .721220 .921223 .02

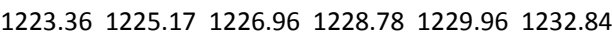
$1233.37 \quad 1237.83 \quad 1241.92 \quad 1249.43 \quad 1254.651256 .63$ 1260.891272 .041283 .691287 .011289 .051293 .76

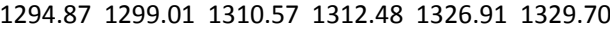
$1338.541338 .91 \quad 1340.201343 .601344 .511356 .49$

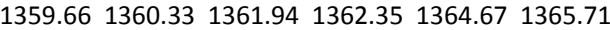

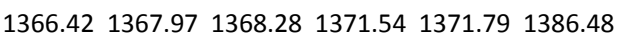

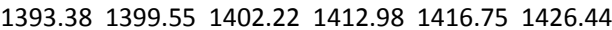
$\begin{array}{llllll}1431.36 & 1437.48 & 1447.05 & 1460.30 & 1471.82 & 1478.29\end{array}$ 1478.811493 .451494 .671496 .571497 .571498 .07

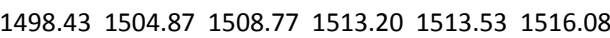

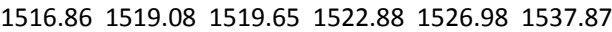
$\begin{array}{llllll}1541.35 & 1541.93 & 1545.91 & 1617.76 & 1621.87 & 1627.04\end{array}$ $1631.001633 .23 \quad 1635.691640 .661643 .67 \quad 1644.89$ 1646.331657 .871664 .751665 .071668 .142962 .06 $2986.282996 .76 \quad 3003.123032 .45 \quad 3037.02 \quad 3038.04$ 3039.123052 .113052 .513064 .993069 .533076 .33

$3081.13 \quad 3085.603091 .153107 .643109 .763110 .17$ 3113.773118 .943148 .703154 .043162 .543162 .71 3165.503167 .413176 .133176 .723177 .603179 .27 3180.863182 .783183 .903185 .253187 .173188 .55 3190.483193 .053193 .273193 .393196 .953200 .11 3201.343202 .863205 .203207 .523208 .983211 .74 3213.543214 .993217 .383224 .383232 .873250 .58 3256.283265 .59

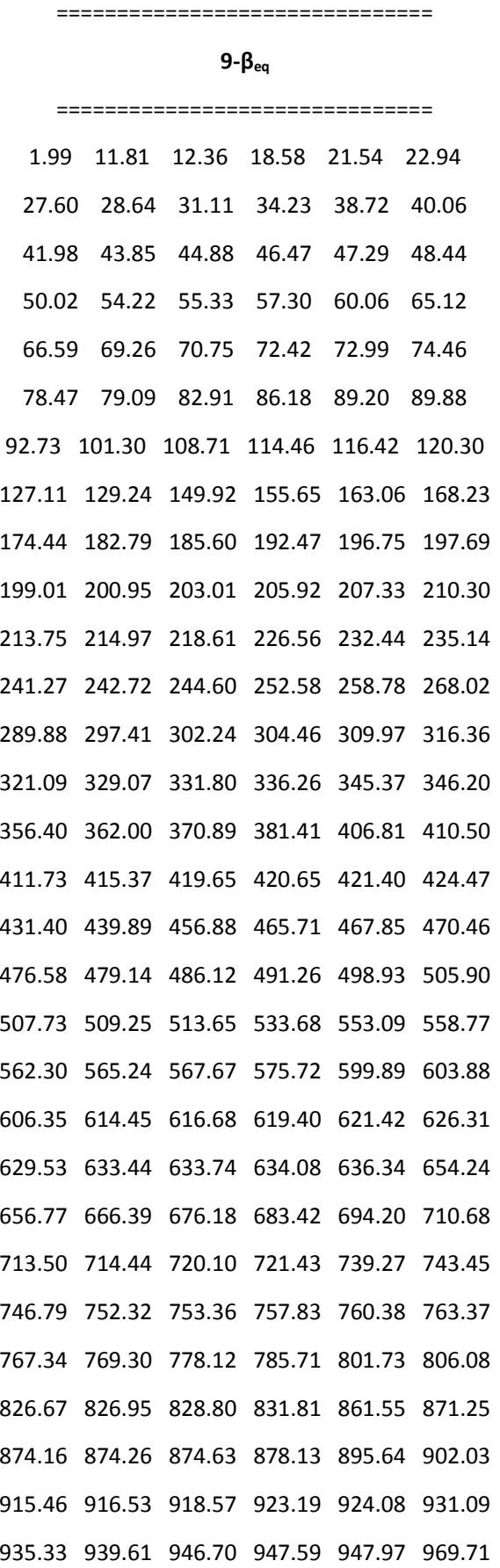




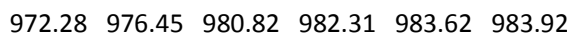

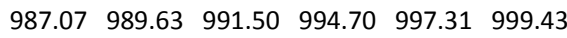
1005.24 1005.74 1006.87 1007.181008 .091009 .24 $1009.491013 .101013 .72 \quad 1014.451017 .381018 .14$ 1019.131020 .461028 .801032 .251034 .741039 .66 1044.611045 .321048 .451054 .791056 .481057 .51 1063.261068 .141071 .591085 .821090 .791095 .95 1099.981103 .401111 .961113 .521115 .461116 .03 1116.681119 .451121 .541121 .811126 .461127 .60

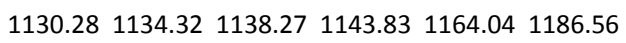
1194.301195 .241195 .421196 .431197 .991198 .82 1200.361208 .171211 .401214 .761215 .761216 .19

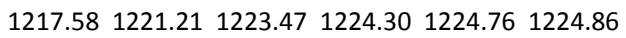

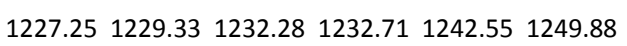

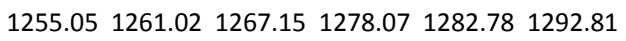

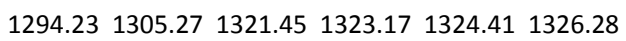
$1327.451329 .461330 .53 \quad 1333.911340 .431343 .90$

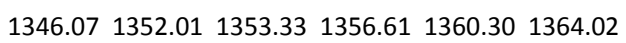
1365.171367 .071369 .351369 .821372 .001386 .92

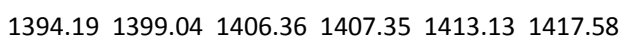
1420.381430 .751435 .441441 .571445 .591472 .58 1473.291475 .051488 .401492 .471493 .901494 .57 1495.641503 .041505 .791510 .501513 .621514 .33

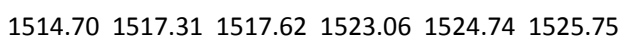
$1531.591538 .101541 .641542 .07 \quad 1612.091616 .72$ $1618.051624 .13 \quad 1624.14 \quad 1628.351631 .591637 .69$

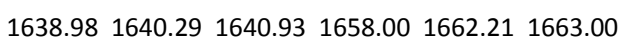
2969.033003 .743008 .373012 .343012 .693015 .10 3026.633027 .303033 .363036 .803048 .003058 .48 3061.043064 .703069 .283080 .343083 .573085 .06 3087.053100 .003107 .863114 .843129 .983138 .17 3149.383149 .393155 .853159 .773163 .883165 .65 3166.993168 .283171 .703171 .903177 .553177 .73 $3178.403178 .673181 .003181 .823187 .23 \quad 3187.97$ 3192.623195 .903198 .433198 .543199 .973201 .55 3203.183205 .703208 .923209 .843216 .983226 .70

3227.813266 .023276 .73

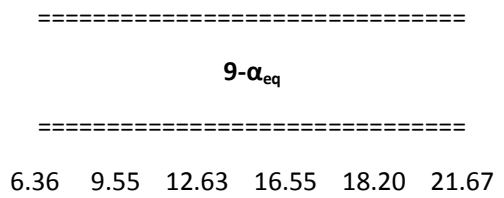

$\begin{array}{llllll}22.05 & 23.35 & 25.27 & 27.54 & 30.53 & 32.25\end{array}$ $\begin{array}{llllll}34.91 & 39.10 & 40.59 & 40.83 & 41.84 & 44.20\end{array}$ $\begin{array}{llllll}46.77 & 49.13 & 51.10 & 52.14 & 53.71 & 57.30\end{array}$ $\begin{array}{llllll}59.84 & 61.70 & 63.35 & 67.41 & 69.60 & 71.08\end{array}$ $\begin{array}{llllll}73.08 & 75.90 & 81.66 & 83.22 & 87.98 & 91.77\end{array}$ $\begin{array}{llllll}95.85 & 100.69 & 107.08 & 111.86 & 116.24 & 117.70\end{array}$ $\begin{array}{llllll}121.86 & 125.17 & 125.86 & 134.38 & 147.26 & 156.53\end{array}$ $\begin{array}{llllll}165.20 & 166.97 & 176.88 & 182.54 & 183.78 & 190.71\end{array}$ $\begin{array}{llllll}193.70 & 196.54 & 200.45 & 204.40 & 215.18 & 216.50\end{array}$ $\begin{array}{llllll}222.55 & 224.85 & 231.65 & 234.75 & 242.36 & 243.54\end{array}$ $\begin{array}{llllll}246.08 & 248.43 & 255.84 & 258.54 & 265.35 & 273.53\end{array}$ $\begin{array}{llllll}288.48 & 296.83 & 302.85 & 303.12 & 310.38 & 320.05\end{array}$

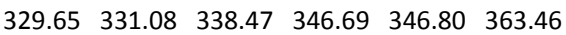
$\begin{array}{llllll}371.45 & 383.07 & 389.77 & 402.96 & 403.83 & 409.64\end{array}$ $\begin{array}{llllll}415.79 & 417.74 & 418.88 & 419.49 & 422.26 & 422.59\end{array}$ $\begin{array}{llllll}430.55 & 439.03 & 450.10 & 456.13 & 461.51 & 462.81\end{array}$ $\begin{array}{llllll}472.31 & 475.91 & 482.69 & 497.12 & 499.08 & 504.35\end{array}$ $\begin{array}{llllll}510.67 & 511.76 & 522.30 & 530.39 & 552.05 & 558.40\end{array}$ $\begin{array}{llllll}567.44 & 569.96 & 588.21 & 599.54 & 606.24 & 608.99\end{array}$ $\begin{array}{llllll}610.09 & 618.68 & 619.84 & 621.95 & 624.69 & 627.28\end{array}$ $\begin{array}{llllll}631.11 & 632.96 & 634.73 & 635.50 & 647.35 & 660.03\end{array}$ $\begin{array}{llllll}660.80 & 661.13 & 669.41 & 690.44 & 692.78 & 699.88\end{array}$

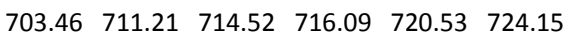
$\begin{array}{llllll}745.40 & 747.01 & 750.84 & 751.38 & 752.15 & 758.50\end{array}$

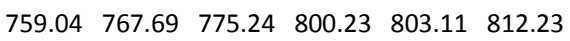
$\begin{array}{llllll}815.83 & 826.30 & 827.11 & 848.64 & 851.36 & 857.97\end{array}$ $\begin{array}{llllll}858.21 & 863.78 & 866.65 & 869.13 & 870.04 & 871.17\end{array}$

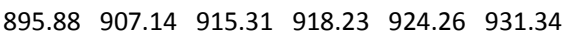

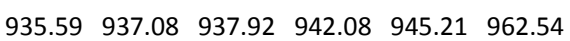

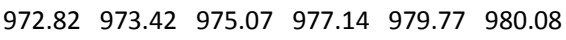

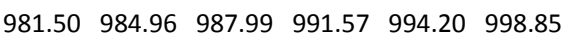
$1002.351002 .781003 .08 \quad 1006.051006 .591007 .94$ $1008.781009 .571009 .93 \quad 1017.08 \quad 1017.191017 .59$ $1018.311021 .101036 .03 \quad 1037.02 \quad 1037.861038 .28$ 1039.961048 .051053 .011056 .751058 .051059 .53

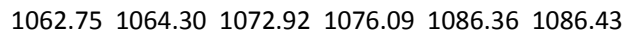

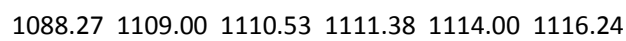

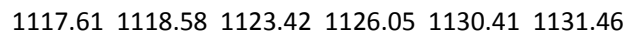

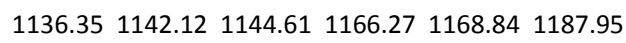
1192.321196 .221196 .581196 .771198 .511199 .85 1200.011205 .511210 .071211 .691216 .991218 .38 


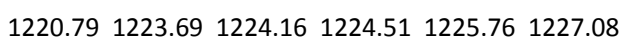

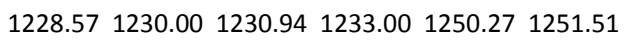

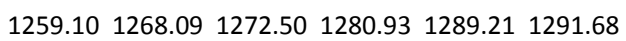
1296.301306 .131313 .411319 .661320 .601326 .63

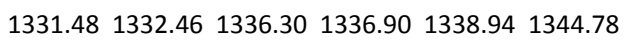
1355.371356 .161358 .171360 .921361 .911362 .53 $1366.551367 .02 \quad 1367.601368 .851370 .641374 .98$ $\begin{array}{llllll}1383.84 & 1387.70 & 1392.18 & 1397.27 & 1406.11 & 1407.76\end{array}$ $1415.251435 .891436 .91 \quad 1447.82 \quad 1464.881474 .59$

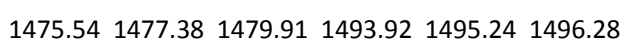

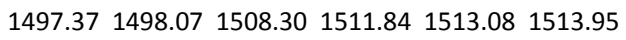

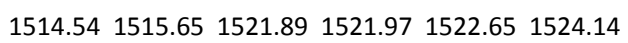
1537.391540 .601541 .421541 .911614 .841620 .86 $1628.761629 .841630 .841632 .53 \quad 1640.82 \quad 1641.20$ 1641.981642 .641660 .611661 .561663 .491664 .55 2983.003004 .773029 .403034 .443038 .073040 .00 3042.513044 .103045 .553048 .053054 .263054 .79 3063.723076 .973082 .763092 .353093 .113098 .22 3114.973118 .783119 .003145 .373158 .223161 .86 3164.273166 .913174 .903175 .443178 .413180 .24 3180.633181 .853185 .463186 .003186 .063188 .92 3189.703190 .413195 .293198 .343198 .493199 .14 3199.363199 .903204 .053205 .633206 .763208 .21 3209.033209 .043210 .533229 .663238 .193240 .28 3254.193271 .493291 .42

\begin{tabular}{cccccc}
\multicolumn{5}{c}{$=$ 4- $\beta$} \\
\multicolumn{1}{c}{$======================$} \\
11.07 & 15.85 & 22.13 & 24.28 & 28.98 & 35.52 \\
40.12 & 42.20 & 52.28 & 54.66 & 64.00 & 69.36 \\
74.48 & 75.84 & 92.05 & 96.74 & 98.12 & 107.68 \\
125.22 & 133.15 & 146.25 & 147.88 & 157.15 & 172.47 \\
186.01 & 199.96 & 204.32 & 228.20 & 238.25 & 239.61 \\
251.59 & 257.18 & 261.21 & 265.18 & 276.63 & 307.71 \\
311.84 & 324.29 & 330.77 & 332.00 & 355.63 & 372.31 \\
383.57 & 392.46 & 403.45 & 415.03 & 416.45 & 420.43 \\
424.50 & 427.81 & 448.48 & 478.11 & 483.84 & 489.41 \\
491.21 & 499.42 & 547.71 & 568.19 & 584.25 & 602.98 \\
610.89 & 622.77 & 631.99 & 632.92 & 633.58 & 634.12 \\
636.10 & 677.94 & 709.70 & 711.61 & 714.63 & 715.62 \\
719.08 & 736.97 & 755.88 & 757.64 & 760.28 & 762.26
\end{tabular}

$\begin{array}{llllll}764.85 & 796.57 & 823.70 & 825.83 & 827.90 & 830.42\end{array}$ $\begin{array}{llllll}861.85 & 862.61 & 866.12 & 870.22 & 876.56 & 897.97\end{array}$ $\begin{array}{llllll}914.64 & 924.01 & 925.84 & 926.99 & 932.20 & 939.41\end{array}$ $\begin{array}{llllll}960.81 & 977.03 & 979.38 & 981.67 & 985.43 & 987.39\end{array}$

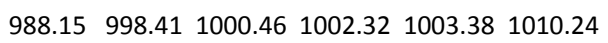

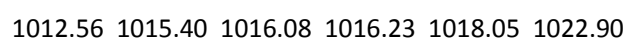
$\begin{array}{llllll}1028.33 & 1039.71 & 1042.02 & 1053.46 & 1055.59 & 1055.72\end{array}$

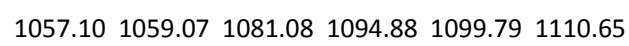

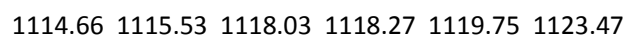
$\begin{array}{llllll}1128.57 & 1133.54 & 1145.28 & 1178.21 & 1182.49 & 1193.32\end{array}$ 1193.951194 .071195 .841212 .651213 .451214 .12 $\begin{array}{llllll}1215.19 & 1226.32 & 1226.74 & 1227.40 & 1236.18 & 1241.28\end{array}$ $\begin{array}{llllll}1246.71 & 1261.70 & 1268.91 & 1275.34 & 1282.87 & 1286.94\end{array}$ $\begin{array}{llllll}1294.46 & 1303.31 & 1309.35 & 1319.06 & 1340.69 & 1344.44\end{array}$ $\begin{array}{llllll}1350.31 & 1353.79 & 1355.63 & 1360.13 & 1362.79 & 1363.54\end{array}$ $\begin{array}{llllll}1363.87 & 1365.51 & 1368.76 & 1379.72 & 1387.96 & 1394.05\end{array}$ $\begin{array}{llllll}1399.73 & 1401.32 & 1409.71 & 1416.03 & 1430.43 & 1437.21\end{array}$ $\begin{array}{lllllll}1441.89 & 1448.38 & 1487.44 & 1493.08 & 1494.62 & 1495.49\end{array}$ $\begin{array}{llllll}1496.78 & 1500.99 & 1506.37 & 1508.89 & 1513.17 & 1516.70\end{array}$ $\begin{array}{llllll}1519.56 & 1524.73 & 1538.16 & 1538.33 & 1538.97 & 1540.88\end{array}$ $\begin{array}{llllll}1541.92 & 1638.24 & 1640.32 & 1642.58 & 1642.63 & 1660.29\end{array}$ $1661.611662 .811665 .522968 .52 \quad 2987.072999 .50$ 3000.353008 .623011 .483030 .093032 .053035 .83 $3046.313055 .773060 .173066 .53 \quad 3069.953074 .33$

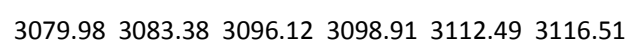
$3151.603161 .113165 .393167 .63 \quad 3168.28 \quad 3171.24$

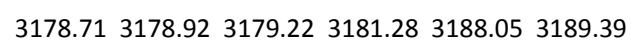
3190.713192 .803198 .083198 .513199 .643209 .85 3209.993210 .103223 .30

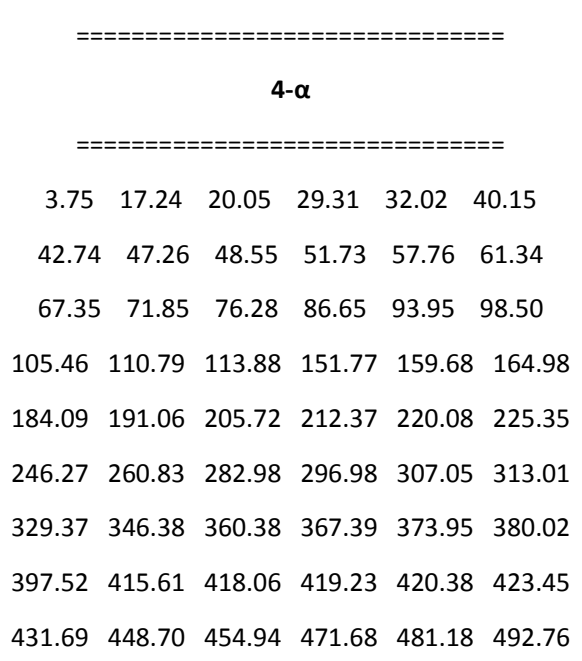


$\begin{array}{llllll}497.32 & 504.37 & 519.26 & 578.62 & 600.86 & 615.54\end{array}$ $\begin{array}{llllll}618.43 & 627.95 & 631.30 & 632.29 & 633.35 & 634.03\end{array}$ $\begin{array}{llllll}634.79 & 658.22 & 676.17 & 708.56 & 713.74 & 714.77\end{array}$ $\begin{array}{llllll}716.96 & 721.34 & 752.35 & 758.14 & 761.40 & 768.78\end{array}$ $\begin{array}{llllll}780.05 & 797.04 & 820.81 & 824.02 & 825.45 & 833.21\end{array}$ $\begin{array}{llllll}853.96 & 859.32 & 863.01 & 871.08 & 872.01 & 872.72\end{array}$

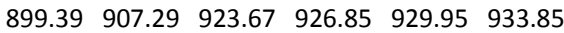
$\begin{array}{llllll}943.88 & 977.60 & 978.67 & 984.57 & 984.78 & 987.97\end{array}$ 989.31998 .411000 .761002 .821005 .011008 .09 1011.551014 .531015 .911016 .571018 .281020 .02 $1030.271048 .921053 .33 \quad 1054.751055 .911056 .82$

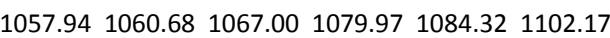
$1107.691112 .96 \quad 1116.21 \quad 1119.58 \quad 1120.151121 .91$ $1122.871133 .901147 .401160 .67 \quad 1174.211191 .81$ 1194.811195 .311198 .081211 .501212 .011214 .20 $1214.671228 .141230 .04 \quad 1231.791232 .03 \quad 1246.09$

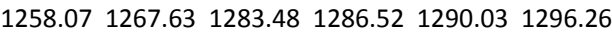

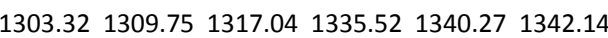
$1357.21 \quad 1361.491362 .22 \quad 1363.381364 .43 \quad 1365.08$ $1365.301368 .48 \quad 1373.351385 .25 \quad 1398.201403 .86$ 1406.831411 .991413 .051427 .301430 .081431 .68 1441.701448 .111477 .571494 .051495 .241496 .47 $1497.091505 .43 \quad 1508.221509 .491511 .291513 .29$ $1514.191517 .61 \quad 1524.97 \quad 1537.64 \quad 1538.46 \quad 1538.56$ 1540.411640 .821642 .091642 .761642 .901660 .83 1661.551662 .361662 .953002 .093024 .173026 .74 3027.703029 .863041 .003048 .653053 .263054 .66 3071.703074 .323075 .473076 .993086 .773091 .72 $3099.163103 .493104 .263106 .33 \quad 3110.273115 .40$ 3121.463160 .063165 .903167 .923168 .163171 .48 3177.303177 .823178 .003181 .243185 .903186 .83 3188.933191 .863195 .773198 .333200 .593201 .06 3204.643222 .373243 .96

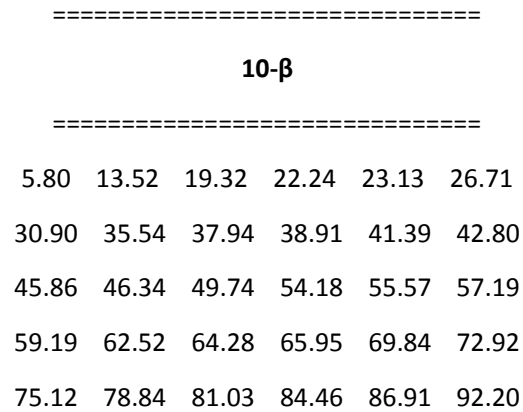

$\begin{array}{llllll}94.16 & 97.32 & 106.82 & 109.25 & 114.38 & 115.75\end{array}$ $\begin{array}{llllll}130.86 & 145.78 & 151.83 & 159.33 & 160.61 & 162.60\end{array}$ $\begin{array}{llllll}172.37 & 174.50 & 178.77 & 187.91 & 188.84 & 192.31\end{array}$ $199.99206 .42 \quad 213.69217 .19 \quad 219.15 \quad 228.41$ $\begin{array}{llllll}232.66 & 236.26 & 244.41 & 247.88 & 254.13 & 257.08\end{array}$ $\begin{array}{llllll}259.44 & 264.93 & 275.65 & 282.62 & 284.77 & 293.29\end{array}$ $\begin{array}{llllll}308.73 & 313.37 & 320.47 & 331.10 & 340.74 & 344.57\end{array}$ $\begin{array}{llllll}353.61 & 360.98 & 364.90 & 378.02 & 394.80 & 405.25\end{array}$ $\begin{array}{llllll}411.75 & 411.92 & 415.18 & 415.41 & 416.77 & 417.66\end{array}$ $\begin{array}{llllll}421.92 & 425.86 & 432.95 & 451.12 & 456.34 & 460.62\end{array}$ $\begin{array}{llllll}468.59 & 482.88 & 483.74 & 494.24 & 499.63 & 506.84\end{array}$ $\begin{array}{llllll}520.52 & 527.71 & 557.87 & 571.47 & 574.50 & 597.81\end{array}$ $\begin{array}{llllll}608.31 & 614.70 & 616.34 & 616.41 & 619.91 & 622.72\end{array}$ $\begin{array}{llllll}625.07 & 632.17 & 632.78 & 635.49 & 635.93 & 638.65\end{array}$ $\begin{array}{llllll}653.21 & 658.27 & 664.58 & 682.71 & 694.22 & 698.99\end{array}$

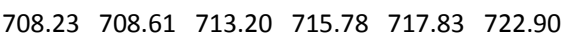
$\begin{array}{llllll}745.11 & 750.62 & 752.78 & 754.24 & 755.94 & 759.10\end{array}$ $\begin{array}{llllll}763.89 & 773.61 & 778.44 & 778.91 & 786.86 & 820.69\end{array}$ $\begin{array}{llllll}828.92 & 837.03 & 843.52 & 846.73 & 858.44 & 860.09\end{array}$ $\begin{array}{lllll}862.63 \quad 865.86 & 868.89 & 873.98 & 875.01 & 891.08\end{array}$

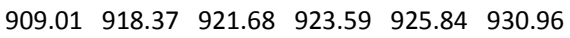

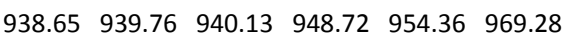

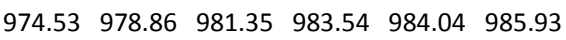
$987.95989 .86996 .96 \quad 1001.78 \quad 1002.32 \quad 1003.07$ 1005.181006 .591007 .261008 .741011 .051013 .78 1013.991015 .661017 .081018 .681021 .191021 .65 $1025.421026 .73 \quad 1030.351032 .501033 .731045 .33$ 1046.411050 .031056 .311056 .551057 .561060 .28 $1071.871075 .67 \quad 1076.13 \quad 1082.82 \quad 1085.161095 .30$ 1100.601101 .461111 .201113 .341115 .631117 .18 1117.841119 .531120 .691125 .011125 .471128 .28 1132.611141 .571176 .981181 .701196 .261196 .86 1197.501199 .031203 .001204 .051204 .801207 .08 1213.871214 .331216 .771220 .211226 .061226 .90 1227.101228 .201232 .381236 .091240 .541244 .85 $1253.53 \quad 1264.04 \quad 1266.18 \quad 1269.48 \quad 1271.99 \quad 1272.75$

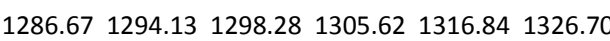
$1331.131334 .62 \quad 1337.11 \quad 1338.101339 .861342 .88$ $1345.051359 .53 \quad 1361.451362 .551363 .03 \quad 1363.28$

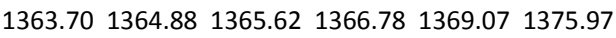
1384.461395 .091399 .031404 .071405 .811417 .58 


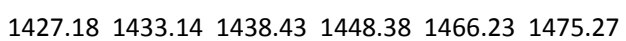
$\begin{array}{llllll}1477.66 & 1477.89 & 1490.93 & 1495.26 & 1495.82 & 1497.41\end{array}$ $1499.491504 .851508 .48 \quad 1511.491514 .191514 .86$ 1515.541519 .351519 .711520 .631526 .291535 .03 1539.361539 .741541 .461544 .901609 .421618 .93 $1627.27 \quad 1628.591630 .38 \quad 1631.901638 .74 \quad 1639.35$

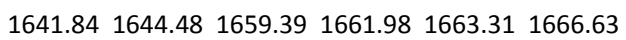
$2964.32 \quad 2977.632990 .382996 .62 \quad 3017.953034 .74$ 3037.793040 .533041 .203042 .643053 .583056 .95 3070.193075 .793094 .913102 .423110 .543123 .27 $3128.683129 .33 \quad 3140.663166 .003168 .783172 .89$ 3175.603178 .823179 .393180 .693181 .243182 .36 3184.023185 .283190 .163190 .703191 .863193 .62 3193.643193 .693197 .783198 .583201 .493201 .58 3203.483204 .113204 .623208 .833208 .903209 .97 3214.753214 .923216 .313218 .533229 .313229 .81 3240.983243 .073251 .42

\begin{tabular}{rllllll}
\multicolumn{1}{c}{$=======================$} \\
\multicolumn{5}{c}{$10-\alpha$} \\
3.29 & 11.78 & 13.94 & 15.24 & 21.03 & 26.59 \\
27.60 & 29.35 & 29.96 & 35.51 & 39.15 & 40.36 \\
41.43 & 42.38 & 45.02 & 45.62 & 49.46 & 50.33 \\
52.66 & 54.67 & 55.73 & 57.77 & 62.01 & 64.67 \\
67.02 & 70.71 & 74.37 & 76.12 & 80.29 & 84.94 \\
89.14 & 95.97 & 100.07 & 101.34 & 106.46 & 111.18 \\
117.68 & 120.58 & 132.24 & 137.97 & 150.99 & 154.96 \\
165.01 & 173.15 & 180.20 & 187.08 & 191.10 & 194.01 \\
196.99 & 206.41 & 208.63 & 213.14 & 216.06 & 218.60 \\
223.21 & 229.38 & 232.78 & 242.48 & 245.32 & 248.29 \\
251.81 & 261.30 & 266.94 & 277.11 & 296.83 & 309.13 \\
314.59 & 326.29 & 331.08 & 336.35 & 354.53 & 359.68 \\
366.36 & 370.47 & 383.51 & 391.69 & 401.50 & 405.34 \\
414.05 & 414.59 & 415.80 & 418.03 & 418.08 & 419.13 \\
419.87 & 426.49 & 439.47 & 451.00 & 457.07 & 458.19 \\
471.81 & 494.20 & 495.56 & 498.08 & 504.28 & 508.14 \\
515.96 & 519.45 & 556.72 & 572.09 & 574.72 & 585.35 \\
602.48 & 612.81 & 617.81 & 618.61 & 619.37 & 623.79 \\
630.00 & 633.01 & 634.21 & 634.90 & 634.92 & 639.26 \\
643.89 & 656.97 & 657.45 & 668.08 & 688.37 & 688.97 \\
689.67 & 704.21 & 710.89 & 714.13 & 720.86 & 721.85
\end{tabular}

$\begin{array}{llllll}725.09 & 750.04 & 753.33 & 758.82 & 759.76 & 762.35\end{array}$ $\begin{array}{llllll}763.24 & 774.25 & 776.36 & 799.92 & 808.83 & 812.08\end{array}$ $\begin{array}{llllll}830.65 & 833.55 & 834.67 & 841.89 & 848.35 & 856.14\end{array}$ $\begin{array}{llllll}859.88 & 863.33 & 867.74 & 871.50 & 874.24 & 875.89\end{array}$ $\begin{array}{llllll}888.96 & 897.74 & 912.06 & 927.71 & 934.36 & 935.01\end{array}$ $\begin{array}{llllll}937.89 & 938.78 & 944.82 & 953.21 & 957.88 & 961.41\end{array}$ $\begin{array}{llllll}972.82 & 978.81 & 981.79 & 984.39 & 986.11 & 987.31\end{array}$ $988.06990 .69994 .55995 .54 \quad 1000.841003 .21$ $1006.741007 .14 \quad 1007.751010 .851011 .381012 .86$ $\begin{array}{llll}1013.96 & 1016.961018 .21 & 1018.631018 .94 & 1021.04\end{array}$ $\begin{array}{llllll}1022.05 & 1023.84 & 1031.48 & 1032.52 & 1033.10 & 1038.09\end{array}$ $\begin{array}{llllll}1048.72 & 1051.73 & 1054.99 & 1056.79 & 1057.30 & 1057.67\end{array}$

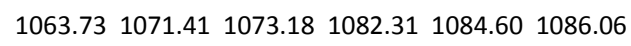
$\begin{array}{llllll}1101.24 & 1110.03 & 1111.07 & 1111.98 & 1115.30 & 1117.50\end{array}$

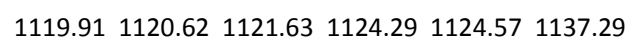
$\begin{array}{llllll}1142.89 & 1170.47 & 1174.10 & 1178.87 & 1196.65 & 1197.99\end{array}$ $\begin{array}{lllll}1198.30 & 1199.26 & 1203.02 & 1203.631204 .70 & 1211.60\end{array}$ $\begin{array}{llllll}1214.74 & 1216.05 & 1219.22 & 1222.53 & 1223.43 & 1226.93\end{array}$ $\begin{array}{llllll}1227.88 & 1230.40 & 1231.15 & 1235.17 & 1236.58 & 1243.14\end{array}$ $\begin{array}{lllllll}1248.85 & 1259.28 & 1267.28 & 1273.71 & 1275.85 & 1282.38\end{array}$ $\begin{array}{llllll}1286.11 & 1289.22 & 1300.17 & 1307.60 & 1319.28 & 1330.29\end{array}$ $\begin{array}{llllll}1331.85 & 1333.85 & 1335.17 & 1338.36 & 1338.95 & 1354.26\end{array}$ $\begin{array}{llllll}1357.12 & 1358.21 & 1359.23 & 1360.51 & 1361.54 & 1363.36\end{array}$ $\begin{array}{llllll}1364.27 & 1364.67 & 1365.58 & 1367.72 & 1369.62 & 1371.11\end{array}$ $\begin{array}{llllll}1387.65 & 1391.06 & 1395.68 & 1405.87 & 1408.43 & 1410.93\end{array}$ $\begin{array}{llllll}1422.15 & 1439.73 & 1441.01 & 1446.47 & 1456.59 & 1473.67\end{array}$ $\begin{array}{llllll}1476.94 & 1478.84 & 1479.95 & 1495.44 & 1496.12 & 1496.14\end{array}$ $\begin{array}{llllll}1496.73 & 1499.83 & 1501.98 & 1506.31 & 1511.98 & 1513.45\end{array}$ $\begin{array}{llllll}1514.73 & 1514.85 & 1515.30 & 1519.19 & 1520.02 & 1521.74\end{array}$ $\begin{array}{llllll}1538.83 & 1540.90 & 1541.39 & 1542.15 & 1611.36 & 1614.35\end{array}$ $\begin{array}{llllll}1624.89 & 1627.15 & 1627.98 & 1631.50 & 1639.68 & 1640.22\end{array}$ $\begin{array}{llllll}1641.20 & 1642.62 & 1660.38 & 1660.40 & 1663.14 & 1663.28\end{array}$ $2987.12 \quad 2996.853010 .013024 .78 \quad 3026.98 \quad 3028.61$ $3036.92 \quad 3045.103060 .473061 .003065 .853066 .69$

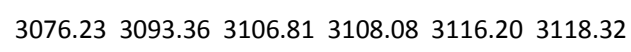

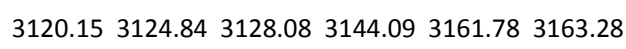

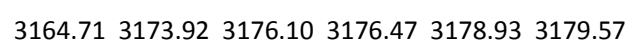

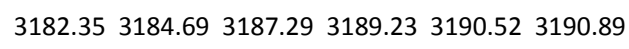
3191.213191 .273197 .023197 .733197 .773198 .05 3200.923201 .883204 .593204 .953205 .843207 .48 3207.653210 .913212 .613216 .403217 .063223 .22 


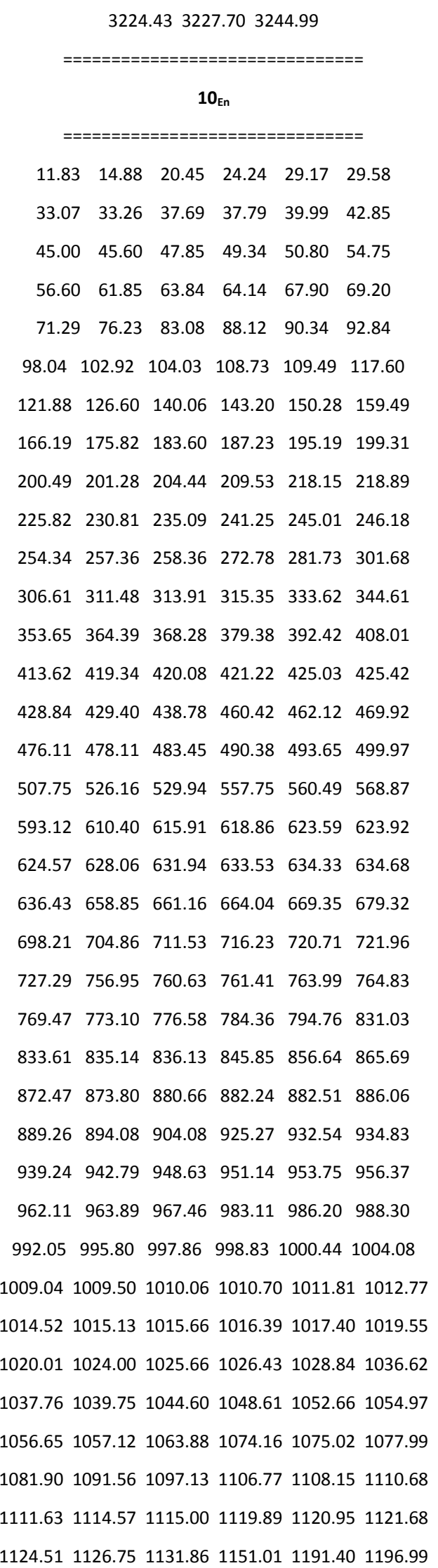

1198.941199 .121199 .221199 .491200 .511201 .18 1202.361205 .101218 .021218 .631220 .311221 .59 $1224.37 \quad 1228.13 \quad 1230.18 \quad 1231.01 \quad 1232.691233 .64$

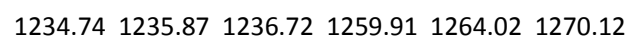
1273.841279 .401287 .101291 .401296 .761308 .95

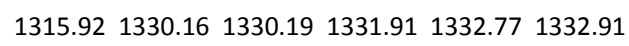
$1347.07 \quad 1351.191353 .851356 .71 \quad 1358.961360 .18$ 1361.461362 .461364 .541365 .361374 .241376 .07

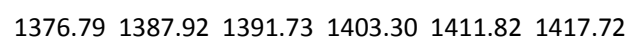
$1421.011437 .951442 .371443 .47 \quad 1447.371472 .51$ 1473.661475 .821476 .491493 .331495 .901497 .15

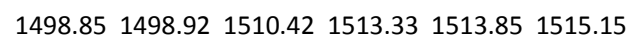

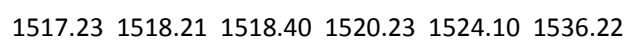
1540.141540 .561545 .881546 .861619 .921623 .44

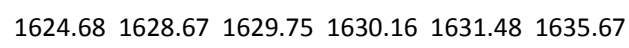
1639.401643 .131650 .231656 .981659 .241664 .31 2923.962964 .042981 .762994 .413000 .543027 .27 3027.443039 .453041 .103048 .883050 .973058 .48 3070.883071 .173085 .533105 .783111 .543117 .60 3131.093136 .193139 .683140 .913154 .923156 .72 3164.663165 .233168 .593170 .353174 .573177 .96 3179.453179 .823180 .323184 .133184 .343185 .49 3185.973186 .623188 .013188 .373189 .853191 .26 3196.173198 .563199 .713201 .243201 .703204 .16 3207.123207 .313211 .093212 .203222 .753228 .68 3235.833251 .443267 .27

\begin{tabular}{|c|c|c|c|c|c|}
\hline \multicolumn{6}{|c|}{ 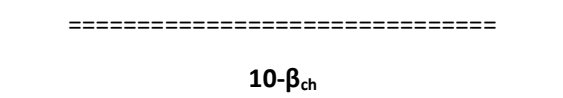 } \\
\hline \multicolumn{6}{|c|}{ 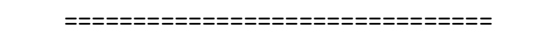 } \\
\hline 18.29 & 21.06 & 21.83 & 27.79 & 31.90 & 32.63 \\
\hline 34.30 & 37.04 & 40.56 & 42.53 & 43.57 & 45.08 \\
\hline 46.81 & 50.84 & 52.65 & 54.50 & 56.12 & 58.97 \\
\hline 61.33 & 65.85 & 69.80 & 74.18 & 76.89 & 79.17 \\
\hline 86.71 & 88.14 & 94.96 & 97.341 & 100.691 & 102.88 \\
\hline 104.19 & 109.49 & 112.37 & 116.02 & 127.12 & 128.89 \\
\hline 131.99 & 134.70 & 146.48 & 148.15 & 159.05 & 171.97 \\
\hline 174.88 & 177.96 & 186.18 & 193.00 & 195.90 & 199.75 \\
\hline 202.71 & 213.45 & 215.74 & 217.41 & L 221.44 & 223.37 \\
\hline 232.84 & 233.88 & 240.24 & 247.47 & 251.05 & 253.86 \\
\hline 259.06 & 266.27 & 270.26 & 285.78 & 301.64 & 312.35 \\
\hline 320.32 & 330.55 & 334.62 & 339.24 & 342.24 & 368.14 \\
\hline
\end{tabular}


$\begin{array}{llllll}372.34 & 386.86 & 390.35 & 401.44 & 407.01 & 410.67\end{array}$ $\begin{array}{llllll}419.39 & 420.15 & 420.73 & 426.69 & 430.03 & 430.37\end{array}$ $\begin{array}{llllll}436.17 & 446.08 & 457.07 & 458.26 & 464.07 & 466.01\end{array}$ $\begin{array}{llllll}478.05 & 483.41 & 492.01 & 496.89 & 501.78 & 507.25\end{array}$ $\begin{array}{llllll}511.93 & 518.32 & 552.87 & 569.97 & 574.33 & 591.64\end{array}$ $\begin{array}{llllll}610.04 & 614.34 & 616.94 & 618.37 & 621.74 & 623.57\end{array}$ $\begin{array}{llllll}627.48 & 630.97 & 632.43 & 633.86 & 635.50 & 642.87\end{array}$ $\begin{array}{llllll}649.62 & 657.24 & 657.93 & 666.49 & 692.81 & 696.40\end{array}$ $\begin{array}{llllll}699.02 & 702.57 & 718.32 & 721.27 & 724.31 & 729.43\end{array}$ $\begin{array}{llllll}741.64 & 751.72 & 755.11 & 761.99 & 763.26 & 768.52\end{array}$

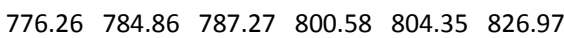
$\begin{array}{llllll}832.22 & 834.48 & 837.82 & 860.48 & 865.36 & 872.21\end{array}$ $\begin{array}{llllll}877.78 & 879.23 & 879.82 & 887.14 & 890.29 & 896.76\end{array}$

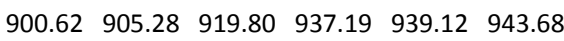

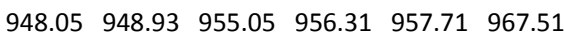

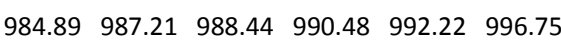
998.961000 .921001 .761005 .761007 .241007 .93 1009.231010 .511011 .561012 .181013 .781015 .09

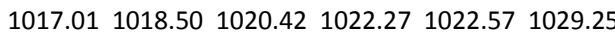

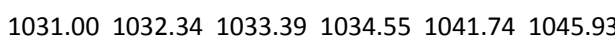
$1049.37 \quad 1050.07 \quad 1051.47 \quad 1054.73 \quad 1056.201066 .01$

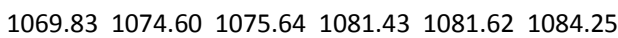

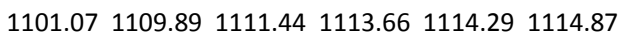
1115.401117 .831119 .821121 .601122 .341124 .14 1132.641144 .761157 .221162 .641196 .671197 .69 1199.311199 .801202 .601203 .101203 .851213 .86 1215.751216 .691219 .201219 .331222 .011223 .10

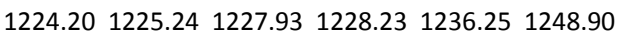

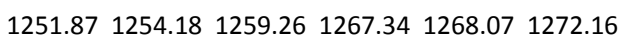

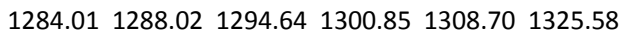

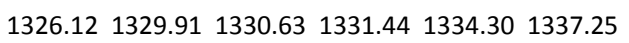

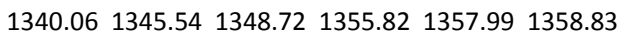

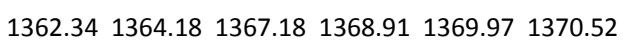
1372.821392 .001395 .591404 .061413 .941416 .52 1423.381439 .381440 .321442 .601457 .681472 .91

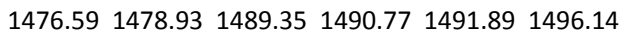

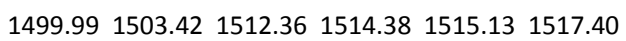

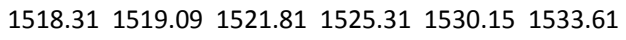
$\begin{array}{llllll}1535.73 & 1537.21 & 1539.38 & 1540.87 & 1613.30 & 1617.28\end{array}$ $\begin{array}{llllll}1621.28 & 1623.62 & 1623.66 & 1627.10 & 1629.40 & 1631.08\end{array}$ 1631.161637 .591642 .521648 .611650 .901659 .33
$2901.03 \quad 2951.452992 .232995 .893008 .573017 .05$ 3021.013023 .903034 .713043 .203046 .213056 .92 3061.823064 .873086 .973103 .613110 .203111 .76 3114.503128 .093129 .693138 .043141 .543145 .55 3145.983148 .583151 .283153 .803159 .253162 .19 3165.743167 .923170 .773173 .143176 .293178 .75 3179.463180 .623182 .413182 .563184 .893186 .05 $3190.283193 .03 \quad 3193.123194 .263198 .173202 .22$ $3202.973204 .13 \quad 3207.073208 .483211 .643213 .42$ 3213.783234 .123259 .86

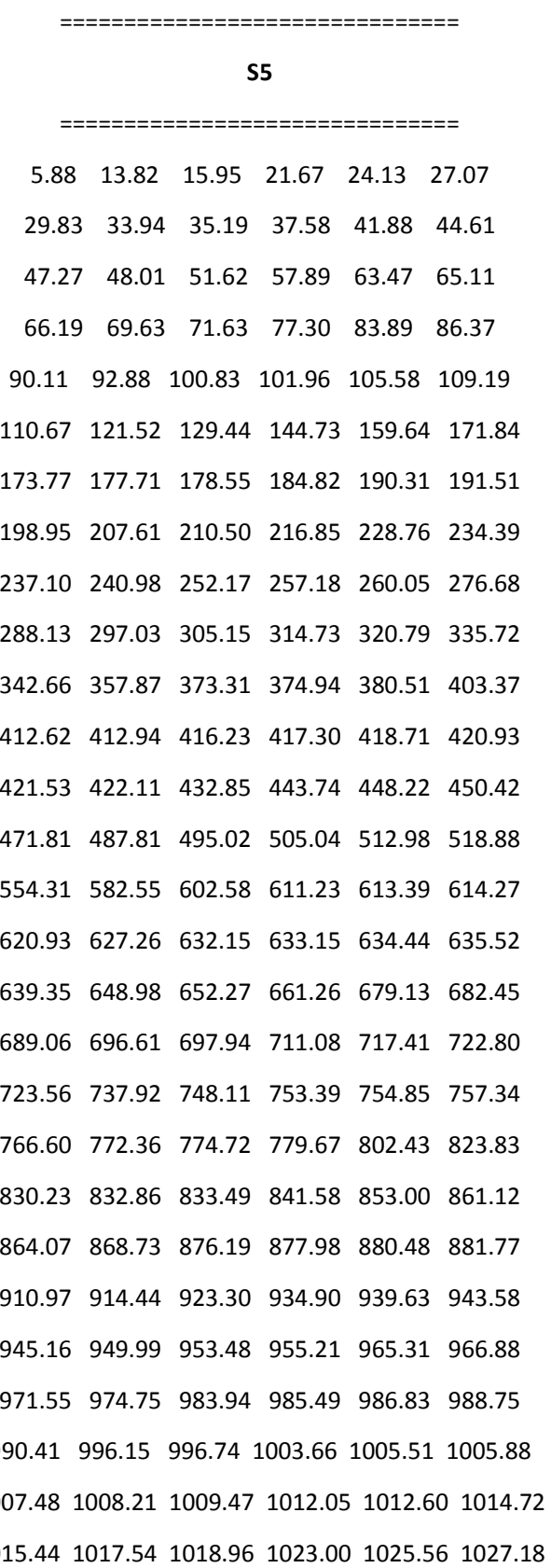


$1027.97 \quad 1028.73 \quad 1030.05 \quad 1031.04 \quad 1032.46 \quad 1033.58$

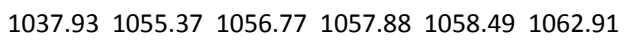

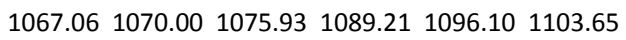
1110.641113 .931114 .581116 .501118 .651120 .04 $1120.731122 .98 \quad 1124.38 \quad 1129.06 \quad 1137.051145 .97$ 1152.701167 .841197 .901200 .441201 .001201 .12 $1204.301207 .58 \quad 1209.191216 .301217 .591218 .38$ 1219.221219 .411220 .401230 .601231 .041232 .20 $\begin{array}{llllll}1232.51 & 1238.63 & 1242.25 & 1252.94 & 1258.72 & 1267.99\end{array}$ $\begin{array}{llll}1276.01 & 1280.161292 .68 & 1297.87 & 1300.631317 .09\end{array}$ $1329.191330 .901336 .27 \quad 1337.73 \quad 1342.091345 .15$

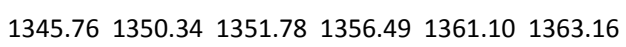
1364.711365 .651366 .961368 .211369 .251370 .07

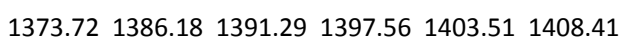

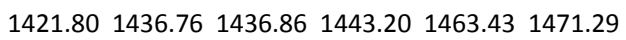
$\begin{array}{llllll}1474.60 & 1476.73 & 1483.33 & 1493.22 & 1494.03 & 1496.61\end{array}$

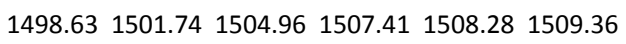
$\begin{array}{llllll}1513.43 & 1516.58 & 1518.28 & 1522.17 & 1522.79 & 1528.06\end{array}$

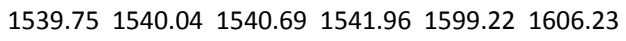

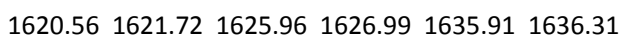
$1641.341642 .741654 .871656 .83 \quad 1662.271662 .94$ 2991.523009 .183020 .703026 .043026 .403029 .84 3035.583044 .823049 .823060 .113075 .473078 .31 $3079.613089 .743091 .73 \quad 3097.983108 .953114 .24$ 3123.313136 .363139 .603139 .863167 .473172 .87 3173.103178 .383178 .553179 .803183 .073183 .81 3187.713188 .453191 .853194 .393194 .743195 .59 3196.313197 .193199 .053199 .403199 .813204 .48 3205.013205 .863207 .223207 .603207 .663209 .70 3212.003212 .923214 .553215 .603219 .253221 .76 3241.133249 .443259 .26

\begin{tabular}{|c|c|c|c|c|c|}
\hline \multicolumn{6}{|c|}{56} \\
\hline & ======= & :====== & l====== & :====== & $===$ \\
\hline 3.90 & 6.40 & 13.16 & 16.38 & 18.902 & 27.84 \\
\hline 30.12 & 32.03 & 32.59 & 36.24 & 38.11 & 39.35 \\
\hline 44.20 & 46.74 & 49.84 & 53.55 & 54.95 & 57.53 \\
\hline 61.78 & 63.44 & 64.58 & 70.94 & 75.19 & 79.91 \\
\hline 82.26 & 89.56 & 92.62 & 99.38 & 109.28 & 114.06 \\
\hline 129.18 & 132.04 & 144.54 & 149.81 & 152.10 & 157.58 \\
\hline 168.93 & 182.90 & 184.45 & 189.12 & 197.98 & 202.70 \\
\hline
\end{tabular}

$\begin{array}{llllll}203.35 & 207.28 & 212.17 & 223.03 & 223.73 & 231.27\end{array}$ $\begin{array}{llllll}239.51 & 244.52 & 255.72 & 258.41 & 263.65 & 273.33\end{array}$ $\begin{array}{llllll}282.00 & 285.00 & 312.15 & 327.62 & 331.49 & 351.51\end{array}$ $\begin{array}{llllll}352.41 & 357.19 & 376.88 & 379.91 & 402.15 & 404.75\end{array}$ $\begin{array}{llllll}411.67 & 412.34 & 417.37 & 417.97 & 419.37 & 419.90\end{array}$ $\begin{array}{lllllll}424.90 & 436.87 & 440.19 & 442.90 & 449.07 & 452.28\end{array}$ $\begin{array}{llllll}469.86 & 487.35 & 493.50 & 499.67 & 501.88 & 517.07\end{array}$ $\begin{array}{llllll}530.48 & 587.46 & 611.06 & 613.26 & 613.86 & 614.62\end{array}$ $\begin{array}{llllll}617.11 & 618.91 & 622.16 & 630.88 & 631.81 & 633.14\end{array}$ $\begin{array}{llllll}634.56 & 635.45 & 656.85 & 659.58 & 662.40 & 679.50\end{array}$ $\begin{array}{llllll}688.08 & 693.46 & 700.65 & 712.48 & 719.14 & 720.11\end{array}$ $\begin{array}{llllll}722.81 & 727.44 & 749.73 & 751.92 & 756.03 & 757.25\end{array}$ $\begin{array}{llllll}760.65 & 773.26 & 777.27 & 794.37 & 799.81 & 811.11\end{array}$ $\begin{array}{lllllll}820.84 & 831.72 & 833.67 & 843.75 & 848.10 & 854.36\end{array}$ $\begin{array}{llllll}866.11 & 869.21 & 869.58 & 873.28 & 875.13 & 876.67\end{array}$ $\begin{array}{llllll}890.27 & 900.48 & 926.53 & 930.18 & 934.39 & 938.75\end{array}$ $\begin{array}{llllll}940.44 & 943.73 & 946.55 & 948.70 & 958.83 & 975.46\end{array}$ $\begin{array}{llllll}978.47 & 982.32 & 983.20 & 983.93 & 985.56 & 987.79\end{array}$

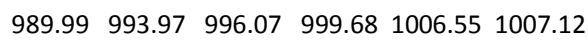
$1008.77 \quad 1009.301011 .491013 .381013 .911016 .33$

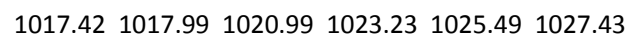
$1030.541031 .16 \quad 1031.191031 .621035 .541044 .92$ $\begin{array}{llllll}1054.44 & 1054.73 & 1054.92 & 1055.92 & 1056.13 & 1060.68\end{array}$ $\begin{array}{llllll}1062.10 & 1073.63 & 1075.86 & 1077.02 & 1089.86 & 1092.42\end{array}$ $\begin{array}{llllll}1103.05 & 1112.48 & 1115.97 & 1117.61 & 1117.63 & 1119.20\end{array}$

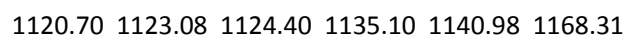
$1178.771199 .13 \quad 1200.391201 .961202 .331208 .86$ $\begin{array}{lllll}1209.94 & 1210.401212 .57 & 1214.85 & 1216.74 & 1217.72\end{array}$ $\begin{array}{llllll}1220.33 & 1223.67 & 1228.14 & 1228.69 & 1229.17 & 1231.09\end{array}$ $\begin{array}{llllll}1233.60 & 1236.90 & 1254.92 & 1258.45 & 1265.89 & 1268.52\end{array}$

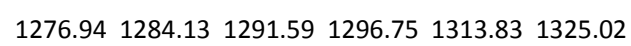
$\begin{array}{llllll}1333.10 & 1336.21 & 1336.76 & 1338.55 & 1343.61 & 1344.41\end{array}$ $\begin{array}{llllll}1351.02 & 1356.99 & 1357.49 & 1359.60 & 1362.82 & 1362.88\end{array}$

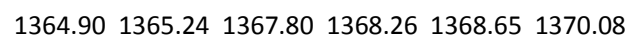
$\begin{array}{llllll}1385.73 & 1386.70 & 1392.24 & 1399.69 & 1404.70 & 1406.88\end{array}$ $\begin{array}{llllll}1417.76 & 1427.68 & 1437.08 & 1444.80 & 1459.40 & 1472.18\end{array}$ $\begin{array}{llllll}1476.02 & 1478.81 & 1485.55 & 1493.83 & 1494.93 & 1495.46\end{array}$ $\begin{array}{llllll}1495.60 & 1502.41 & 1506.18 & 1507.38 & 1509.06 & 1515.15\end{array}$ $\begin{array}{llllll}1515.39 & 1516.89 & 1517.25 & 1517.94 & 1520.52 & 1520.72\end{array}$

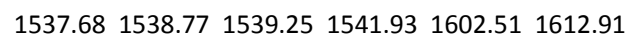
$\begin{array}{llllll}1616.14 & 1617.57 & 1624.19 & 1626.88 & 1638.17 & 1638.44\end{array}$ 
1638.641641 .001656 .971658 .141658 .811662 .13 $3018.303024 .793029 .83 \quad 3036.143042 .313044 .73$ $3051.503052 .293060 .03 \quad 3065.033079 .483080 .84$ 3081.823096 .453103 .413107 .043113 .853115 .38 3116.343118 .243135 .293156 .973161 .393166 .49 3167.863169 .263171 .093171 .683171 .963183 .02 3183.383184 .543187 .513188 .113188 .673190 .77 3191.673194 .983195 .083195 .503197 .113197 .65 3197.733202 .403203 .373205 .673208 .933209 .12 3209.293210 .223210 .343210 .843220 .393220 .47 3229.603233 .243245 .70

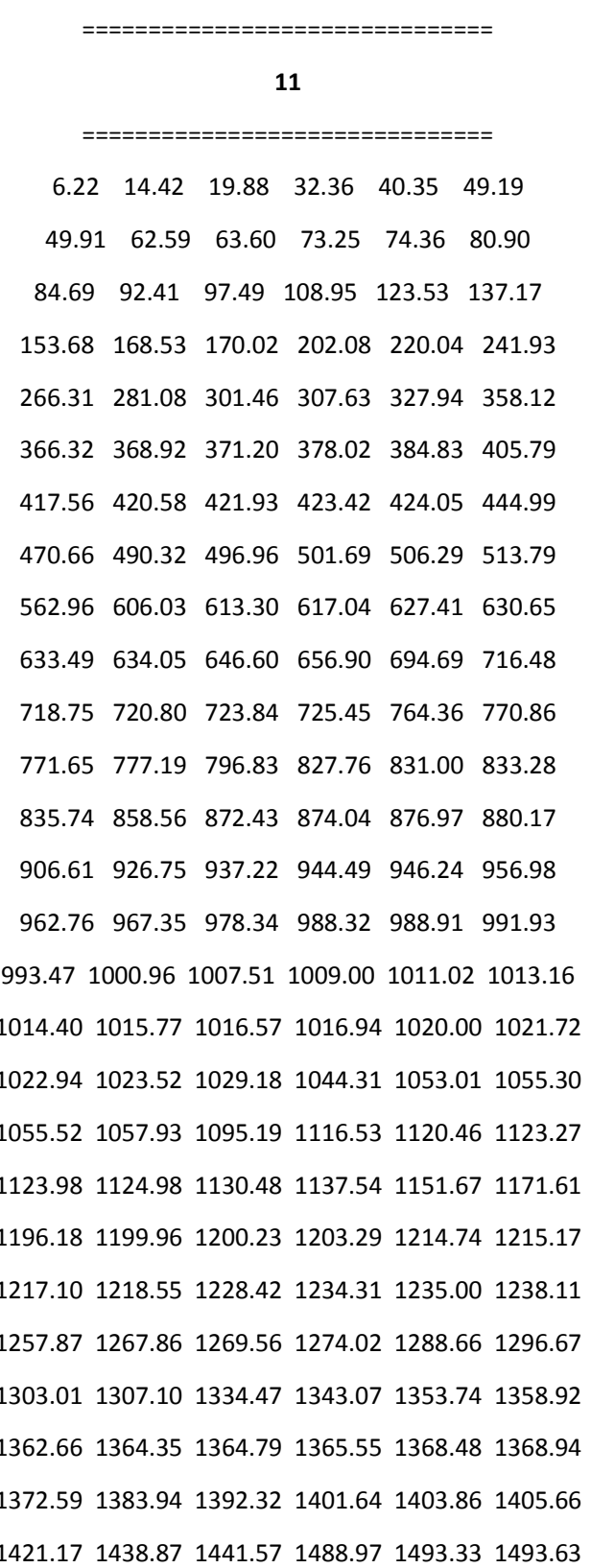

$\begin{array}{llllll}1495.48 & 1497.42 & 1512.35 & 1517.33 & 1521.02 & 1522.72\end{array}$

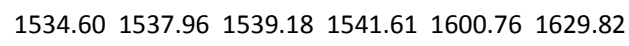

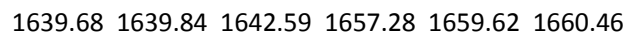
1661.892998 .423021 .063028 .913050 .613058 .84 3075.343081 .223100 .833110 .183114 .113117 .85

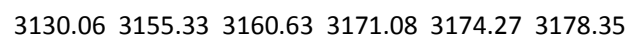
3179.623180 .243180 .923185 .873186 .423187 .41 3189.153193 .263195 .153196 .263199 .763200 .88 3205.573206 .543206 .683210 .543211 .953220 .05

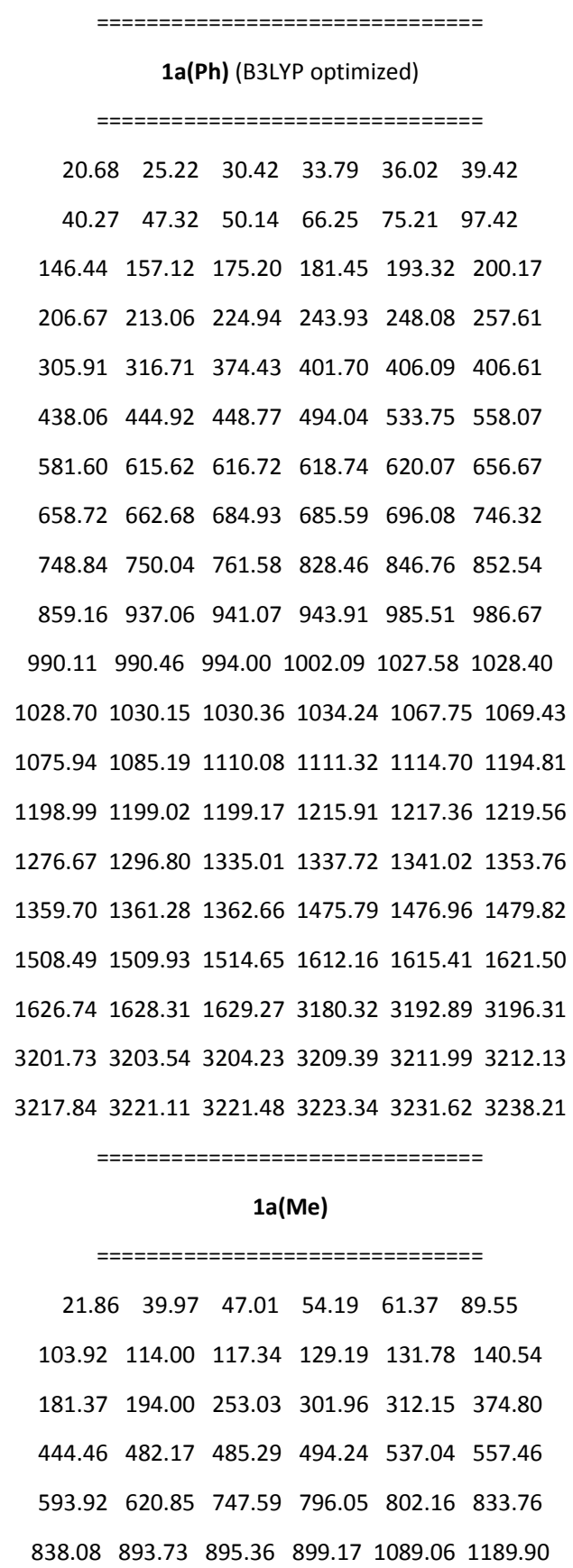




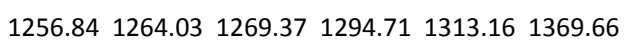
1438.771446 .201450 .341452 .741455 .911464 .20 3077.623079 .073082 .463192 .103194 .313199 .48 3199.673213 .343216 .84

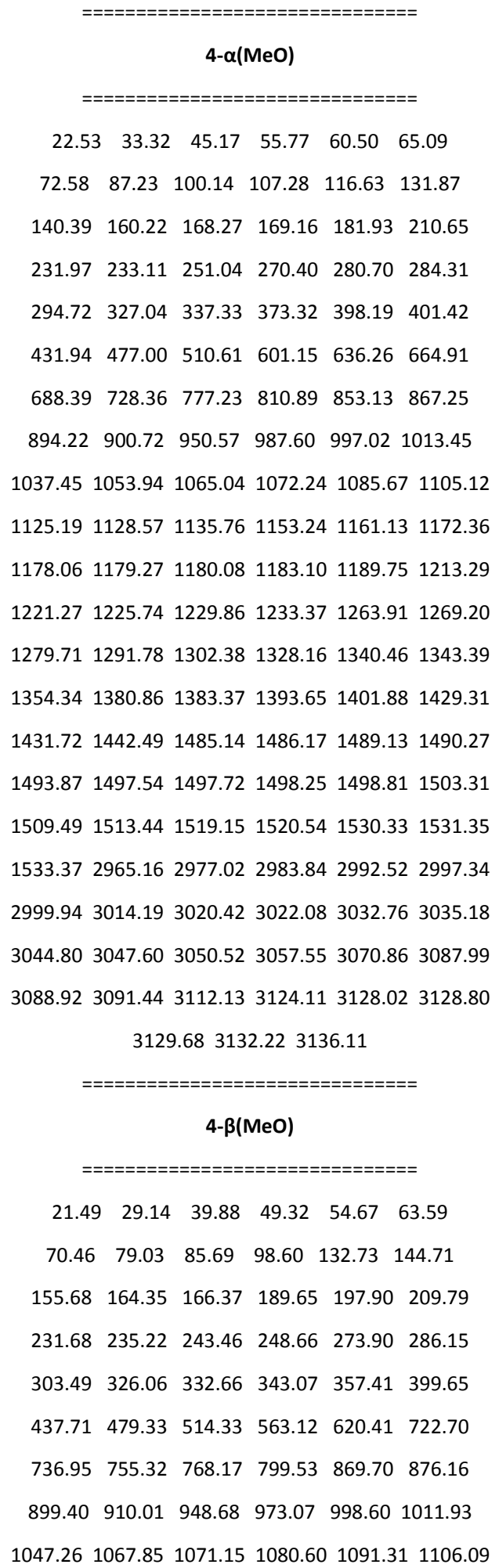

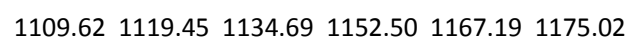

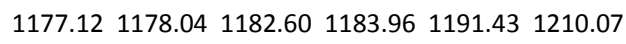

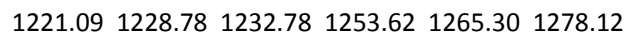

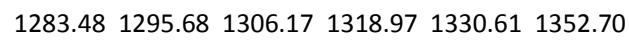

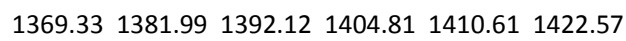
$\begin{array}{lllllll}1425.18 & 1434.54 & 1486.58 & 1487.56 & 1491.70 & 1492.73\end{array}$ 1497.451498 .691499 .131501 .871502 .811507 .50

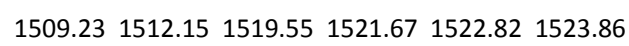
1533.522926 .772961 .922979 .652980 .392988 .63 2994.753000 .303006 .063008 .863011 .223027 .57 $3037.363038 .843040 .203056 .213071 .63 \quad 3076.77$ 3078.633079 .743109 .883113 .793126 .373126 .65 3127.973130 .573130 .81

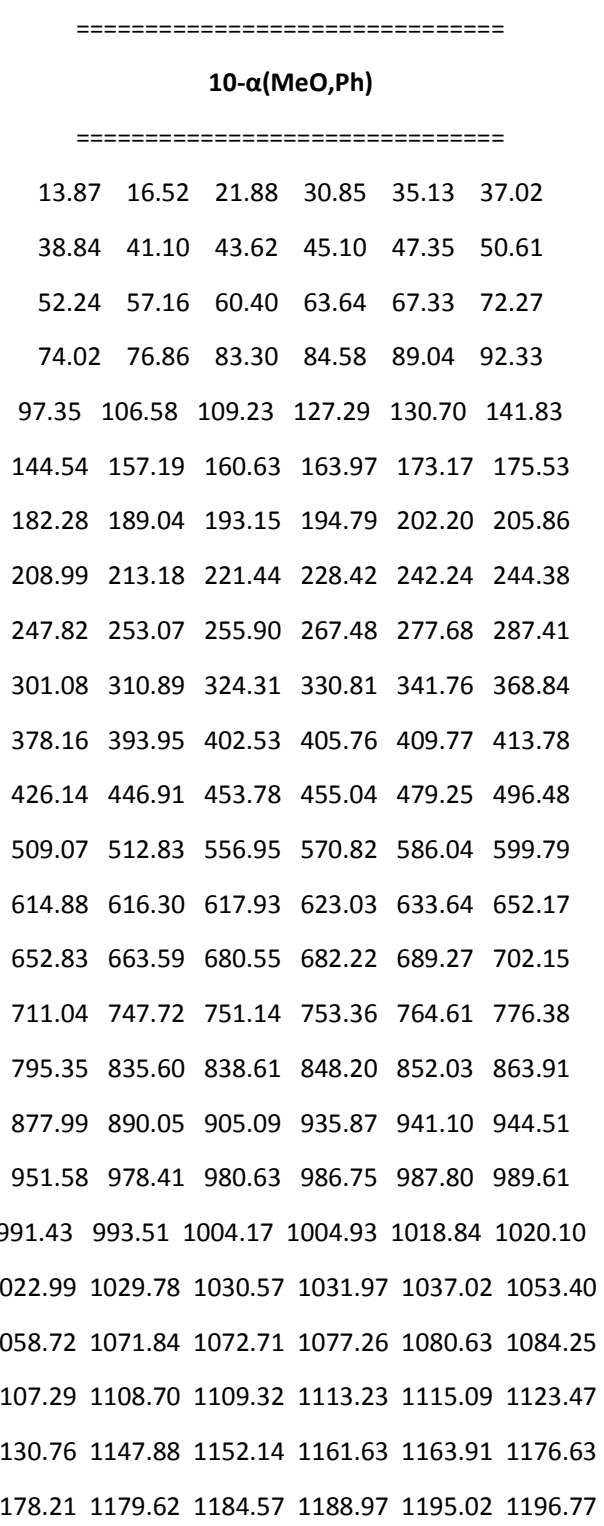


$1196.791212 .661214 .14 \quad 1217.491220 .461221 .68$ $1222.91 \quad 1225.291233 .581244 .451252 .841265 .66$

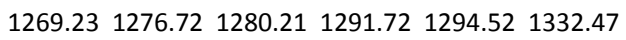

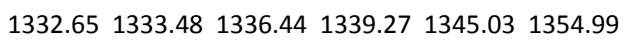

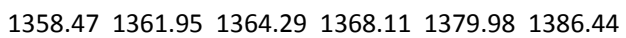
1400.181401 .401430 .101434 .191441 .541474 .41 $\begin{array}{llllll}1477.33 & 1478.53 & 1480.21 & 1486.18 & 1486.86 & 1491.35\end{array}$

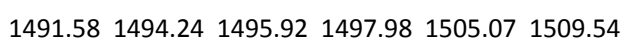

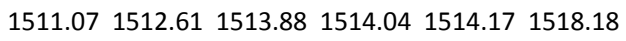
$\begin{array}{llllll}1518.44 & 1528.98 & 1530.93 & 1532.81 & 1610.82 & 1614.50\end{array}$ $\begin{array}{lllll}1625.60 & 1628.23 & 1630.30 & 1632.33 & 2987.38 \\ 2992.41\end{array}$ $2996.723003 .693008 .283012 .93 \quad 3021.693034 .17$ $3036.523042 .923055 .283057 .323064 .33 \quad 3066.55$ 3080.263090 .883106 .063113 .923116 .413128 .15 3129.993147 .973148 .863152 .593157 .183168 .17 3188.243192 .743194 .023195 .423200 .973201 .90 3204.173208 .353211 .023213 .793217 .423217 .88 3221.413234 .393238 .55

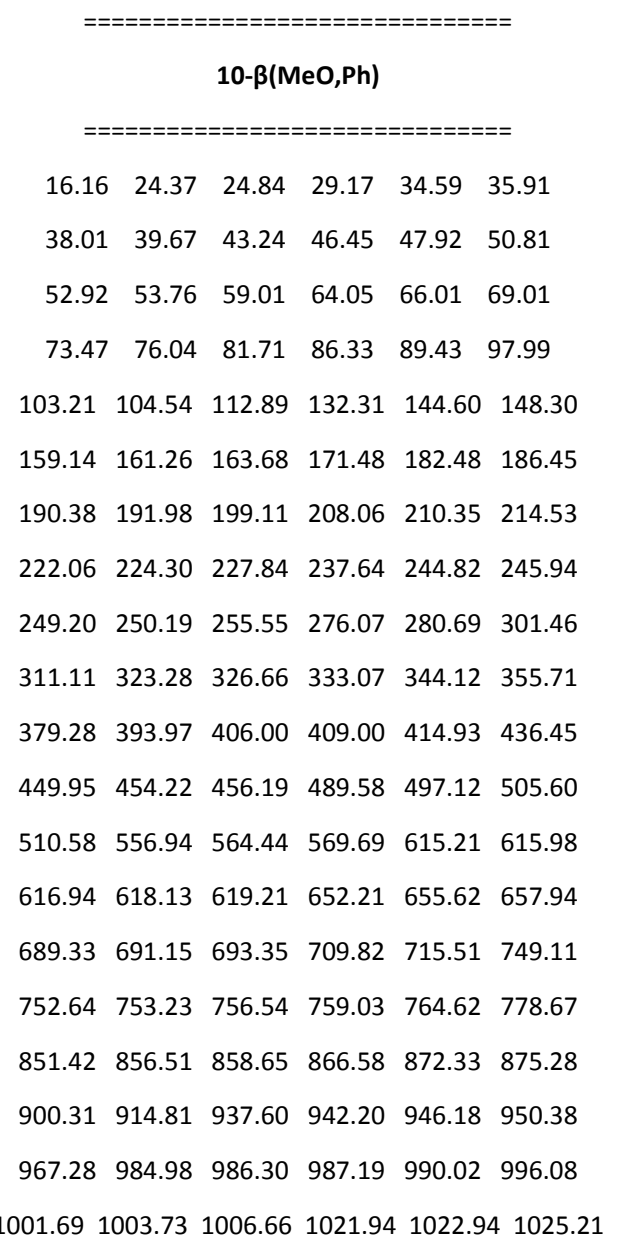

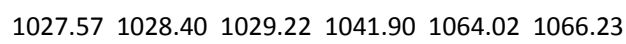

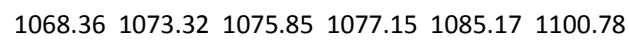
1108.411109 .241110 .631114 .031115 .941119 .55 $\begin{array}{lll}1122.34 & 1147.641160 .601168 .21 & 1172.651175 .08\end{array}$ 1178.741181 .571186 .651193 .371196 .631196 .72

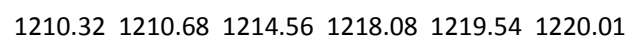

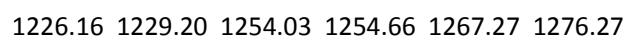

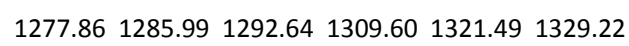
$\begin{array}{lllll}1334.01 & 1336.07 & 1337.501338 .05 & 1354.73 & 1355.64\end{array}$ $\begin{array}{llllll}1361.05 & 1363.72 & 1368.55 & 1376.50 & 1393.79 & 1401.37\end{array}$ $\begin{array}{llllll}1412.78 & 1421.96 & 1430.61 & 1434.91 & 1475.04 & 1477.34\end{array}$ $\begin{array}{llllll}1477.69 & 1486.08 & 1488.20 & 1489.54 & 1491.39 & 1493.72\end{array}$

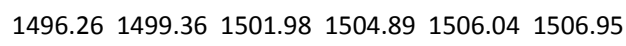
$\begin{array}{llllll}1510.24 & 1512.33 & 1513.43 & 1514.16 & 1515.40 & 1519.12\end{array}$ $\begin{array}{llllll}1520.96 & 1527.02 & 1532.25 & 1611.32 & 1616.55 & 1621.36\end{array}$ $\begin{array}{lllll}1628.88 & 1631.56 & 1632.162966 .52 & 2978.36 & 2989.69\end{array}$ 3000.563002 .373004 .283012 .013014 .943030 .71 3035.193038 .863051 .943055 .703059 .993074 .08 3079.633082 .553091 .053105 .843124 .893130 .87 3138.523138 .883146 .523151 .903157 .943187 .41 3195.653195 .733197 .093203 .453204 .723206 .40 3213.303213 .893216 .593219 .613222 .173223 .87 3225.093226 .29

\begin{tabular}{rllllll}
\multicolumn{1}{c}{$======================$} \\
\multicolumn{7}{c}{$10-\alpha(\mathbf{M e O}, \mathrm{Me})$} \\
17.36 & 19.24 & 26.57 & 32.94 & 36.48 & 40.98 \\
43.38 & 49.22 & 58.26 & 59.15 & 71.63 & 72.30 \\
74.37 & 80.80 & 85.23 & 92.95 & 97.38 & 102.89 \\
07.75 & 108.38 & 120.94 & 126.73 & 132.71 & 144.25 \\
46.91 & 151.70 & 157.78 & 162.53 & 163.91 & 172.98 \\
73.44 & 177.22 & 185.41 & 186.53 & 192.84 & 205.16 \\
10.77 & 224.22 & 227.71 & 244.87 & 245.49 & 258.74 \\
66.22 & 277.93 & 286.28 & 303.02 & 308.80 & 322.46 \\
30.79 & 341.03 & 368.69 & 372.02 & 391.60 & 404.62 \\
26.13 & 444.40 & 478.07 & 485.87 & 490.09 & 497.15 \\
10.86 & 522.26 & 559.22 & 575.42 & 583.07 & 595.93 \\
18.13 & 632.57 & 683.01 & 710.80 & 765.25 & 778.91 \\
92.21 & 810.71 & 831.58 & 839.15 & 841.34 & 861.09 \\
868.66 & 891.25 & 895.41 & 903.10 & 903.46 & 924.85 \\
2.81 & 976.70 & 985.74 & 1002.76 & 1031.84 & 1052.68
\end{tabular}


$1057.25 \quad 1075.92 \quad 1083.45 \quad 1107.87 \quad 1111.691123 .32$ $1129.201142 .911151 .331161 .83 \quad 1162.681175 .64$ $1178.101178 .131182 .63 \quad 1189.451206 .091213 .73$ 1222.011224 .991232 .951244 .211246 .841247 .50 $\begin{array}{llllll}1254.71 & 1266.02 & 1268.97 & 1280.18 & 1283.67 & 1287.32\end{array}$

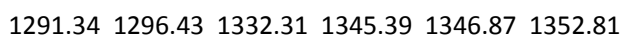

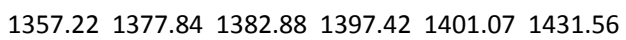
$\begin{array}{llllll}1435.52 & 1442.35 & 1449.53 & 1450.81 & 1453.95 & 1458.02\end{array}$ 1464.981482 .081483 .351486 .591487 .541491 .55 1491.941494 .121494 .621498 .001505 .801510 .40

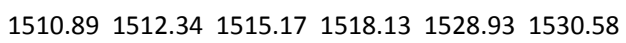
1531.662989 .292995 .603001 .653011 .473014 .19 3015.813018 .823026 .953034 .843041 .773058 .23 $3070.043071 .763072 .383073 .63 \quad 3075.353079 .80$ 3086.803090 .143106 .813109 .623121 .963131 .23 3133.873151 .803152 .723159 .883161 .703181 .80 3199.753207 .693212 .633213 .213214 .983222 .52

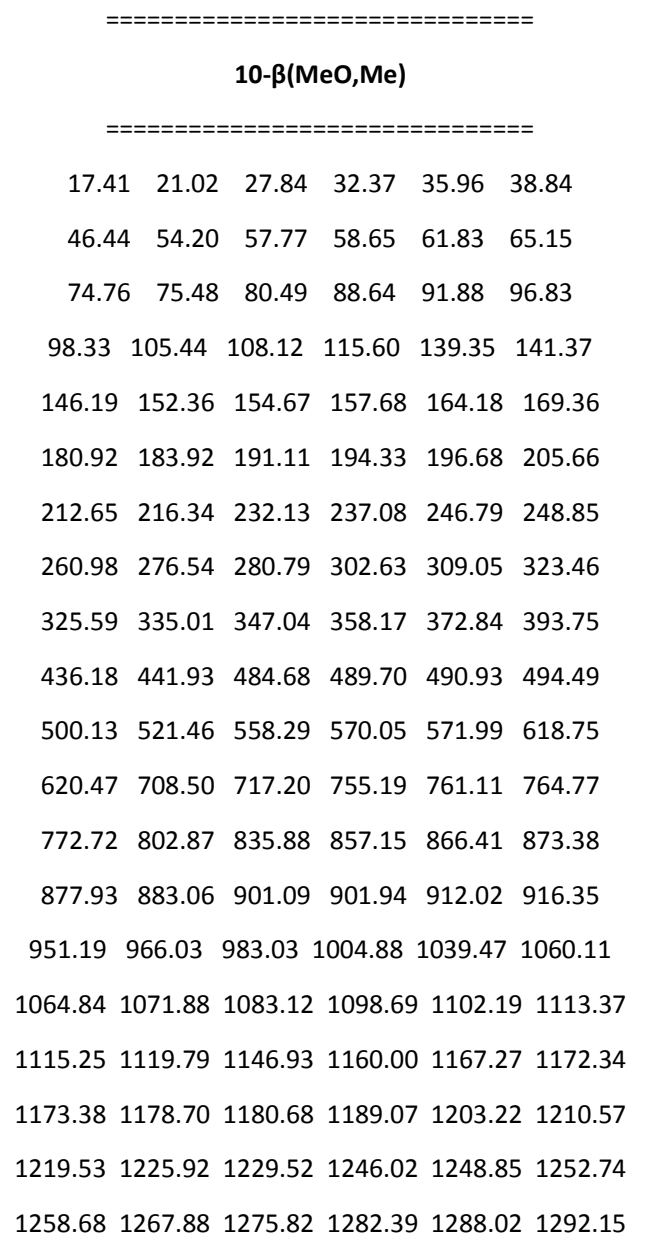

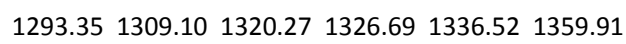
1368.241374 .441395 .811402 .091409 .721423 .20

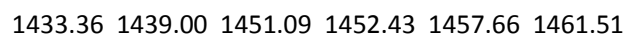

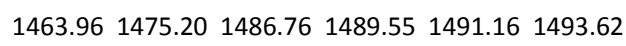

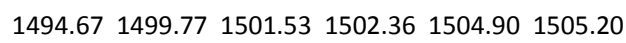
$\begin{array}{llllll}1512.19 & 1514.501515 .96 & 1518.46 & 1520.97 & 1527.27\end{array}$ $1533.782973 .93 \quad 2984.962991 .83 \quad 3008.493014 .03$ $3014.193014 .953020 .223025 .783034 .73 \quad 3036.52$

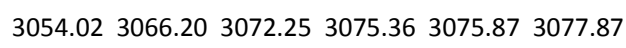
$3086.003086 .473090 .283090 .353115 .52 \quad 3126.23$ $3135.893142 .43 \quad 3147.443147 .92 \quad 3151.04 \quad 3161.27$ 3197.723198 .723205 .203210 .053221 .373235 .73 


\section{References}

(1) Boyd, T. J.; Geerts, Y.; Lee, J. K.; Fogg, D. E.; Lavoie, G. G.; Schrock, R. R.; Rubner, M. F. Macromolecules 1997, 30, 3553.

(2) Neese, F. Wiley Interdisclipinary Reviews - Computational Molecular Science 2012, 2, 73.

(3) Becke, A. D. Physical Review A 1988, 38, 3098.

(4) Vosko, S. H.; Wilk, L.; Nusair, M. Canadian Journal of Physics 1980, 58, 1200.

(5) Lee, C. T.; Yang, W. T.; Parr, R. G. Physical Review B 1988, 37, 785.

(6) Stephens, P. J.; Devlin, F. J.; Chabalowski, C. F.; Frisch, M. J. J. Phys. Chem. $1994,98,11623$.

(7) Grimme, S.; Antony, J.; Ehrlich, S.; Krieg, H. J. Chem. Phys. 2010, 132, 154104.

(8) Goerigk, L.; Grimme, S. Phys. Chem. Chem. Phys. 2011, 13, 6670.

(9) Hariharan, P. C.; Pople, J. A. Theoret. Chim. Acta 1973, 28, 213.

(10) Francl, M. M.; Pietro, W. J.; Hehre, W. J.; Binkley, J. S.; Gordon, M. S.; DeFrees, D. J.; Pople, J. A. J. Chem. Phys. 1982, 77, 3654.

(11) Wadt, W. R.; Hay, P. J. J. Chem. Phys. 1985, 82, 284.

(12) Dunning, T. H., Jr. J. Chem. Phys. 1989, 90, 1007.

(13) Woon, D. E.; Dunning, T. H. J. Chem. Phys. 1993, 98, 1358.

(14) Roy, L. E.; Hay, P. J.; Martin, R. L. J. Chem. Theory Comput. 2008, 4, 1029.

(15) Kendall, R. A.; Dunning, T. H.; Harrison, R. J. J. Chem. Phys. 1992, 96, 6796.

(16) Marten, B.; Kim, K.; Cortis, C.; Friesner, R. A.; Murphy, R. B.; Ringnalda, M. N.; Sitkoff, D.; Honig, B. J. Phys. Chem. 1996, 100, 11775.

(17) Friedrichs, M.; Zhou, R. H.; Edinger, S. R.; Friesner, R. A. J. Phys. Chem. B 1999, 103, 3057.

(18) Edinger, S. R.; Cortis, C.; Shenkin, P. S.; Friesner, R. A. J. Phys. Chem. B 1997, 101, 1190.

(19) Rashin, A. A.; Honig, B. J. Phys. Chem. 1985, 89, 5588.

(20) Vykhodtseva, L. N.; Nekrasov, L. N.; Peregudova, S. M. Vestnik Moskovskogo Universiteta Seriya 2 Khimiya 1987, 28, 452.

(21) Gajewski, J. J.; Gilbert, K. E.; McKelvie, J. In Advances in Molecular Modeling; Liotta, D., Ed.; JAI Press: Greenwich, CT, 1990; Vol. 2, p 65.

(22) Zhao, Y.; Truhlar, D. Theoretical Chemistry Accounts 2008, 120, 215. 